\title{
Polarization-Modulated Second Harmonic Generation Microscopy in Collagen
}

P. Stoller

Ph.D Thesis

September 30, 2002

U.S. Department of Energy

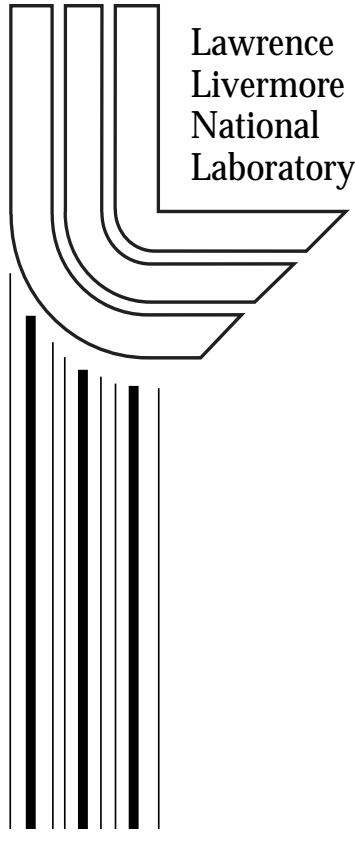




\section{DISCLAIMER}

This document was prepared as an account of work sponsored by an agency of the United States Government. Neither the United States Government nor the University of California nor any of their employees, makes any warranty, express or implied, or assumes any legal liability or responsibility for the accuracy, completeness, or usefulness of any information, apparatus, product, or process disclosed, or represents that its use would not infringe privately owned rights. Reference herein to any specific commercial product, process, or service by trade name, trademark, manufacturer, or otherwise, does not necessarily constitute or imply its endorsement, recommendation, or favoring by the United States Government or the University of California. The views and opinions of authors expressed herein do not necessarily state or reflect those of the United States Government or the University of California, and shall not be used for advertising or product endorsement purposes.

This work was performed under the auspices of the U. S. Department of Energy by the University of California, Lawrence Livermore National Laboratory under Contract No. W-7405-Eng-48.

This report has been reproduced directly from the best available copy.

Available electronically at http://www.doe.gov/bridge

Available for a processing fee to U.S. Department of Energy

and its contractors in paper from

U.S. Department of Energy

Office of Scientific and Technical Information

P.O. Box 62

Oak Ridge, TN 37831-0062

Telephone: (865) 576-8401

Facsimile: (865) 576-5728

E-mail: reports@adonis.osti.gov

Available for the sale to the public from

U.S. Department of Commerce

National Technical Information Service

5285 Port Royal Road

Springfield, VA 22161

Telephone: (800) 553-6847

Facsimile: (703) 605-6900

E-mail: orders@ntis.fedworld.gov

Online ordering: http://www.ntis.gov/ordering.htm

OR

Lawrence Livermore National Laboratory

Technical Information Department's Digital Library

http://www.llnl.gov/tid/Library.html 
Patrick C. Stoller

December 2002

Engineering-Applied Science

Polarization-modulated second harmonic generation microscopy in collagen

\begin{abstract}
$\underline{\text { Abstract }}$
Collagen is a key structural protein in the body; several pathological conditions lead to changes in collagen. Among imaging modalities that can be used in vivo, second harmonic generation (SHG) microscopy has a key advantage: it provides $\sim 1 \mu \mathrm{m}$ resolution information about collagen structure as a function of depth. A new technique-polarization-modulated SHG-is presented: it permits simultaneous measurement of collagen orientation, of a lower bound on the magnitude of the second order nonlinear susceptibility tensor, and of the ratio of the two independent elements in this tensor. It is applied to characterizing SHG in collagen and to determining effects of biologically relevant changes in collagen structure.

The magnitude of the second harmonic signal in two dimensional images varies with position even in structurally homogeneous tissue; this phenomenon is due to interference between second harmonic light generated by neighboring fibrils, which are randomly oriented parallel or anti-parallel to each other. Studies in which focal spot size was varied indicated that regions where fibrils are co-oriented are less than $\sim 1.5 \mu \mathrm{m}$ in diameter. A quartz reference was used to determine the spot size as well as a lower limit $\left(d_{x x x}>0.3 \mathrm{pm} / \mathrm{V}\right)$ for the magnitude of the second order nonlinear susceptibility. The ratio of the two independent tensor elements ranged between $d_{X Y Y} / d_{X X X}=0.60$ and 0.75 .
\end{abstract}


SHG magnitude alone was not useful for identifying structural anomalies in collagenous tissue. Instead, changes in the polarization dependence of SHG were used to analyze biologically relevant perturbations in collagen structure. Changes in polarization dependence were observed in dehydrated samples, but not in highly crosslinked samples, despite significant alterations in packing structure. Complete thermal denaturation and collagenase digestion produced samples with no detectable SHG signal.

Collagen orientation was measured in thin samples of several different tissues in transmission mode as well as at different depths (up to $200 \mu \mathrm{m}$ ) in thick samples in reflection mode; birefringence had no effect on the measurement. These studies showed that SHG microscopy was capable of detecting pathophysiological changes in collagen structure, suggesting that this technique has potential clinical applications. 


\section{BACKGROUND AND INTRODUCTION}

\section{Collagen}

Collagen is the most abundant structural protein in the human body: it accounts for approximately $12 \%$ of the body's dry weight. Collagen takes on many different forms and plays many different roles in the body; it is found in and gives structure to bone, tendon, skin, cornea, artery, and the fascia surrounding internal organs, to name just a few examples. There is a complex interaction between collagen and the resident cell populations. Many pathological conditions are associated with derangements in collagen structure and function. Techniques capable of detecting such changes rapidly and non-invasively have great potential for clinical applications.

Early research into the properties of collagen was driven by the glue manufacturing and leather tanning industries and focused mainly on the practical aspects of glue and tanning chemistry (Heidemann, 1988). Growing scientific interest in understanding the properties of important biological molecules, coupled with the development of x-ray diffraction, a technique that provided information on the scale of a nanometer, led to a new era of research into the structure of proteins and nucleic acids. Shortly after the double-helical structure of DNA was first suggested (Watson and Crick, 1953), the basic structure of the collagen molecule - a triple helix consisting of three $\alpha$ helices coiled around each other-was deduced by Ramachandran and Kartha (1954). An extensive review of research into the molecular structure of collagen is presented in Chemistry of Collagen (1967).

The discovery of the triple-helical structure of collagen opened new fields of research into the organization of collagen molecules into larger structures, into the 
chemical modification of these structures, and into the interaction of collagen with other components of the extracellular matrix and with cells. This research has demonstrated that, unlike most proteins, collagen has a half-life measured in decades, during which time it undergoes many post-translational modifications; these modifications have a profound impact not only on the structure and function of the collagen itself, but also on its interaction with cells. Some modifications, particularly those driven by stochastic forces, have been shown to play a significant role in the degenerative diseases associated with aging and diabetes (Reiser, 1998). Collagen (1988), a five-volume series, presents an authoritative overview of the state of research into collagen written by major contributors to the field.

There are more than twenty genetically distinct types of collagen; they are loosely categorized into two groups-the fibril forming collagens (types I, II, III, and V, for example) and the non-fibrillar collagens (type IV, for example). Most tissues comprise several collagen types, each serving a specific role. In skin, for instance, type IV collagen is found in the basement membrane that separates the epidermis from the dermis. Types I and III collagen fibrils are abundant in the dermis. Thin fibrils of type VII collagen anchor the dermal collagen fibrils to the type IV collagen of the basement membrane.

This work focuses on the development of a novel imaging system based on the second order nonlinear optical properties of collagen. This chapter consists of a brief overview of the structure and organization of type I collagen (the collagen type used in most of these studies), of related pathological conditions, and of the current imaging 
techniques. In subsequent chapters, the theoretical basis, experimental techniques, and applications of these techniques to the analysis of native tissue are presented. 


\section{Type I collagen}

A single type I collagen molecule has a triple-helical structure, with short, nonhelical extensions at both ends; it has a length of about $300 \mathrm{~nm}$ and a diameter of about $1.5 \mathrm{~nm}$. Collagen is produced as procollagen inside the cytoplasm of cells, primarily in specialized fibroblast cells. Procollagen has a triple-helical structure like collagen, but it has longer non-helical extensions at both ends. Procollagen undergoes extensive posttranslational modifications inside the cell, including hydroxylation of some lysine and proline residues. Each procollagen molecule is composed of two identical alpha chains and a third alpha chain with somewhat different primary structure. The alpha chains each are composed of approximately 1000 amino acids, and, except in the non-helical extensions, the amino acids are in a glycine- $X-Y$ sequence, where $X$ is often proline and $\mathrm{Y}$ is often hydroxyproline. This characteristic triplet has important implications for secondary and tertiary structure. Beck and Brodsky (1998) present a review of the current understanding of the triple-helical molecular structure of collagen.

After procollagen molecules are extruded into the extracellular matrix by fibroblasts, part of their non-helical extensions is cleaved enzymatically. For reasons not well understood, this modification allows the collagen molecules to self-assemble into small units called microfibrils. These microfibrils contain only five or six collagen molecules; the individual molecules are staggered by $67 \mathrm{~nm}$ with respect to their neighbors. Microfibrils in turn self-organize into small fibrils with diameters on the order of $15 \mathrm{~nm}$ (Figure 1.1). After cleavage of the $\mathrm{N}$-terminal non-helical extension at a second point, the small fibrils can aggregate to form larger fibrils, with diameters ranging between $15 \mathrm{~nm}$ and $500 \mathrm{~nm}$. The organized fibrils are stabilized through enzymatically- 
mediated covalent crosslinking between lysine and/or hydroxyl since residues of adjacent collagen molecules. Nimni and Harkness (1988) give a detailed explanation of collagen fibril formation, and Wess et. al. (1998) and Kadler et. al. (1996) summarize recent work that suggests collagen molecules are packed into a triclinic structure in fibrils. Graham et. al. (2000) discusses the interactions that lead to the aggregation of neighboring collagen fibrils.

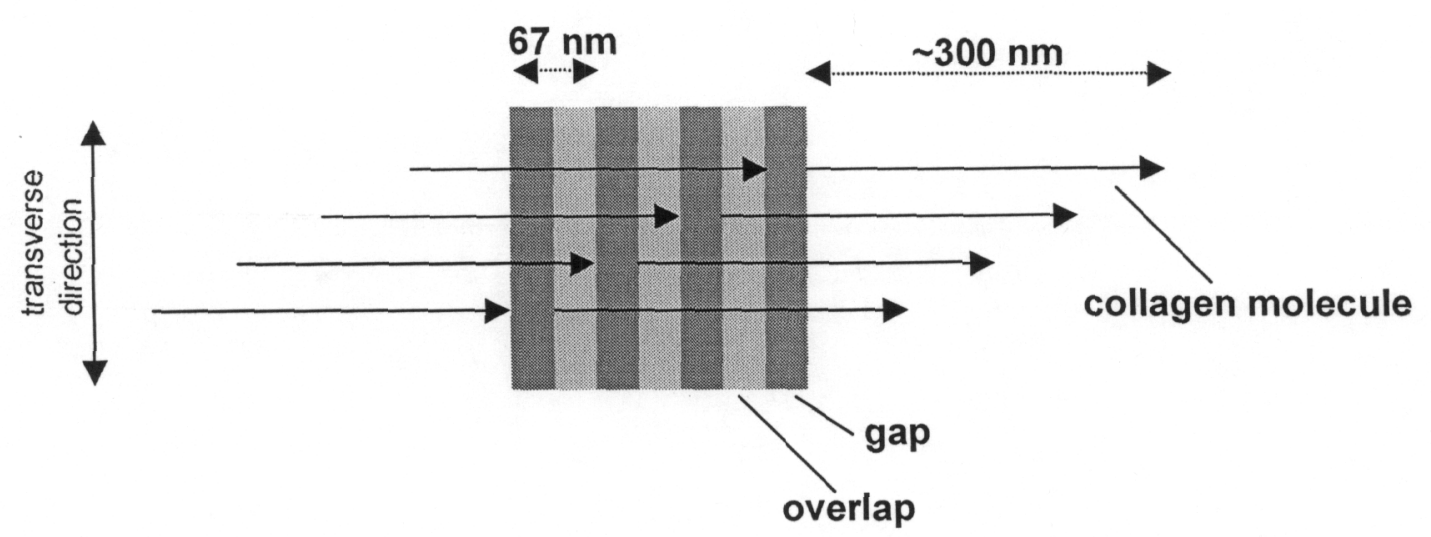

Figure 1.1 Sketch of origin of banding pattern in electron microscopy of collagen fibrils-the gap-overlap arrangement of collagen molecules. Each arrow represents an individual collagen molecule.

In some tissues, organization extends through additional levels of complexity beyond the fibrillar unit. In tendon, for example, the fascicles is the characteristic structural unit. Fascicles are structures that are centimeters in length and of order $100 \mu \mathrm{m}$ in diameter; a parallel array of collagen fibrils composes a fascicle. Individual fascicles are characterized by a crimped structure; the collagen fibrils form a zigzag pattern with adjacent segments of about $200 \mu \mathrm{m}$ in length forming an angle of 8 to 20 degrees to each other (refer, for instance, to Baer et. al., 1988). 


\section{Structural abnormalities in collagen}

Collagen provides structure and support to the body; a number of pathological conditions can interfere with the ability of collagen to carry out these functions. The pathological conditions affecting collagen can be classified into two distinct groups. The first group is characterized by the deposition of structurally abnormal collagen due to mutations in the collagen gene or in the genes of enzymes necessary for normal posttranslational processing. Examples include osteogenesis imperfecta, Ehlers-Danlos syndromes, and Marfan's syndrome. In the second group, normal collagen is synthesized and deposited, but is subsequently altered during the course of other disease processes. Examples in this second group, discussed below, include melanoma, diabetes, and osteoarthritis. These examples were selected because the ability to detect changes in collagen could potentially have clinical utility in both diagnosis and management.

About one million Americans are diagnosed with skin cancer annually; only in about $5 \%$ of these cases is the cancer a melanoma. However, melanoma, the most serious form of skin cancer, is responsible for approximately $75 \%$ of all skin cancer deaths. The key to the successful treatment of skin cancer is early detection, before melanoma cells can penetrate beneath the skin and into the lymph nodes. Friedl et. al. (1998) described the process by which neoplastic melanocytes penetrate the dermis through the activation of enzymes that destroy normal matrix structure. Thus, the ability to detect the loss of normal collagen structure in early stages could lead to earlier detection and more successful treatment of melanoma. Currently, melanoma, as well as other types of skin cancer are diagnosed by histopathological analysis of the excised lesion. For patients with dysplastic nevus syndrome, who have a large number of abnormal moles, this is not 
feasible. There have been other attempts to develop non-invasive imaging systems; a number of recent studies have had some success in applying confocal microscopy to imaging skin cancer in vivo, in an effort to eliminate the need for tissue excision (Rajadhyaksha et. al., 2001; Tannous et. al., 2002).

Diabetes refers to a condition in which the feedback mechanism that regulates the glucose level in the blood is disrupted, either through the complete cessation of insulin production or through less severe disturbances. Even when blood sugar is very closely monitored, diabetes can still lead to severe complications, ranging from kidney failure to blindness (The Diabetes Control and Complications Trial Research Group, 1993). One of the consequences of diabetes is the build-up of sugar moieties in the extracellular matrix; these sugars result in glycation of matrix proteins such as collagen. Eventually, through a complex series of reactions (the Maillard process), the sugar adducts in collagen molecules can form nonenzymatic crosslinks (Reiser, 1991; Reiser 1998). Current biochemical analysis of the degree of long-term glyco-oxidative damage in tissue requires a biopsy and therefore it is difficult to do sampling studies over extended periods of time (Contreras et. al., 1994). Thus, non-invasive techniques for observing changes in collagen structure in skin (which is related to damage in less accessible organs) could greatly improve the monitoring of diabetes patients and help in the development of new treatment modalities.

Osteoarthritis, a common and debilitating joint disease, involves the destruction of healthy cartilage which results in the loss of the surface that allows the bones of the joint to glide past each other. The severe pain that often results from this condition leads to significant restrictions in movement of affected joints. Normal cartilage is composed 
predominantly of type II collagen, proteoglycans, chondrocyte cells, and water. The type II collagen forms an arcade-like structure-with fibrils parallel to the joint near the surface and fibrils perpendicular to the surface beneath-capable of sustaining the heavy loads to which joint cartilage is subjected (Eyre, 2001). In osteoarthritic cartilage, this structure becomes progressively more disorganized and is eventually destroyed. Chondrocytes, the resident cells in cartilage, are unable to synthesize type II collagen that is integrated with the existing matrix. Disorganized type I collagen fibrils (fibrocartilage) are sometimes produced in response to this damage, but this reparative tissue lacks the structure necessary to sustain the high forces found within joints and rapidly degrades (Hunziker, 1999). Imaging collagen organization is particularly important for monitoring cartilage in tissue engineering studies aimed at enhancing repair mechanisms (Breinan et. al., 2001). Current techniques for imaging cartilage damage-visible light microscopy using an arthoscope or ultrasound imaging-are limited in their resolution or in their ability to detect small regions of disorganized tissue, especially beneath the articular surface.

These examples-melanoma, diabetes, and osteoarthritis-exemplify several major groups of pathophysiological conditions associated with modifications in collagen structure. Techniques for imaging changes in collagen can play an important role in diagnosing these and similar diseases and in understanding the fundamental biological processes that produce them. 


\section{Collagen imaging techniques}

Collagen has been imaged using a number of different techniques; they include conventional light microscopy (typically using histological stains to highlight the collagen), polarized-light microscopy, electron microscopy, and x-ray diffraction. More recently, atomic force microscopy, magnetic resonance imaging (MRI), ultrasound imaging, confocal microscopy, and optical coherence tomography (OCT) have also been used. Some of these techniques-x-ray diffraction, atomic force microscopy, and electron microscopy - can only be applied to samples in vitro; other techniques can, to a limited degree, be used in vivo. These imaging techniques given above and their application to the imaging of collagen are briefly summarized below.

Electron microscopy has allowed visualization of collagen at a resolution of a few nanometers in thin samples in transmission mode and at somewhat lower resolution in thicker samples in reflection (or "scanning") mode. Collagen samples used for electron microscopy typically require extensive preparation-dehydration, fixation in glutaraldehyde or formaldehyde, mounting in paraffin or other media, and staining with high atomic number dyes such as phosphotungstic acid or uranyl acetate-that can significantly alter the structure of the tissue. Nevertheless, a number of important discoveries about collagen stem from electron microscopy studies; these include the 67 nm quarter-stagger arrangement (also referred to as "gap-overlap" pattern) of collagen molecules in fibrils (refer, for instance, to Parry and Craig, 1988). Electron microscopy also provides information about the orientation of collagen fibrils with respect to the $\mathrm{C}$ and N-terminals. Superposed on the light and dark regions produced by the $67 \mathrm{~nm}$ quarter-stagger arrangement is an asymmetric banding pattern that can be used to 
determine orientation (Chapman, 1990). Electron microscopy is capable of identifying changes in fibrillar diameter (as occurs in degenerating cartilage) and of identifying collagen fibrils with irregular (non-circular) profiles in cross-section (Eyden et. al., 2001 presents an extensive review). The key disadvantages of electron microscopy are that it requires extensive preparation of the samples that can potentially introduce artifacts into images and that it cannot be used for in vivo studies.

Another technique that provides structural information at high resolution-X-ray diffraction-can be performed on native collagen samples. High angle X-ray diffraction provides information on short length scales--the structure of the collagen molecule or the packing arrangement of molecules in ordered fibrils. Low and medium angle X-ray diffraction gives information about fibril structure - such as the $67 \mathrm{~nm}$ gap-overlap pattern-and the organization of fibrils in tissue. Brodsky et. al. (1988) presents a review of the application of X-ray diffraction to studying collagen. X-ray diffraction has been used, for instance, to observe an increase in the diameter of glycated collagen fibrils Tanaka et. al., 1988). In addition, X-ray diffraction has been used to conclude that rat-tail tendon collagen is packed into fibrils in a triclinic structure (Wess et. al., 1998). Although X-ray diffraction can be performed in hydrated tissue samples without any further treatment, it cannot be performed in vivo because of the high intensity X-ray beams used. X-ray diffraction is limited by the presence of a degree of disorder greater than that found in crystals, even in highly organized collagen structures such as rat-tail tendon.

MRI has recently been applied to imaging collagen. Although MRI can be performed in vivo, it does not provide the same resolution as electron microscopy, or 
even conventional light microscopy. Conventional (clinically available) MRI can be used to detect large defects in collagen structure, as occur in degenerated cartilage; however, changes on the scale of several micrometers are not detectable. Microscopic MRI can be used to obtain images with resolutions of $\sim 10 \mu \mathrm{m}$. For example, Xia et. al. (2001) recently demonstrated that MRI microscopy can be employed to image the degree of collagen organization in excised articular cartilage samples. However, this technique has not been demonstrated in vivo.

Ultrasound imaging can also be applied to the study of collagen organization in tissue (Foster et. al., 2000). A key advantage is that it can be used for in vivo imaging. A number of studies (Sanghiv et. al., 1990; Kim et. al., 1995; Saied et. al., 1997) have imaged cartilage using ultrasound microscopy (with transverse resolution of order $50 \mu \mathrm{m}$ to a depth of $\sim 1 \mathrm{~cm}$ ). For instance, Töyräs (1999) demonstrated that high frequency ultrasound can be used to study articular cartilage subjected to enzymatic degradation. Ultrasound imaging is also potentially useful for studying collagen organization, but current techniques have limited resolution.

Optical microscopy provides high resolution $(\sim 1 \mu \mathrm{m})$ and, in some cases, the capability to image tissue in vivo. Conventional light microscopy of histologically stained samples (using stains specific to collagen) has many applications in pathology. However, this technique is limited by the necessity of sectioning and staining samples. Polarization microscopy, while still requiring thin sections, provides information about collagen orientation and birefringence without requiring special stains. Polarization microscopy is also fairly specific to collagen, because most other tissue components lack the birefringence of collagen. Both conventional light microscopy and polarization 
microscopy cannot be used to image thick tissues. Confocal microscopy on the other hand, allows imaging of thicker samples through a depth of a few hundred microns. In confocal microscopy, the laser beam is tightly focused onto the sample by an illuminating objective that also serves to collect back-scattered photons. Photons from near the focus pass through a pinhole, while other photons are rejected-allowing imaging with depth resolution $\sim 1 \mu \mathrm{m}$. Cullander (1998) and Masters et. al. (1997) discuss the application of confocal microscopy to imaging tissue in vivo.

One of the most promising optical techniques for imaging collagen disease in vivo is polarization sensitive optical coherence tomography (PSOCT). In conventional OCT, a beam of short coherence length $(\sim 10 \mu \mathrm{m})$ light is split; one component propagates through the sample (up to depths of a few millimeters) while the reference component propagates through a known distance in a medium with known index of refraction. When the two beams interfere, only light back-scattered from the sample from a given depth interferes constructively with the reference beam, thus providing depth resolution comparable to the coherence length. Using waveplates and polarizers, OCT sensitive to the polarization of the light can be performed. The polarization dependence of the signal for a given depth does not provide local information; it represents the polarizing properties of the tissue over the entire path the light travels. Nevertheless, because of the strong birefringence of collagen, PSOCT can be used to image collagen organization in tissue. Several studies have demonstrated that PSOCT can be used to image the depth of thermal damage to collagen (de Boer et. al., 1998; Schoenenberger et. al., 1998); heating collagen leads to a substantial reduction in its linear birefringence (Maitland and Walsh, 1997). 
In summary, while there are many different techniques for imaging collagen, none of the techniques that allow in vivo imaging at high resolution $(\sim 1 \mu \mathrm{m})$ can provide direct information about fibrillar orientation in tissue or about collagen organization at submicron scales, as a function of depth. SHG microscopy has these advantages. In the following section, pertinent studies of SHG in collagen are reviewed. 


\section{Second harmonic generation}

Optical second harmonic generation is a process in which light propagating through a material at the fundamental frequency is converted into light at double the frequency, without the absorption and reemission of photons. In the presence of an external electric field, materials are electrically polarized. If the electric field is sufficiently weak, the induced polarization is simply proportional to the strength of the field; the constant of proportionality is called the linear susceptibility of the material. At higher field strengths (such as those present in an intense laser beam), terms of higher order in the electric field begin to play a role. In the dipole approximation,

$$
\vec{P}=\varepsilon_{o}\left(\vec{\chi}^{(1)} \vec{E}+\vec{\chi}^{(2)} \vec{E} \vec{E}+\vec{\chi}^{(3)} \vec{E} \vec{E} \vec{E}+\ldots\right)
$$

Here $\varepsilon_{o}$ is the permitivity of free space, $\vec{\chi}^{(n)}$ is the nth order susceptibility tensor of the material, and $\vec{E}$ is the electric field. For certain crystal classes-those that have inversion symmetry - the even order susceptibility tensors are identically zero. Since a non-zero second order nonlinear susceptibility is required for SHG, it can only occur in materials that lack inversion symmetry. It is also important to note that the phase of the second harmonic light with respect to the first harmonic light depends on the orientation of the dipole generating it. Two oppositely oriented dipoles separated by a distance much smaller than the wavelength will generate second harmonic light that interferes destructively. Thus, to allow SHG a material must be organized on the scale of the wavelength of the light. 
Optical SHG was first observed by Franken et. al. (1961) using a pulsed ruby laser and a quartz crystal, shortly after the invention of the optical laser (Maiman, 1960). Boyd et. al. (1965) developed theory for SHG in crystals by laser beams in the plane wave limit; Kleinman et. al. (1966) extended this work to SHG by focused Gaussian beams. These studies and others that followed them have led to a good understanding of frequency doubling in crystals; the subject is treated extensively in several recent textbooks on nonlinear optics (Shen, 1984; Boyd, 1991; Guang and Song, 1999).

Although SHG was used initially to develop sources of coherent, collimated light at wavelengths other than those available from existing lasers, Hellwarth and Christensen (1974) demonstrated that it could also be used as an imaging tool. They showed that SHG microscopy provided information unavailable from conventional optical techniques, such as polarized light microscopy. The organization of a material has a significant effect on the second order nonlinear susceptibility; for example, Verbiest et. al. (1998) showed that supramolecular chirality, even on a scale smaller than the wavelength, significantly increased the second order nonlinear susceptibility of Langmuir-Blodgett films of nonracemic chiral helicenes. Because SHG is a coherent process, it is also sensitive to the phase difference between the first and second harmonic light-if the two waves are 180 degrees out of phase, the second harmonic light is converted back into first harmonic light. The orientation of the individual dipoles with respect to each other and the dispersion of the material both play an important role. Thus, it is clear that there are a number of material properties to which SHG is uniquely sensitive.

Many biological tissues are composed of macromolecules, such as collagen, with the properties necessary for SHG: the absence of inversion symmetry and organization on 
the scale of the wavelength of light. Early studies of SHG in biological samples were limited by the fact that the high intensity necessary (using Q-switched, nanosecond pulses from Nd:YAG and ruby lasers) to produce a detectable signal was close to the damage threshold of the samples. The beam also could not be focused tightly on the sample, limiting most of the studies to bulk tissue analysis. However, despite these limitations, several studies successfully measured SHG in a variety of different biological materials. The first study - using crystals of amino acids - was reported by Rieckhoff and Peticolas in 1965. Delfino (1979) built upon this earlier work and studied SHG in crystals of amino acids, dipeptides, and tripeptides, as well as in several larger proteins and viruses.

The first report of SHG in biological tissue was made by Vasilenko et. al. (1965). These investigators noted that intense infrared laser light of different wavelengths, when viewed by the naked eye, was observed as light of a frequency corresponding to the second harmonic; they suggested that SHG occurring in some part of the eye might be responsible. Fine and Hansen (1971) made the first careful measurements of SHG in collagenous tissue from animals (cornea, sclera, tendon, and skin); they used a monochromator to confirm the detected light was at the second harmonic wavelength. Roth and Freund $(1979,1981,1982)$ performed a careful study of SHG in rat-tail tendon collagen; they measured SHG as a function of the linear polarization of the laser light and developed a model based on the cylindrical symmetry of collagen fibrils. They noted that measurements made by earlier researchers of the polar component of the piezoelectric tensor in collagen, while small, were non-zero (Fukada, 1964)-and thus indicated that long-range polar order might exist. Freund and Deutsch (1986a) and Freund et. al. (1986b) used SHG microscopy to try to determine if such a "macroscopic, polar 
structure" could be observed in the arrangement of collagen fibrils in rat-tail tendon. Electron microscopy studies of collagen fibril polarity, however, suggested that individual collagen fibrils were oriented parallel or anti-parallel to their neighbors randomly. The SHG microscopy studies (Freund and Deutsch, 1986a and Freund et. al., 1986b) indicated that large regions of the rat-tail tendon samples imaged had fibrils oriented with random polarity with respect to their neighbors, but there were also areas of parallel orientation. Using crossed-beam sum frequency generation, they were able to further determine that regions of oriented collagen fibrils were less than $\sim 5 \mu \mathrm{m}$ in diameter (Freund et. al., 1986b).

Additional studies of SHG in biological tissue using nanosecond pulse or picosecond pulse Nd:YAG lasers in collagen have been performed more recently. Georgiou et. al. (2000) measured SHG in purified type I collagen over a range of laser wavelengths between $766 \mathrm{~nm}$ and $1064 \mathrm{~nm}$. Theodossiou et. al. (2002) studied the effect of heat on the second harmonic signal in purified type I collagen. They found that the second harmonic signal dropped completely after heating the samples to $\sim 65$ degrees $\mathrm{C}$ for $60 \mathrm{~min}$. SHG was also observed in human cornea (Theodossiou et. al., 2001), in fish scales (Bailey et. al., 1995), and in dentin (Altshuler et. al., 1995). Hovanessian and Lalayan (1996) compared SHG in a variety of tissues, including dermis, muscle, tendon, fascia, cartilage, aorta, and bone; they obtained the strongest signals in collagen-rich tissues such as bone, tendon, and cartilage.

The problem of tissue damage prevented tight focusing of the laser onto the sample in the studies discussed above; for instance, scanning SHG microscopy at low resolution $(\sim 50 \mu \mathrm{m})$ required compressing even samples with very weak absorption-rat- 
tail tendon - to one tenth of their original thickness (Freund et. al., 1986b). More than twenty years ago, Gannaway and Sheppard (1978) developed the first scanning SHG microscope, in which the laser beam was focused to a spot on the samples (KDP and lithium niobate) and the sample was raster-scanned to obtain an image. However, it was only the advent of the mode-locked Ti:Sapphire laser with $\sim 100 \mathrm{fs}$ pulses (see, for instance, Koechner, 1999) that allowed high resolution nonlinear optical microscopy of biological tissues without resultant damage to biological samples. Ultra-short pulse lasers permitted the use of higher light intensities necessary for SHG without requiring the high energy per pulse that led to tissue damage: A $100 \mathrm{fs}$ ultra-short pulse has a factor $10^{6}$ less energy than a $100 \mathrm{~ns}$ pulse with the same peak intensity. In addition, for pulses longer than $\sim 10 \mathrm{ps}$ in duration (where the balance between laser energy deposition and heat diffusion out of the focal volume occur on comparable time scales), the damage threshold intensity in tissue scales approximately as $\frac{1}{\sqrt{\tau}}$, where $\tau$ is the pulse duration (Wood, 1986). The damage threshold intensity continues to increase with shorter pulse width for pulses $<10$ ps in duration, though less rapidly (Stuart et. al., 1996).

Several recent reviews summarize progress made in the field of nonlinear optical microscopy and discuss the advantages of using ultra-short pulse lasers (König, 2000; Squier and Müller, 2001; Williams et. al., 2001). Initially, research in biological samples using ultra-short pulse lasers for nonlinear microscopy focused on two photon fluorescence (a process in which two photons are absorbed simultaneously and a single photon is emitted). The key advantage of two photon fluorescence, a process which depends on the square of the intensity, over single photon fluorescence is that it only occurs in the focus of the laser beam. This results in high axial resolution without 
requiring the use of a confocal pinhole. More recently, investigators have also begun to apply SHG using ultra-short pulse lasers to image biological samples. Some studies used SHG to image excised biological tissues; others imaged biological membranes and cells.

SHG in tissue using ultra-short pulse lasers $(\tau \sim 100 \mathrm{fs})$ was first reported by Guo et. al. (1996) in chicken skin. Second harmonic light was generated at single points on the sample, using a fairly large spot size. In a similar study, Kim et. al. (1999) used an amplified ultra-short pulse laser to obtain SHG in various tissues including porcine bone, porcine muscle, chicken tendon, chicken cartilage, chicken skin, and human tooth dentin. Kim et. al. (2000) presented a preliminary study of the effect of enzymatic cleavage, glycation, and thermal denaturation in tendon samples. These studies demonstrated that ultra-short pulse lasers could be used to generate detectable second harmonic light in biological tissue without damaging the samples. In these studies, however, SHG was only used for bulk measurement in tissue samples.

Gauderon et. al. (1998) presented three-dimensional SHG microscopy at high resolution $(\sim 1 \mu \mathrm{m})$ using Ti:Sapphire ultra-short pulse lasers in lithium niobate crystals. The first application of ultra-short pulse lasers to SHG microscopy in biological tissue was reported by Guo et. al. (1997). They used a mode-locked dye laser system to output $100 \mathrm{fs}$ pulses with a repetition rate of $6.5 \mathrm{kHz}$ and at a wavelength of $625 \mathrm{~nm}$. The chicken skin and muscle samples they imaged were mounted on a translation stage and were scanned through a laser beam focused using a microscope objective (with an axial resolution of $\sim 10 \mu \mathrm{m}$ and a transverse resolution of $\sim 1 \mu \mathrm{m}$ ). Because of the weak signal obtained, lock-in amplification was used and image acquisition required several hours. In a more recent study, using a Ti:Sapphire laser $(800 \mathrm{~nm})$ and a sample scanning 
microscope with $5 \mu \mathrm{m}$ transverse and $40 \mu \mathrm{m}$ axial resolution $(\sim 30$ minute image acquisition time), Guo et. al. (1999) obtained SHG images of tumors in hamster cheek pouch tissue. They were able to determine the depth of penetration of the tumor at different clinical stages of tumor progression.

Gauderon et. al. (2001a, 2001b) modified an inverted, laser scanning microscope to allow SHG detection--this system provided much more rapid image acquisition than was possible in previous studies. SHG and two photon fluorescence images were obtained simultaneously using an ultra-short pulse laser; image acquisition times of $\sim 1 \mathrm{~s}$ at resolutions of $\sim 0.5 \mu \mathrm{m}$ were possible. SHG signal was collected in transmission mode and two photon fluorescence data was collected in reflection mode. SHG images of herring sperm DNA and fruit fly chromosomes were obtained. Using a similar system (a converted upright microscope, beam scanning system, and a Ti:Sapphire oscillator) Campagnola et. al. (2002) studied SHG and two photon fluorescence in mouse ear tissue, mouse muscle tissue, and in the tubulin structures of $C$. elegans embryos. They compared the SHG and two photon fluorescence signals and they stressed that SHG is a coherent process. In tubulin, they observed that parts of the tubulin structure had a two photon fluorescence signal but no SHG signal, potentially due to the parallel or antiparallel orientation of tubulin molecules. Using beam-scanning microscopy and reflection-mode detection, Zoumi et. al. (2002) observed SHG and two photon fluorescence in vivo, using a tissue model based on a collagen gel. They studied SHG and TPEF at a number of different wavelengths, noting that collagen autofluorescence is undetectable at wavelengths of $800 \mathrm{~nm}$ and longer. 
Several studies focused on SHG in biological samples other than tissue; they measured SHG in artificial lipid bilayer membranes and in cell membranes. A number of these studies (Moreaux et. al., 2000; Moreaux et. al., 2001; Campagnola et. al., 1999; Campagnola et. al., 2000; Campagnola et. al., 2001) sought to use SHG as a measure of membrane potential by using potential-sensitive membrane staining dyes. A key advantage of this technique over fluorescence imaging is that SHG does not occur in random media such as the interior or exterior of lipid bilayers and cells; SHG is only observed in those molecules that are co-oriented in the cell membrane (Moreaux et. al., 2001, Campagnola et. al., 1999). These studies differ significantly from SHG imaging of tissue because the region of the sample that generates the signal is typically much smaller than the focal spot size (the lipid bilayers and cell membranes are tens of nanometers in thickness). 


\section{Motivation and goals}

SHG microscopy, a new technique for imaging collagen, combines a number of advantages that are not available using currently available techniques such as electron microscopy, x-ray diffraction, and optical coherence tomography: SHG microscopy in tissue is specific to collagen, provides high resolution $(\sim 1 \mu \mathrm{m})$, can potentially be used in vivo, and is sensitive to the structure and organization of collagen fibrils. While previous research has clearly demonstrated the feasibility of using SHG microscopy to image collagen in biological tissues, no study has examined in detail the relationship between structural modifications in collagen and the second order nonlinear susceptibility.

In this work, high-resolution SHG microscopy using ultra-short pulse lasers is combined with polarization dependent measurements. Thin sections of highly organized rat-tail tendon are used to eliminate linear optical effects that could interfere with measurement of the second order nonlinear susceptibility in thicker tissues. In order to avoid repeated measurements of a sample region using different polarization angles of the input beam, a polarization modulation technique that allows rapid measurement of the polarization dependence of the SHG signal was developed. This technique can be used to simultaneously measure SHG signal strength, collagen fibril orientation, and a parameter related to the ratio of the two independent elements of the second order nonlinear susceptibility tensor. By using a quartz plate to calibrate the SHG detection system, the SHG signal strength in collagen can be used to calculate a lower limit on the magnitude of the second order nonlinear susceptibility. This technique is applied to biological samples in order to determine to what extent SHG microscopy can detect biologically relevant changes in collagen structure and organization. 


\section{References}

G. B. Altshuler, N. R. Belashenkov, G. A. Martsinovski, and A. A. Solounin. 1995. Nonlinear transmission and second-harmonic generation in dentin in the field of ultrashort ND-laser pulses. In Advanced Laser Dentistry. G. B. Altshuler, R. J. Blankenau, and H. A. Wigdor, editors. Proc. SPIE 1984:6-10.

E. Baer, J. J. Cassidy, and A. Hiltner. 1988. Hierarchical structure of collagen and its relationship to the physical properties of tendon. In Collagen: Biochemistry and Biomechanics. Volume 2. Marcel E. Nimni, editor. CRC Press, Boca Raton. 177199.

R. T. Bailey, F. R. Cruickshank, D. Pugh, J. N. Sherwood, and S. Wilkie. 1995. Nonlinear optical behaviour of fish scales. Ferroelectrics. 1995:293-299.

K. Beck and B. Brodsky. 1998. Supercoiled protein motifs: the collagen triple-helix and the $\alpha$-helical coiled coil. Journal of Structural Biology. 122:17-29.

R.W. Boyd. 1992. Nonlinear Optics. Academic Press, San Diego.

G. D. Boyd, A. Ashkin, J. M. Dziedzic, and D. A. Kleinman. 1965. Second-harmonic generation of light with double refraction. Physical Review. 137:A1305-A1320.

H. A. Breinan, T. Minas, H-P. Hsu, S. Nehrer, S. Shortkroff, and M. Spector. 2001. Autologous chondrocyte implantation in a canine model: change in composition of reparative tissue with time. Journal of Orthopaedic Research. 19:482-492.

B. Brodsky, S. Tanaka, and E. F. Eikenberry. 1988. X-ray diffraction as a tool for studying collagen structure. In Collagen: Biochemistry. Volume 1. Marcel E. Nimni, editor. CRC Press, Boca Raton. 95-111. 
P. J. Campagnola, M. Wei, A. Lewis, and L. M. Loew. 1999. High-resolution nonlinear optical imaging of live cells by second harmonic generation. Biophysical Journal. $77: 3341-3349$.

P. J. Campagnola, H. A. Clark, W. A. Mohler, A. Lewis, and L. M. Loew. 2001. Secondharmonic imaging microscopy of living cells. Journal of Biomedical Optics. 6:277-286.

P. J. Campagnola, A. C. Millard, M. Terasaki, P. E. Hoppe, C. J. Malone, and W. A. Mohler. 2002. Three-dimensional high-resolution second-harmonic generation imaging of endogenous structural proteins in biological tissues. Biophysical Journal. 81:493-508.

J. A. Chapman, M. Tzaphlidou, K. M. Meek, and K. E. Kadler. 1990. The collagen fibril-a model system for studying the staining and fixation of a protein. Electron Microscopy Review. 3:143-182.

I. Contreras, K. M. Reiser, N. Martinez, E. Giansante, T. Lopez, N. Suarez, S. Postalian, M. Molina, F. Gonzales-Mujica, M. R. Sanchez et. al. 1994. Pentosidine content in skin collagen from type II diabetic patients. Biochemical Society Transactions. 22:13S.

C. Cullander. 1998. Light microscopy of living tissue: the state and future of the art. Journal of Investigative Dermatology Symposium Proceedings. 3:166-171.

J. F. de Boer, S. M. Srinivas, A. Malekafzali, Z. Chen, and J. S. Nelson. 1998. Imaging thermally damaged tissue by polarization sensitive optical coherence tomography. Optics Express. 3:212-218. 
K. J. Busam, K. Hester, C. Charles, D. L. Sachs, C. R. Antonescu, S. Gonzalez, and A. C. Halpern. 2001. Detection of clinically amelanotic malignant melanoma and assessment of its margins by in vivo confocal scanning laser microscopy. Archives of Dermatology. 137: 923-929.

M. Delfino. 1979. A comprehensive optical second harmonic generation study of the non-centrosymmetric character of biological structures. J. Biol. Phys. 6:105-117.

The Diabetes Control and Complications Trial Research Group. 1993. The effect of intensive treatment of diabetes on the development and progression of long-term complications in insulin-dependent diabetes mellitus. The New England Journal of Medicine. 329:977-986.

B. Eyden and M. Tzaphlidou. 2001. Structural variations of collagen in normal and pathological tissues: role of electron microscopy. Micron. 32:287-300.

D. Eyre. 2002. Collagen of articular cartilage. Arthritis Research. 4:30-35.

S. Fine and W. P. Hansen. 1971. Optical second harmonic generation in biological tissues. Applied Optics. 10:2350-2353.

F. S. Foster, C. J. Pavlin, K. A. Harasiewicz, D. A. Christopher, and D. H. Turnbull. 2000. Advances in ultrasound biomicroscopy. Ultrasound in Medicine and Biology. 26:1-27.

P. A. Franken, A. E. Hill, C. W. Peters, G. Weinrich. 1961. Generation of optical harmonics. Physical Review Letters. 7:118-119.

I. Freund and M. Deutsch. 1986a. Second-harmonic microscopy of biological tissue. Optics Letters. 11:94-96. 
I. Freund, M. Deutsch, and A. Sprecher. 1986b. Connective tissue polarity: Optical second-harmonic microscopy, crossed-beam summation, and small-angle scattering in rat-tail tendon. Biophysical Journal. 50:693-712.

P. Friedl, E. B. Brocker, K. S. Zanker. 1998. Integrins, cell matrix interactions and cell migration strategies: fundamental differences in leukocytes and tumor cells. Cell Adhesion and Communication. 6:225-236.

E. Fukada. 1964. Japanese Journal of Applied Physics. 3:117-121.

J. N. Gannaway and C. J. R. Sheppard. 1978. Second-harmonic imaging in the scanning optical microscope. Optical and Quantum Electronics. 10:435-439.

R. Gauderon, P. B. Lukins, C. J. R. Sheppard. 1998. Three-dimensional second-harmonic generation imaging with femtosecond laser pulses. Optics Letters. 23:1209-1211.

R. Gauderon, P. B. Lukins, C. J. R. Sheppard. 2001a. Simultaneous multichannel nonlinear imaging: combined two-photon excited fluorescence and secondharmonic generation microscopy. Micron. 32:685-689.

R. Gauderon, P. B. Lukins, C. J. R. Sheppard. 2001b. Optimization of second-harmonic generation microscopy. Micron. 32:691-700.

E. Georgiou, T. Theodossiou, V. Hovhannisyan, K. Politopoulos, G. S. Rapti, and D. Yova. 2000. Second and third optical harmonic generation in type I collagen, by nanosecond laser irradiation, over a broad spectral region. Optics Communications. 176:253-260.

H. K. Graham, D. F. Holmes, R. B. Watson, and K. E. Kadler. 2000. Identification of collagen fibril fusion during vertebrate tendon morphogenesis. The process relies 
on unipolar fibrils and is regulated by collagen-proteoglycan interaction. Journal of Molecular Biology. 295:891-902.

Y. Guo, P. P. Ho, A. Tirksliunas, F. Lui, R. R. Alfano. 1996. Optical harmonic generation from animal tissues by the use of $\mathrm{ps}$ and fs laser pulses. Applied Optics. 35:68106813.

Y. Guo, P. P. Ho, H. Savage, D. Harris, P. Sacks, S. Schantz, F. Liu, N. Zhadin, and R. R. Alfano. 1997. Second-harmonic tomography of tissue. Optics Letters. 22:13231325.

Y. Guo, H. E. Savage, F. Liu, S. P. Schantz, P. P. Ho, and R. R. Alfano. 1999. Subsurface tumor progression investigated by noninvasive optical second harmonic tomography. Proceedings of the National Academy of Sciences, USA. 96:1085410856.

G. S. He and S. H. Liu. 1999. Physics of Nonlinear Optics. World Scientific Publishing Co., Singapore.

E. Heidemann. 1988. The chemistry of tanning. In Collagen: Biotechnology. Volume 3. Marcel E. Nimni, editor. CRC Press, Boca Raton. 39-61.

R. Hellwarth, and P. Christensen. 1974. Nonlinear optical microscopic examination of structure in polycrystalline ZnSe. Optics Communications. 12: 318-322.

V. Hovanessian and A. Lalayan. 1997. Second harmonic generation in biofibercontaining tissue. Proc. Int. Conf. Lasers. 96:107-109.

E. B. Hunziker. 1999. Articular cartilage repair: are the intrinsic biological constraints undermining this process insuperable? Osteoarthritis and Cartilage. 7:15-28. 
K. E. Kadler, D. F. Holmes, J. A. Trotter, and J. A. Chapman. 1996. Collagen fibril formation. Biochemical Journal 316:1-11.

W. Koechner. 1999. Mode locking. Solid-state laser engineering. Springer-Verlag, Berlin.

B-M Kim, J. Eichler, and L. B. Da Silva. 1999. Frequency doubling of ultrashort laser pulses in biological tissues. Applied Optics. 38:7145-7150.

B-M Kim, J. Eichler, K. M. Reiser, A. M. Rubenchik, L. B. Da Silva. 2000. Collagen structure and nonlinear susceptibility: effect of heat, glycation, and enzymatic cleavage on second harmonic signal intensity. Lasers in Surgery and Medicine. 27:329-335.

H. K. W. Kim, P. S. Babyn, K. A. Harasiewicz, K. P. H. Pritzker, and F. S. Foster. 1995. High resolution imaging of articular cartilage using ultrasound biomicroscopy. Journal of Orthopaedic Research. 13:963-970.

D. A. Kleinman, A. Ashkin, and G. D. Boyd. 1966. Second-harmonic generation of light by focused laser beams. Physical Review. 145:338-379.

K. König. 2000. Multiphoton microscopy in life sciences. Journal of Microscopy. 200:83-104.

T. H. Maiman. 1960. Nature. 187:493-.

D. J. Maitland and J. T. Walsh. 1997. Quantitative measurements of linear birefringence during heating of native collagen. Lasers in Surgery and Medicine. 20:310-318.

B. R. Masters, G. Gonnord, and P. Corcuff. 1997. Three-dimensional microscopic biopsy of in vivo human skin: a new technique based on a flexible confocal microscope. Journal of Microscopy. 185:329-338. 
L. Moreaux, O. Sandre, and J. Mertz. 2000. Membrane imaging by second-harmonic generation microscopy. Journal of the Optical Society of America B. 17:16851694.

L. Moreaux, O. Sandre, S. Charpak, M. Blanchard-Desce, and J. Mertz. 2001. Coherent scattering in multi-harmonic light microscopy. Biophysical Journal. 80:15681574.M. E. Nimni and R. D. Harkness. 1988. Molecular structures and functions of collagen. In Collagen: Biochemistry. Volume 1. Marcel E. Nimni, editor. CRC Press, Boca Raton. 1-77.

D. A. D. Parry and A. S. Craig. 1988. Collagen fibrils during development and maturation and their contribution to the mechanical attributes of connective tissue. In Collagen: Biochemistry and Biomechanics. Volume 2. Marcel E. Nimni, editor. CRC Press, Boca Raton. 1-23.

G. N. Ramachandran and G. Kartha. 1954. Structure of collagen. Nature. 174:269-270.

K. M. Reiser. 1991. Nonenzymatic glycation of collagen in aging and diabetes. Proceedings of the Society of Experimental Biology and Medicine. 196:17-29.

K. M. Reiser. 1998. Nonenzymatic glycation of collagen in aging and diabetes. Proceedings of the Society of Experimental Biology and Medicine. 218:23-37.

K. E. Rieckhoff and W. L. Peticolas. 1965. Optical second-harmonic generation in crystalline amino acids. Science. 147:610-611.

S. Roth and I. Freund. 1979. Second harmonic generation in collagen. Journal of Chemical Physics. 70:1637-1643.

S. Roth and I. Freund. 1980. Coherent optical harmonic generation in rat-tail tendon. Optics Communications. 33:292-296. 
S. Roth and I. Freund. 1981. Optical second-harmonic scattering in rat-tail tendon. Biopolymers. 20:1271-1290.

S. Roth and I. Freund. 1982. Second harmonic generation and orientational order in connective tissue: a mosaic model for fibril orientational ordering in rat-tail tendon. Journal of Applied Crystallography. 15:72-78.

A. Saied, E. Cherin, H. Gaucher, P. Laugier, P. Gillet, J. Floquet, P. Netter, and G. Berger. 1997. Assessment of articular cartilage and subchondral bone: subtle and progressive changes in experimental osteoarthritis using $50 \mathrm{MHz}$ echography in vitro. Journal of Bone and Mineral Research. 12:1378-1386.

N. T. Sanghvi, A. M. Snoddy, S. L. Myers, K. D. Brandt, C. R. Reilly, and T. D. Franklin. 1990. Characterization of normal and osteoarthritic cartilage using 25 $\mathrm{MHz}$ ultrasound. IEEE Ultrasonic Symposium Proceedings. 3:1413-1416.

K. Schoenenberger, B. W. Colston Jr., D. J. Maitland, L. B. Da Silva, and M. J. Everett. 1998. Mapping of birefringence and thermal damage in tissue by use of polarization-sensitive optical coherence tomography. Applied Optics. 37:60266036.

Y. R. Shen. 1984. The Principles of Nonlinear Optics. John Wiley \& Sons, New York.

J. Squier and M. Müller. 2001. High resolution nonlinear microscopy: a review of sources and methods for achieving optimal imaging. Review of Scientific Instruments. 72:2855-2867.

B. C. Stuart, M. D. Feit, S. Herman, A. M. Rubenchik, B. W. Shore, and M. D. Perry. 1996. Nanosecond-to-femtosecond laser-induced breakdown in dielectrics. Physical Review B. 53:1749-1761. 
S. Tanaka, G. Avigad, B. Brodsky, and E. F. Eikenberry. 1988. Glycation induces expansion of the molecular packing of collagen. Journal of Molecular Biology 203:495-505.

Z. S. Tannous, M. C. Mihm, T. J. Flotte, and S. Gonzalez. 2002. In vivo examination of lentigo maligna and malignant melanoma in situ, lentigo maligna type by nearinfrared reflectance confocal microscopy: comparison of in vivo confocal images with histologic sections. Journal of the American Academy of Dermatology. $46: 206-263$

T. Theodossiou, E. Georgiou, V. Hovhannisyan, and D. Yova. 2001. Visual observation of infrared laser speckle patterns at half their fundamental wavelength. Lasers in Surgery and Medicine 16:34-39.

T. Theodossiou, G. S. Rapti, V. Hovhannisyan, E. Georgiou, K. Politopoulos, and D. Yova. 2002. Thermally induced irreversible conformational changes probed by optical second harmonic generation and laser-induced fluorescence. Lasers in Medical Science 17:34-41.

J. Toyras, J. Rieppo, M. T. Nieminen. H. J. Helminen, and J.S. Jurvelin. 1999. Characterization of enzymatically induced degradation of articular cartilage using high frequency ultrasound. Physics in Medicine and Biology. 44:2723-2733.

L. S. Vasilenko, V. P. Chebotaev, and Y. V. Troitskii. 1965. Visual observation of infrared laser emission. Soviet Physics JETP. 21:513-514.

T. Verbiest, S. Van Elshocht, M. Kauranen, L. Hellemans, J. Snauwaert, C. Nuckolls, T. J. Katz, and A. Persoons. 1999. Strong enhancement of nonlinear optical properties through supramolecular chirality. Science. 282:913-915. 
J. D. Watson and F. H. C. Crick. 1953. Molecular structure of nucleic acids. Nature. 171:737-740.

T. J. Wess, A. P. Hammersley, L. Wess, and A. Miller. 1998. A consensus model for molecular packing of Type I collagen. Journal of Structural Biology 122:92-100.

R. M. Williams, W. R. Zipfel, and W. W. Webb. 2001. Multiphoton microscopy in biological research. Current Opinion in Chemical Biology. 5:603-608.

R. M. Wood. 1986. Laser Damage in Optical Materials. Hilger, Boston.

Y. Xia, J. B. Moody, N. Burton-Wurster, and G. Lust. 2001. Quantitative in situ correlation between microscopic MRI and polarized light microscopy studies of articular cartilage. Osteoarthritis and Cartilage. 9:393-406.

A. Zoumi, A. Yeh, and B. J. Tromberg. 2002. Imaging cells and extracellular matrix in vivo by using second-harmonic generation and two-photon excited fluorescence. Proceedings of the National Academy of Sciences. 99:11014-11019.

Chemistry of Collagen. 1967. G. N. Ramachandran, editor. Academic Press, New York. Collagen. 1988. Volumes 1-5. Marcel E. Nimni, editor. CRC Press, Boca Raton. 


\section{THEORY}

As discussed in Chapter 1, second harmonic generation is related to the second order nonlinear susceptibility tensor of a material. There are two important aspects of the second order nonlinear susceptibility tensor of collagen. The first is the ratio between the tensor elements; this determines the dependence of the second harmonic signal on the laser polarization. The second is the magnitude of the tensor elements-this plays an important role in determining the strength of the second harmonic signal generated. While the nonlinear susceptibility tensor is crucial in determining the amount of second harmonic light generated in a sample and its polarization dependence, other optical effects-birefringence, scattering, and interference between light generated at different points in the sample - can also play a role. In thin samples of relatively uniform tissue linear optical effects have little influence on the polarization dependence of the SHG signal. However, interference between second harmonic light generated at different points within the interaction volume has a strong effect on the strength of the second harmonic signal that is detected. In this section, we summarize the theory that allows us to understand the polarization dependence of the second harmonic signal as well as the variation in its strength.

\section{SHG in collagen: polarization dependence}

\section{Cylindrical symmetry model}

An analytical model for the polarization dependence of SHG in collagen fibers, based on the assumption of cylindrical symmetry, was developed previously (Roth and Freund, 1979; Roth and Freund, 1981; Freund et. al., 1986). The derivation of this 
model, in notation suited to the polarization-modulation technique used in this work, is presented below.

For any material with cylindrical symmetry $\left(C_{\infty}\right.$ symmetry), the most general vector expression for the second order nonlinear polarization is,

$$
\vec{P}=a \hat{s}\left(\hat{s} \cdot \vec{E}_{1}\right)^{2}+b \hat{s}\left(\vec{E}_{1} \cdot \vec{E}_{1}\right)+c \vec{E}_{1}\left(\hat{s} \cdot \vec{E}_{1}\right)
$$

where $\hat{s}$ represents the unit vector along the symmetry (fiber) axis, $\vec{E}_{1}$ is the incident electric field, and $a, b$, and $c$ are coefficients related to the second order nonlinear susceptibility tensor of the material. The nonlinear susceptibility tensor can then be written as

$$
\chi_{i j k}^{(2)}=a s_{i} s_{j} s_{k}+b s_{i} \delta_{j k}+c / 2\left(s_{j} \delta_{i k}+s_{k} \delta_{i j}\right) .
$$

In collagen, this tensor has additional symmetry (termed Kleinman symmetry) stemming from the fact that the second harmonic wavelength $(400 \mathrm{~nm}$ ) is far from the peak (at $\sim 270$ $\mathrm{nm}$ ) of the first electronic resonance ( $\mathrm{Li}$ et. al., 1997). When all of the frequencies involved in a nonlinear optical process are far below the first electronic resonance of a material, the nonlinear susceptibility can be taken to be real (no absorption) and independent of frequency. Under this condition, analysis of the quantum mechanical expressions for the nonlinear susceptibility tensor shows that the tensor has full permutation symmetry: all tensor elements that have indices that are permutations of each 
other are equal (e.g. $\chi_{i j k}=\chi_{j i k}=\chi_{k j i}=\chi_{k i j}=\chi_{i k j}=\chi_{j k i}$ ). Boyd, (1992), presents a more detailed discussion of Kleinman symmetry. In the case of cylindrical symmetry, the assumption of Kleinman symmetry simply leads to $b=c / 2$. Therefore, we can write,

$$
\vec{P}=a \hat{s}\left(\hat{s} \cdot \vec{E}_{1}\right)^{2}+b \hat{s}\left(\vec{E}_{1} \cdot \vec{E}_{1}\right)+2 b \vec{E}_{1}\left(\hat{s} \cdot \vec{E}_{1}\right)
$$

We further assume a Gaussian input beam (implicitly making the paraxial approximation) and assume the laser beam is normally incident on the sample. Under these assumptions, the input laser and generated second harmonic polarization is confined to the plane normal to the laser propagation direction $\hat{k}$. Given the nonlinear polarization, $\vec{P}$, we can write down expressions for the second harmonic field $E_{2||}$ generated along the fiber axis $(\hat{s})$ and the second harmonic field $E_{2 \perp}$ generated along the normal to that axis $(\hat{s} \times \hat{k})$ :

$$
\begin{gathered}
E_{2 \|}(\vec{r}, t)=A_{2||}(z) \hat{s} \frac{\exp \left(i\left(k_{2} z-\omega_{2} t\right)-r^{2} / w_{o}^{2}\left(1+i z / z_{R}\right)\right)}{1+i\left(z / z_{R}\right)} \\
E_{2 \perp}(\vec{r}, t)=A_{2 \perp}(z)(\hat{s} \times \hat{k}) \frac{\exp \left(i\left(k_{2} z-\omega_{2} t\right)-r^{2} / w_{o}^{2}\left(1+i z / z_{R}\right)\right)}{1+i\left(z / z_{R}\right)}
\end{gathered}
$$

where $A_{2 \mid}$ and $A_{2 \perp}$ are given by the following differential equations, respectively, 


$$
\begin{aligned}
& 2 i k_{2} \frac{\partial A_{2 \|}}{\partial z}=-\mu \omega_{2}^{2}(\vec{P} \cdot \hat{s}) \frac{e^{i \Delta k z}}{1+i z / z_{R}} \\
& 2 i k_{2} \frac{\partial A_{2 \perp}}{\partial z}=-\mu \omega_{2}^{2}(\vec{P} \cdot(\hat{s} \times \hat{k})) \frac{e^{i \Delta k z}}{1+i z / z_{R}}
\end{aligned}
$$

Here, $\omega_{2}$ and $k_{2}$ are the second harmonic frequency and wave number respectively, $r$ and $z$ are the radial and axial position respectively, $w_{o}$ and $z_{R}$ are the focused beam waist and Rayleigh range respectively, $\Delta k=2 k_{1}-k_{2}$ ( $k_{1}$ is the wave number at the laser frequency and $k_{2}$ the wave number at the second harmonic), and $\mu$ is the magnetic permeability. Refer to, for instance, Boyd (1992) for a detailed derivation of these expressions from Maxwell's equations. Neglecting the effect of linear birefringence ( $\Delta k$ is taken to be independent of the polarization direction) and polarization-dependent scattering, the polarization dependence of the second harmonic intensity (proportional to the square of the electric field) is contained entirely in the terms $\vec{P} \cdot \hat{s}$ and $\vec{P} \cdot(\hat{s} \times \hat{k})$. Thus, we can write,

$$
I_{S H G} \propto(\vec{P} \cdot \hat{s})^{2}+(\vec{P} \cdot(\hat{s} \times \hat{k}))^{2}
$$

Using Eq. 2.6, the dependence of the second harmonic signal on the polarization of the input laser light can be calculated. To simplify the discussion, a coordinate system where the laser beam is incident along the $z$-axis and polarized at an angle $\alpha$ with respect to the $\mathrm{x}$-axis is chosen. The fiber is located in the $\mathrm{x}-\mathrm{y}$ plane; we let it be oriented at an angle $\phi$ 
from the y-axis. This geometry is shown in Figure 2.1. Using this geometry, we can obtain an expression from Eq. 2.3 and Eq. 2.6 for the second harmonic intensity as a function of $\alpha$ and $\phi$.

$$
I_{S H G} \propto \frac{1}{8}\left(3+20 \gamma+40 \gamma^{2}\right)-\frac{1}{2}\left(1+6 \gamma+8 \gamma^{2}\right) \operatorname{Cos}(2 \alpha+2 \phi)+\frac{1}{8}(1+4 \gamma) \operatorname{Cos}(4 \alpha+4 \phi)
$$

Here we define $\gamma=b / a$; unlike a and $\mathrm{b}, \gamma$ can be determined without making any absolute intensity measurements. $\quad \gamma$ is a fundamental parameter of the second-order nonlinear susceptibility tensor-it is directly related to the ratio of the tensor's two independent elements. Figure 2.2 shows a theoretical curve of the second harmonic signal as a function of polarization angle, for a value of $\gamma=-0.65$ typical of the measurements made in collagen (refer to Chapter 7).

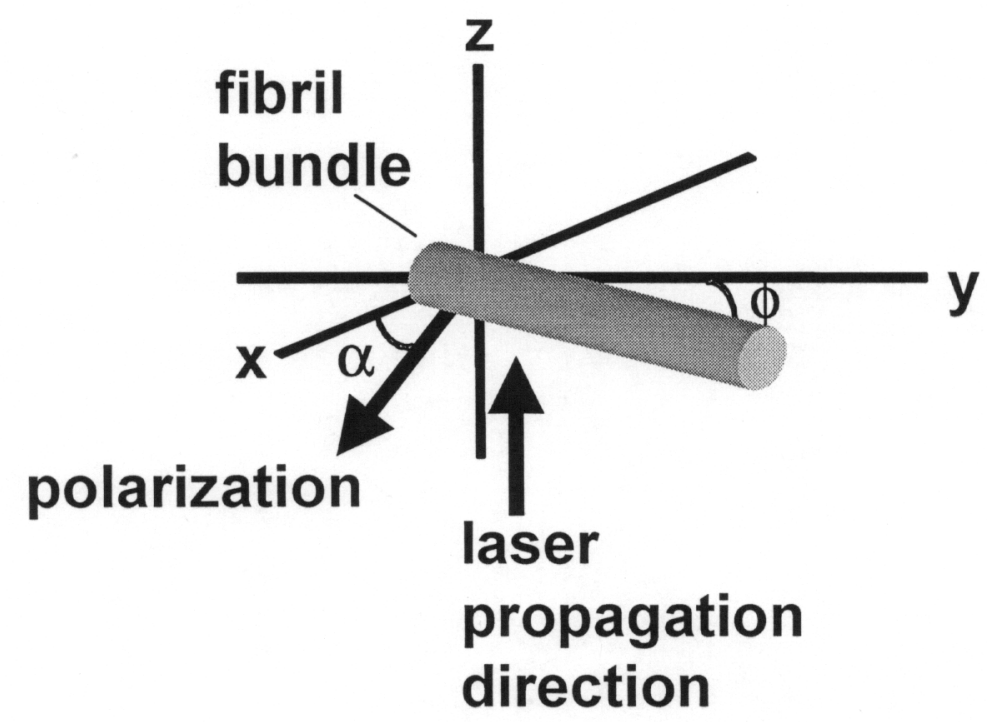

Figure 2.1. Sketch of fiber orientation and beam propagation direction and polarization. 


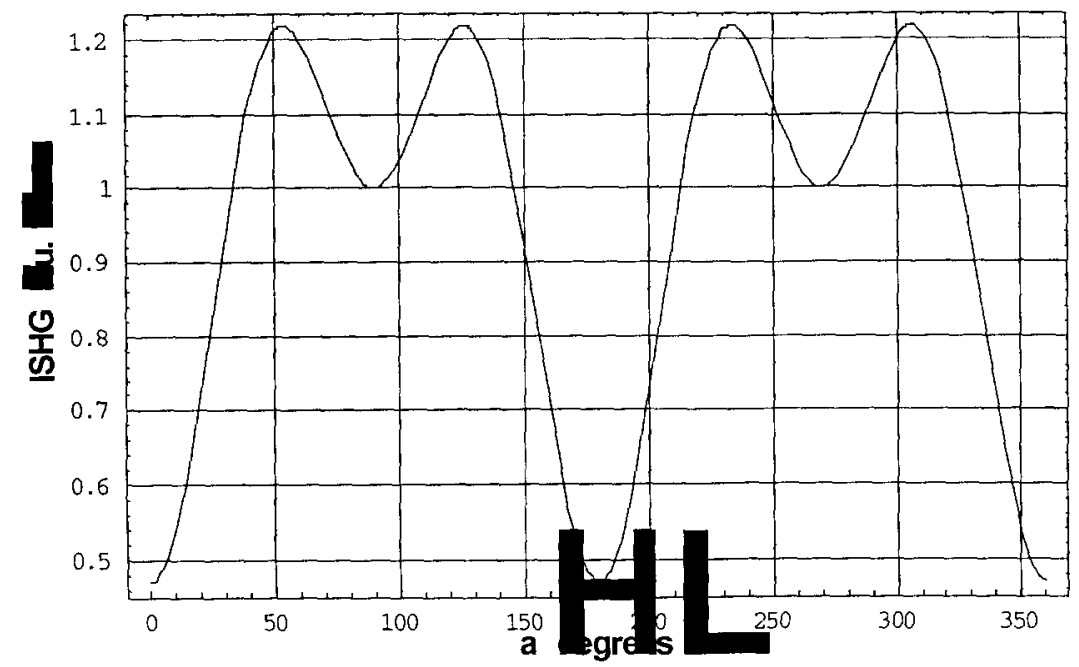

Figure 2.2. Theoretical plot of $I_{S H G}$ versus polarization angle $\alpha$, for a collagen fibril oriented at an angle $\phi=0$ degrees. A value of $\gamma=-0.65$, typical of the measurements made in collagen samples was used (refer to Chapter 7).

\section{Necessary conditions for validity of the model}

Equation 2.7 indicates a direct relationship between the observable polarization dependence of the second harmonic signal and $\gamma$. However, several assumptions are required for this relationship to hold. First, we have assumed that the beam is normally incident on the rat-tail tendon fascicle and that all of the fibers in the fascicle are perfectly aligned. Second, we have assumed that the effect of linear birefringence in the tissue on the polarization state of the input laser light can be neglected. For a given value of $\gamma$, what is the effect of relaxing each of these assumptions on the measured polarization dependence?

Calculating the effect of oblique incidence on the fiber is straightforward (refer to Figure 2.3). The generated second order polarization is simply split into a component along the propagation direction and a component normal to it; we assume that the component in the propagation direction does not contribute to the second harmonic light 
to a significant degree; this is strictly true only in the plane-wave approximation, but is a reasonably good approximation even for a focused beam (Huse et. al., 2001). Let $\psi$ be the angle between the laser propagation direction and the normal to the fiber. The result of this calculation is then an equation identical in form to Eq. 2.7, but $\gamma$ is replaced by $\gamma^{*}(\gamma, \psi):$

$$
I_{S H G} \propto \frac{1}{8}\left(3+20 \gamma^{*}+40 \gamma^{* 2}\right)-\frac{1}{2}\left(1+6 \gamma^{*}+8 \gamma^{* 2}\right) \operatorname{Cos}(2 \alpha+2 \phi)+\frac{1}{8}\left(1+4 \gamma^{*}\right) \operatorname{Cos}(4 \alpha+4 \phi)
$$

Since the form of Eq. 2.8 (oblique incidence) is identical to that of Eq. 2.7 (normal incidence), it is easy to measure the polarization dependence, calculate $\gamma^{*}$ from the measurements, and then mistakenly assume $\gamma=\gamma^{*}$. However, unless the laser beam is normally incident on a collagen fibril, $\gamma \neq \gamma^{*}$.

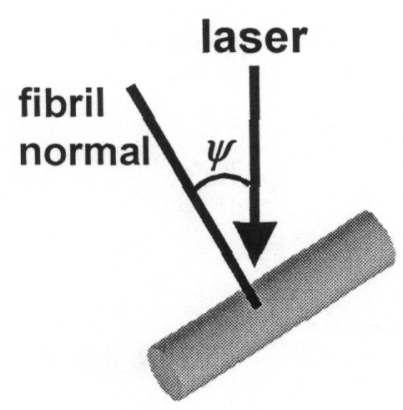

Figure 2.3. Oblique incidence on a collagen fibril. 
The calculated value of $\gamma^{*}$ as a function of $\psi$ is plotted in Figure 2.4 for a typical value of $\gamma=-0.65$. It should be noted that the effect of oblique incidence becomes less important as the absolute value of $\gamma$ increases.

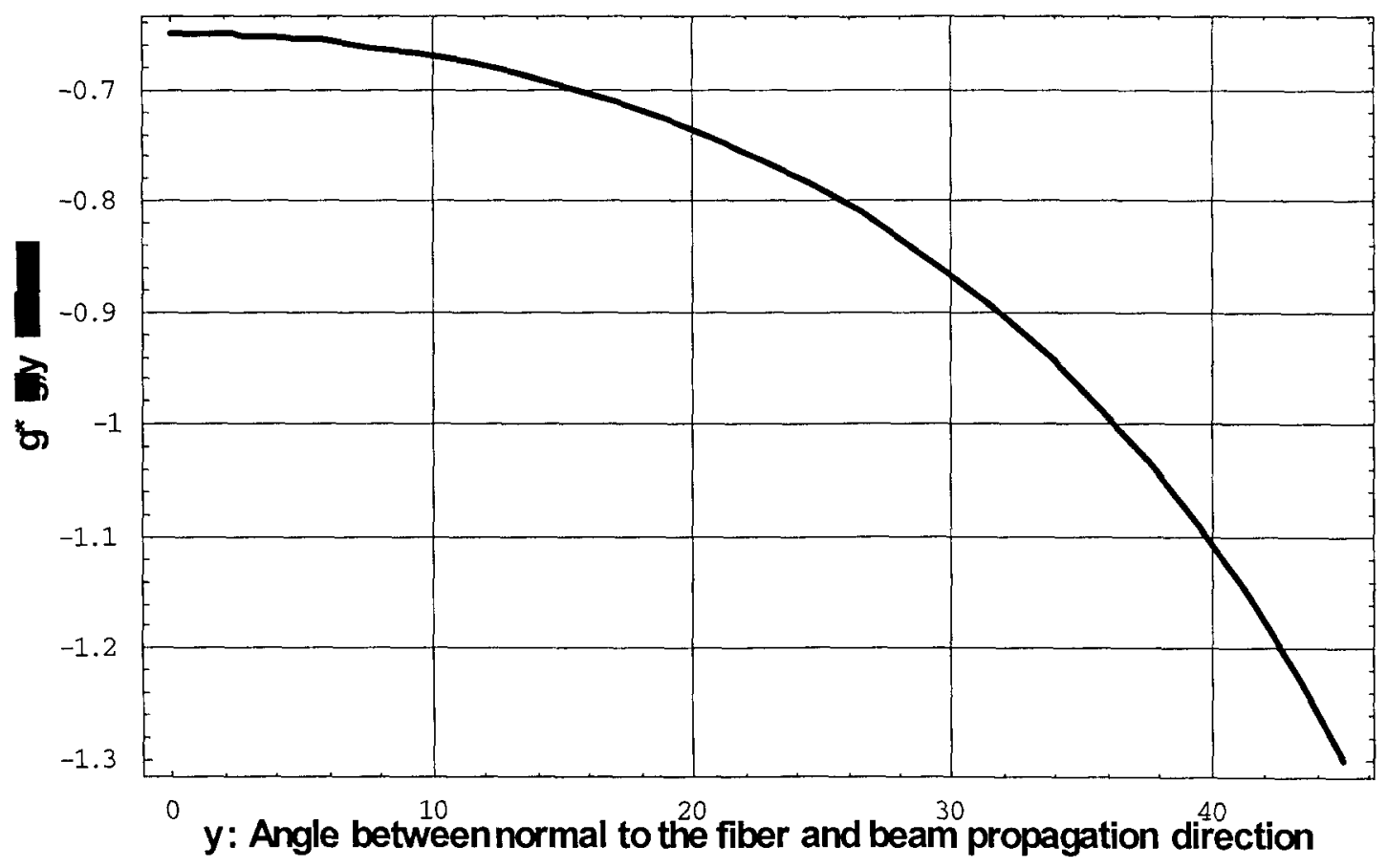

Figure 2.4. Plot of the effect of oblique incidence of laser beam on collagen fibrils on the value of $\gamma^{*}$ (for an actual value $\gamma=-0.65$ ).

A similarly simple calculation can be performed to take into account the presence of multiple fibers oriented at different angles within the focal spot of the laser beam. For illustration, Figure 2.5 shows the modified value of $\gamma$ for the simple case of two fibers oriented at varying angles $(\Delta \phi)$ with respect to each other (Figure 2.6). Again, the polarization dependence takes the same form as in Eq. 2.7 (normal incidence) except that $\gamma$ is replaced by $\gamma^{* *}(\gamma, \Delta \phi)$. 


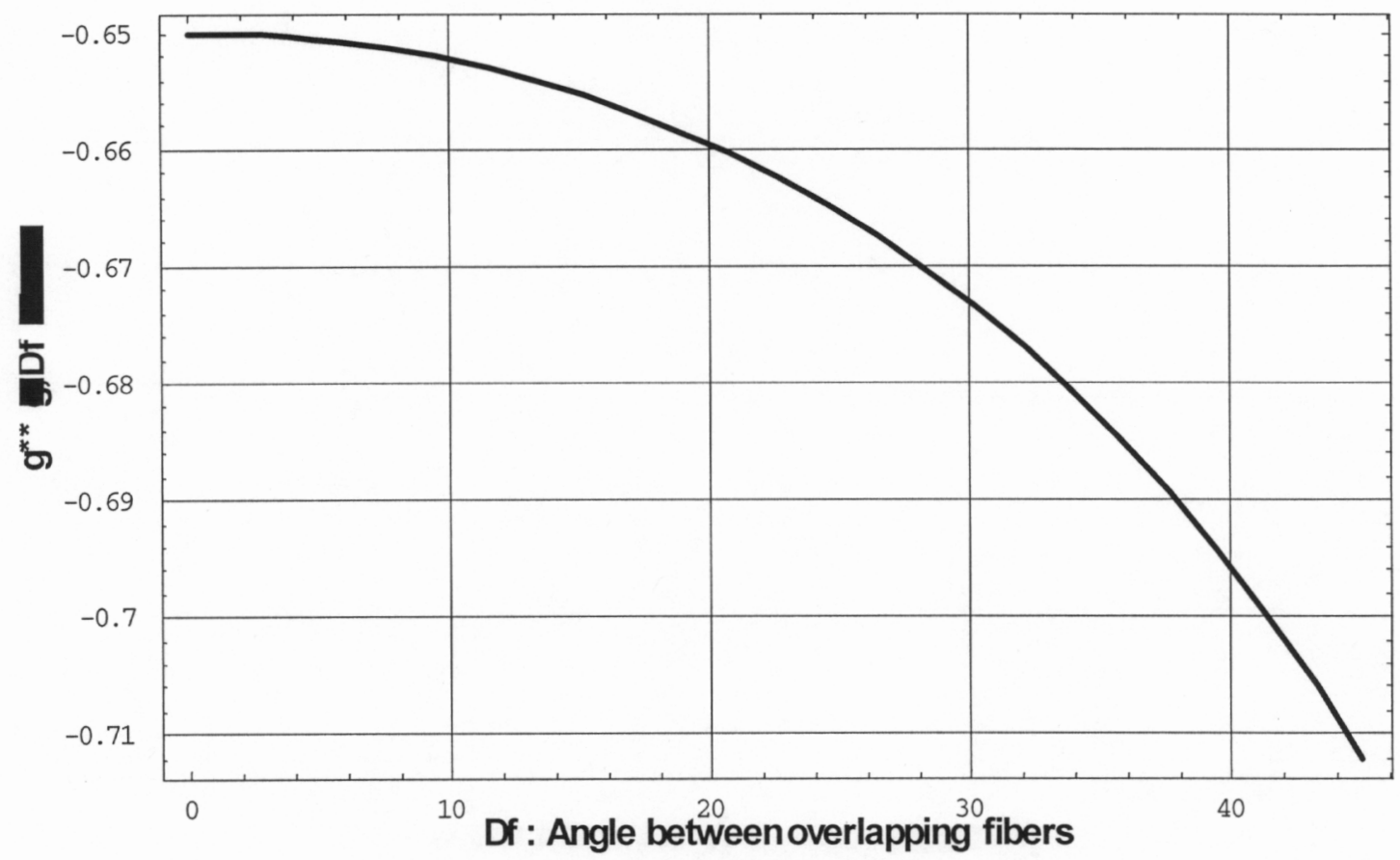

Figure 2.5. Plot of the effect of overlapping collagen fibrils of different orientation on the value of $\gamma^{* *}$ (for an actual value $\gamma=-0.65$ ).

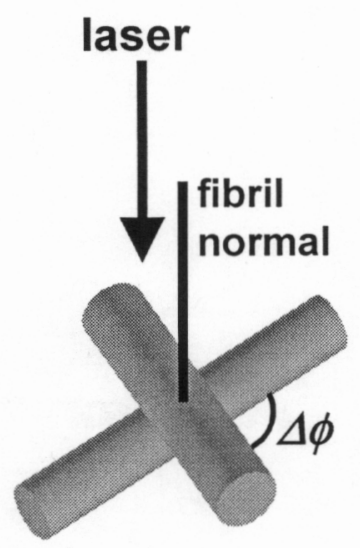

Figure 2.6. Overlapping collagen fibrils of different orientation.

Adding the effect of birefringence to our calculation is more complicated. The change in the measured ratio due to birefringence in a structure with uniform collagen orientation throughout depends on several parameters: the size of the focal volume, the 
depth of the focus in the material, and the degree of birefringence (which is sensitive to the index of refraction of the surrounding medium). Collagen is birefringent because the index of refraction for light polarized along the fiber axes is higher than that for light polarized perpendicular to the axis (intrinsic birefringence) and because of the cylindrical shape of the collagen fibrils embedded in a medium of lower refractive index (form birefringence). An arbitrary linearly polarized beam will transform into an elliptically polarized beam as it propagates into a birefringent medium. The change in polarization state must be incorporated into the equations that propagate the light through the nonlinear medium (Eqs. 2.4 and 2.5).

For simplicity, we use a new coordinate system with its axes defined relative to the collagen fibril. We choose the collagen fibril to be oriented along the $x$-axis. We must calculate both the component of the second harmonic signal $I_{\text {shgY }}$ polarized perpendicular to the fiber and the component $I_{\text {shgX }}$ polarized parallel to the fiber. The nonlinear susceptibility tensor allows three possibilities: (1) a component of the input beam along the fiber and a component normal to it can produce second harmonic light normal to the fiber, (2) a component of the input beam normal to the fiber can produce second harmonic light parallel to the fiber, and (3) a component of the input beam parallel to the fiber can produce second harmonic light parallel to the fiber. If we integrate Eq. 2.5 for each of these cases (taking into account the different wave vectors for different polarization directions), we have 


$$
\begin{aligned}
& I_{s h g Y} \propto\left|2 b A_{1 y} A_{1 x} \int_{-L}^{-L+S} \frac{\operatorname{Exp}\left[i\left(k_{1 y}+k_{1 x}-k_{2 y}\right)(z+L)\right.}{1+i \frac{z}{z_{R}}} d z\right|^{2} \\
& I_{s h g X} \propto\left|b A_{1 y}^{2} \int_{-L}^{L+S} \frac{\operatorname{Exp}\left[i\left(2 k_{1 y}-k_{2 x}\right)(z+L)\right.}{1+i \frac{z}{z_{R}}} d z+(a+3 b) A_{1 x}^{2} \int_{-L}^{-L+S} \frac{\operatorname{Exp}\left[i\left(2 k_{1 x}-k_{2 x}\right)(z+L)\right]}{1+i \frac{z}{z_{R}}} d z\right|^{2}
\end{aligned}
$$

In these expressions, $L$ represents the distance from the front surface to the focal point and $S$ refers to the sample thickness.

In Eqs. 2.9 and 2.10, $A_{l x}$ and $A_{l y}$ depend on the input polarization angle $\alpha$, and the Fourier components of $I_{s h g}=I_{s h g_{x}}+I_{s h g_{y}}$ are readily calculated to obtain the coefficients of three terms: a term $C_{0}$ independent of $\alpha$, a term $C_{I}$ proportional to $\operatorname{Cos}(2 \alpha+2 \phi)$ and a term $C_{2}$ proportional to $\operatorname{Cos}(4 \alpha+4 \phi)$. In order to determine the effect of birefringence, we compare the ratio $C_{2} / C_{1}$ and the ratio $C_{I} / C_{0}$ predicted by Eq. 2.7 (no birefringence) with the results of calculations that include birefringence. Figure 2.7 shows plots of the absolute value of these two ratios versus thickness in a sample with the beam focused at the center (we again take $\gamma=-0.65$ ), and Figure 2.8 illustrates the corresponding polarization dependence for a number of different values of the sample thickness. Note that polarization angle at which the peak signal occurs is not changed by propagation through a birefringent medium. Measurements of birefringence in collagen indicate that $\mathrm{n}_{\text {parallel }}-\mathrm{n}_{\text {perpendicular }} \sim 0.003$ (Bolin et. al., 1989; Poh, 1996; Maitland, 1995); for simplicity, we neglect dispersion and use this value in calculating the plots shown in 
Figure 2.7. The effect of birefringence will be smaller in tissues (such as cornea or intravertebral disk) where fibers are not aligned along one direction consistently throughout the depth of the tissue.

a.)

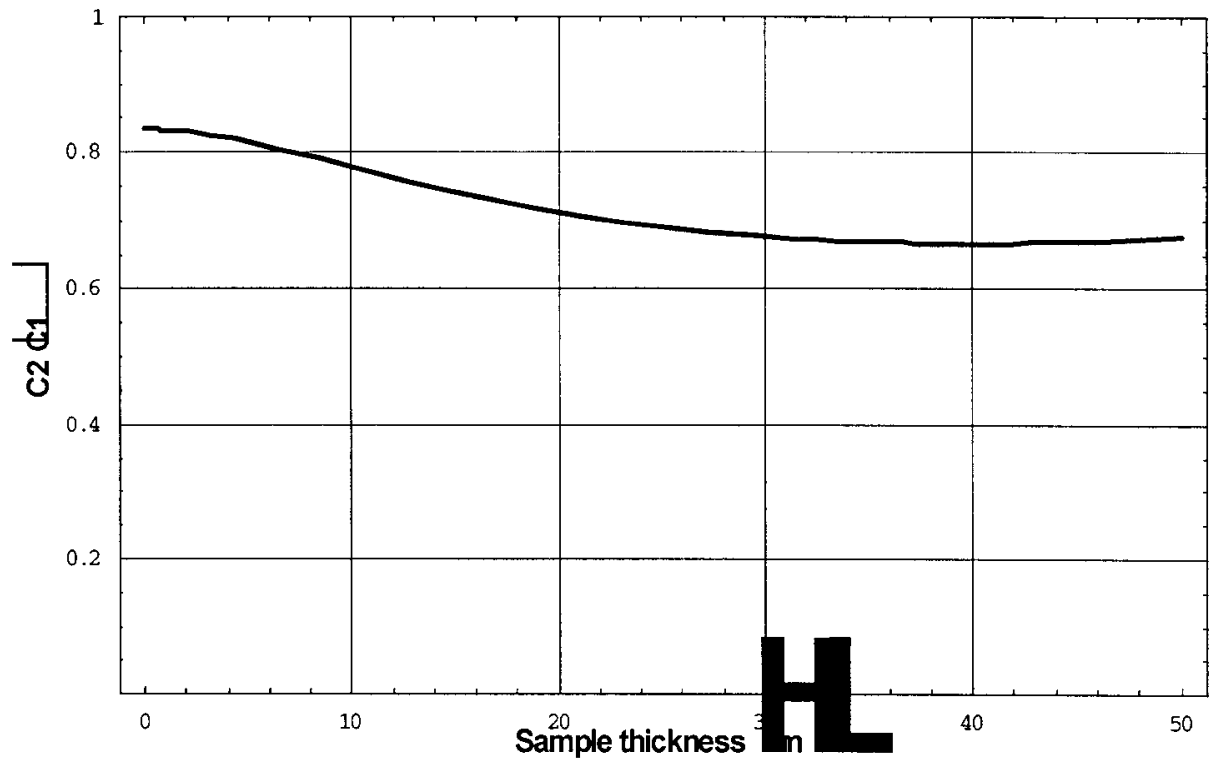

b.)

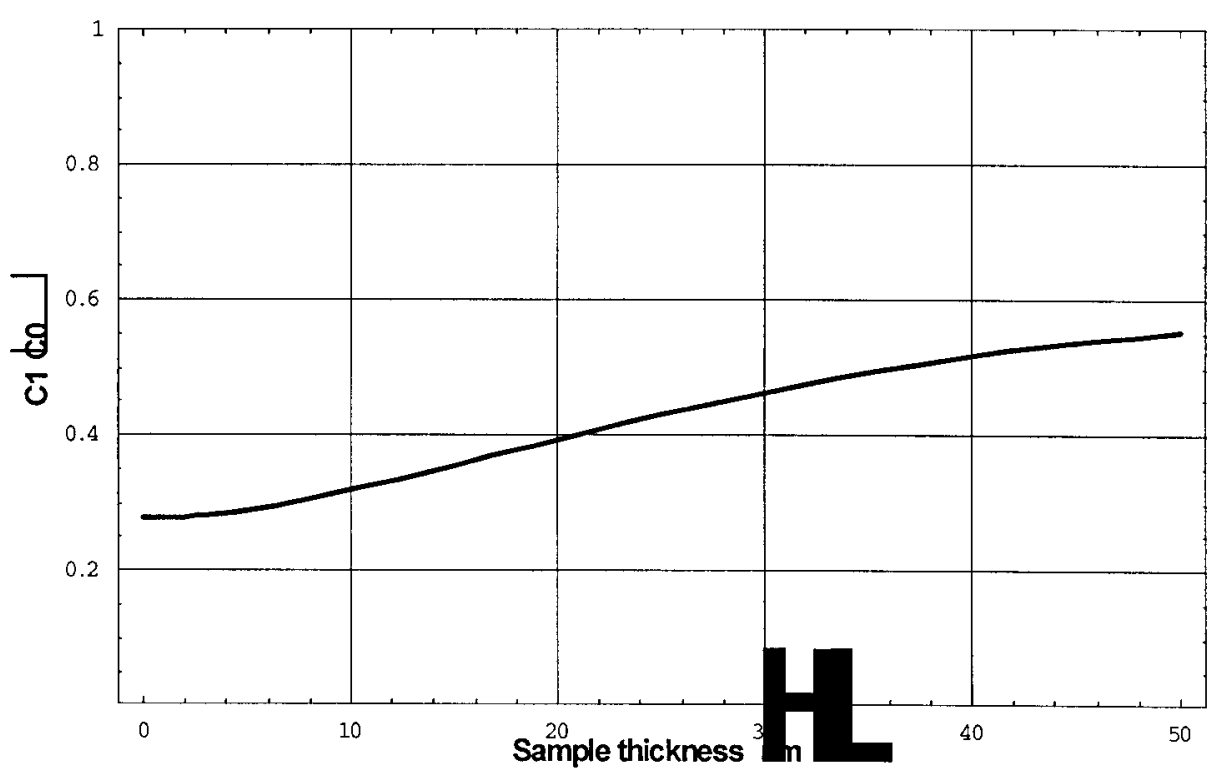

Figure 2.7. Plot illustrating the effect of birefringence on the polarization dependence of SHG in collagen. The ratio (a) $\mathrm{C}_{2} / \mathrm{C}_{1}$ and (b) $\mathrm{C}_{1} / \mathrm{C}_{0}$ is plotted versus the sample thickness. The ratio for a zero-thickness sample corresponds to the ratio that would be observed in a non-birefringent sample. 
a.)

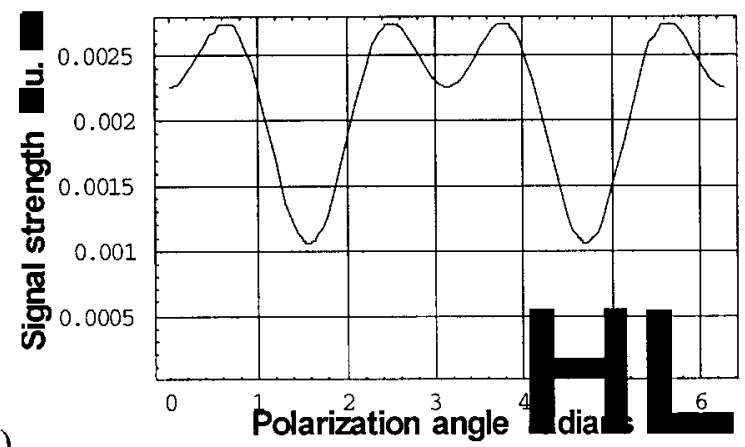

b.)

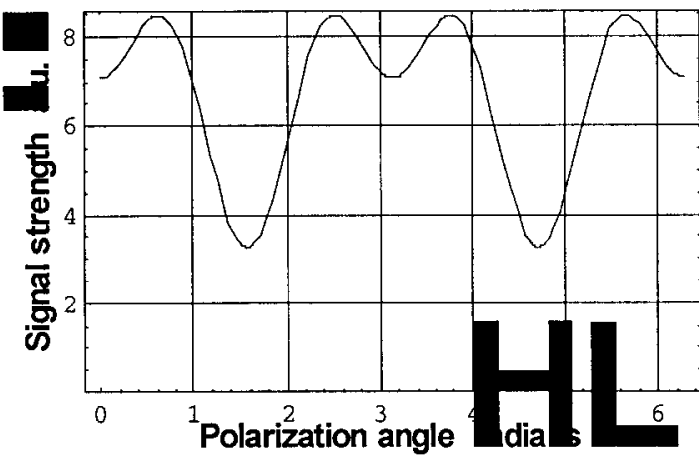

c.)

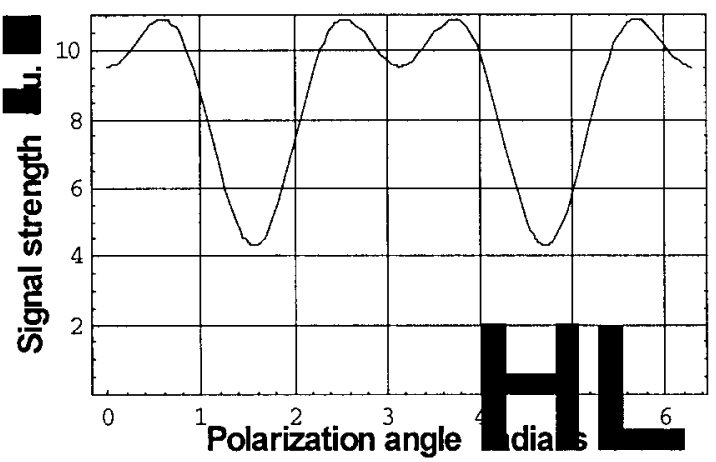


d.)

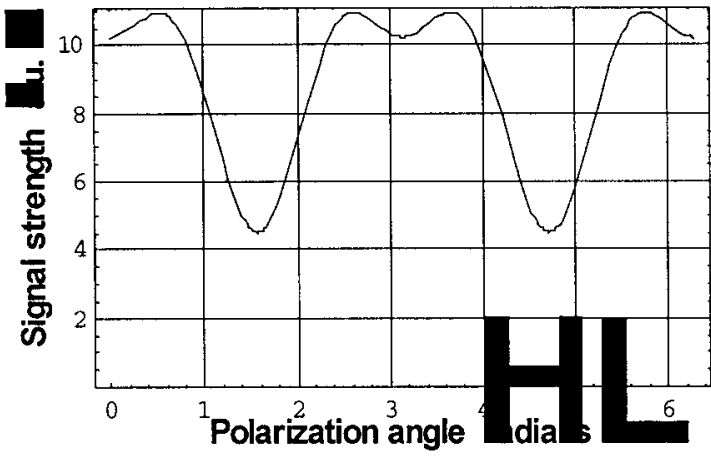

e.)

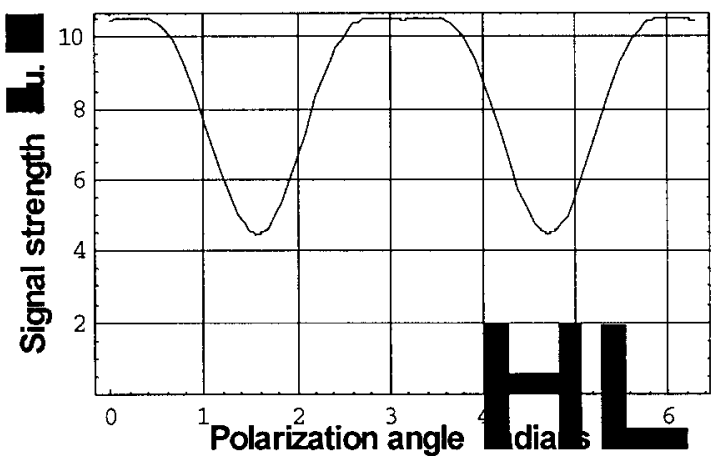

f.)

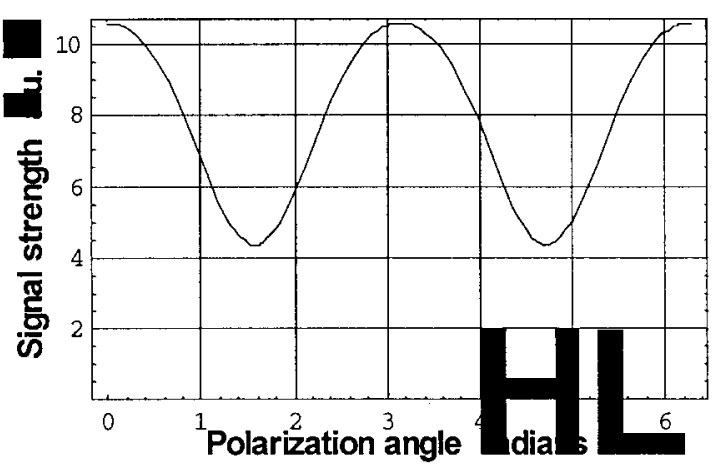

Figure 2.8. Plots illustrating the effect of birefringence on the polarization dependence of SHG in collagen, for different values of the sample thickness of (a.) $0 \mu \mathrm{m}$, (b.) $10 \mu \mathrm{m}$, (c.) $20 \mu \mathrm{m}$, (d.) $30 \mu \mathrm{m}$, (e.) $40 \mu \mathrm{m}$, and (f.) $50 \mu \mathrm{m}$. Note the fact that the polarization angle at which the peak signal occurs does not change. 


\section{SHG in collagen: signal strength}

Modeling the total power at the second harmonic frequency generated by a tightly focused laser beam propagating through a collagen sample is more difficult than modeling the polarization dependence. In this work, SHG is measured in thin $(\sim 5 \mu \mathrm{m})$ sections of highly organized rat-tail tendon, which consists of parallel bundles of collagen fibers, and this is the tissue we choose to model. The thickness of the sample, the properties of the focusing, and the dispersion of the collagen, among other factors, must be taken into account in calculating the total power generated. Unlike the polarization dependence, the total power generated also depends strongly on the relative polarity of neighboring fibrils. Two neighboring fibrils pointing in the same direction (with respect to the orientation of the $\mathrm{C}$ and $\mathrm{N}$ termini of their component molecules) will generate second harmonic fields that are in phase with one another and lead to enhanced SHG; on the other hand, two neighboring fibrils pointing in opposite directions will interfere destructively, leading to reduced SHG (Freund et. al., 1986). Several recent studies that imaged both SHG and two photon fluorescence have highlighted the importance of this effect in SHG microscopy of biological tissue. For instance, Campagnola et. al. (2002) observed cancellation of the SHG signal in tubulin structures, and this effect was also observed in styryl dye molecules attached to biological membranes (Campagnola et. al., 1999, Moreaux et. al., 2000, Moreaux et. al., 2001).

Dispersion in collagen leads to a difference in the index of refraction for light with a wavelength of $800 \mathrm{~nm}$ (laser wavelength, index of refraction $n_{l}$ ) and light with a wavelength of $400 \mathrm{~nm}$ (second harmonic wavelength, index of refraction $n_{2}$ ) of order $\Delta n$ $=-0.03$; the index of refraction of collagen is $\sim 1.5$ (Bolin et. al., 1989; Poh, 1996; 
Maitland, 1995). This gives a coherence length of order $l_{c}=7 \mu \mathrm{m}$, which is comparable to the $4 \mu \mathrm{m}$ thickness of the frozen sections we use. Therefore, if all of the fibrils in the interaction region are pointing the same way, the signal is strongly enhanced. If the fibril orientation is random, so that some fibrils point in one direction and others in the opposite direction, then the signal is significantly weaker.

Here we obtain a simple expression for the power of the second harmonic light generated assuming that all of the fibrils point in one direction - this gives a maximum value for the amount of second harmonic light that could be generated. We assume a Gaussian input laser beam focused to a diffraction-limited spot, with 1/e radius $w_{o}$ and Rayleigh range $z_{R}$. From Byer (1977), we have then,

$$
P_{2 \omega}=\frac{\pi w_{o}^{2}}{2} I_{2 \omega}
$$

where the second harmonic intensity is given by (from Boyd et. al., 1992),

$$
I_{2 \omega}=\frac{2 \omega^{2} d_{e f f}^{2} I_{\omega}^{2}\left|J\left(z_{R}, z_{f}, \Delta k\right)\right|^{2}}{n_{1}^{2} n_{2} c^{3} \varepsilon_{o}} .
$$

In this expression, $\omega$ is the frequency of the incident light, $d_{e f f}$ is the effective nonlinear susceptibility of collagen, $I_{\omega}$ is the input laser intensity, $n_{l}$ is the index of refraction of collagen at the laser frequency and $n_{2}$ is the index of refraction at the second harmonic frequency, $c$ is the speed of light in air, $\varepsilon_{o}$ is the permeability of free space, and 


$$
J\left(z_{R}, z_{f}, \Delta k\right)=\int_{-z_{f}}^{-z_{f}+L} \frac{\exp (i \Delta k z)}{1+i z / z_{R}} d z .
$$

The thickness of the sample is given by $L$ and the focal depth of the beam in the sample by $z_{f} ; \Delta k=2 k_{1}-k_{2}$ is the phase mismatch, where the wave vectors are defined as $k_{l}=$ $2 \pi n_{1} / \lambda_{1}$, and $k_{2}=2 \pi n_{2} / \lambda_{2}$.

As noted earlier, this calculation gives the maximum possible second harmonic power that can be detected in a collagen sample; it assumes that the entire sample volume is occupied by collagen, and that all of the fibrils are oriented in the same direction. Since rat-tail tendon is approximately $60 \%$ collagen by volume (Parry and Craig, 1977), the actual signal would be further reduced by about $40 \%$.

\section{SHG in collagen: back-scattering geometry}

SHG microscopy can be performed both by detecting transmitted second harmonic light and by detecting reflected second harmonic light. The experimental setup used in this work (refer to Chapter 4), allows both transmitted and reflected second harmonic light to be detected simultaneously. Reflection-mode SHG microscopy is necessary to image tissue in vivo. Thus, some of the issues specific to detecting SHG in the reflection geometry are summarized here. Reflected second harmonic light can be produced in two ways: it can be generated in the forward direction and back-scattered within the sample or at the boundary between the sample and glass slide; or, it can be generated in the backward direction. Second harmonic light generated in the backward direction is generally weak because of the absence of phase matching. While the 
coherence length for co-propagating second harmonic light is given by $l_{c}=\frac{2}{2 k_{1}-k_{2}}$, the coherence length for backward-propagating second harmonic light is much smaller: $l_{c}=\frac{2}{2 k_{1}+k_{2}} \cong \frac{1}{2 k_{1}}$.

For collagen, these values can be estimated as $l_{c} \sim 4 \mu \mathrm{m}$ (forward direction) and $l_{c}$ $\sim 40 \mathrm{~nm}$ (backward direction). At first glance, this would suggest that most of the backward propagating second harmonic light detected was generated in the forward direction and later back-scattered. However, in collagenous tissue, the length over which coherent SHG can occur is limited by the distance over which neighboring collagen fibrils have the same polarity, which, as noted in Chapter 1, is not well understood. This is further complicated by the large range $(15 \mu \mathrm{m}$ to $500 \mu \mathrm{m})$ and random distribution of collagen fibril diameter in rat-tail tendon. It is therefore possible that, in some regions of the rat-tail tendon, significant second harmonic light is generated in the back-scattering direction. Comparison of the second harmonic signal generated in the forward and backward direction may provide some information about parallel and anti-parallel alignment of fibrils, but this is very limited by the large variability and uncertainty in the back-scattering efficiency.

What effect can back-scattering have on the polarization dependence of the second harmonic signal? Second harmonic light generated directly in the backward direction should have the same dependence on the input laser polarization as light generated in the forward direction (refer back to Equation 2.6). However, second harmonic light that is generated in the forward direction and then back-scattered within the sample may have a different polarization dependence. This is because the scattering 
process, particularly in highly anisotropic structures such as collagen fibrils, can itself depend on the polarization. In thin samples, where the mean free path for scatteringtypical values in biological tissue range from about $1 \mathrm{~mm}$ to about $10 \mu \mathrm{m}$-greatly exceeds the sample thickness, this effect should be small. In fact, measurements of SHG polarization dependence obtained in thin samples in reflection mode did not differ significantly from those obtained in transmission mode (discussed in detail in Chapter 7). 


\section{References}

F. P. Bolin, L. E. Preuss, R. C. Taylor, and R. J. Ference. 1989. Refractive index of some mammalian tissues using a fiber optic cladding method. Applied Optics. 28:22972303.

R. G. Byer. 1977. Parametric Oscillators and Nonlinear Materials. In Nonlinear Optics. P. G. Harper and B. S. Wherrett, editors. Academic Press, London. 47-160.

P. J. Campagnola, M. Wei, A. Lewis, and L. M. Loew. 1999. High-resolution nonlinear optical imaging of live cells by second harmonic generation. Biophysical Journal. $77: 3341-3349$.

P. J. Campagnola, A. C. Millard, M. Terasaki, P. E. Hoppe, C. J. Malone, and W. A. Mohler. 2002. Three-dimensional high-resolution second-harmonic generation imaging of endogenous structural proteins in biological tissues. Biophysical Journal. $81: 493-508$.

I. Freund, M. Deutsch, and A. Sprecher. 1986. Connective tissue polarity: Optical second-harmonic microscopy, crossed-beam summation, and small-angle scattering in rat-tail tendon. Biophysical Journal. 50:693-712.

N. Huse, A. Schönle, S. W. Hell. 1991. Z-polarized confocal microscopy. Journal of Biomedical Optics. 6:273-276.

Y-H. Li, J-C. Yue, and G-P. Cai. 1997. Fluorescence characterization of type I collagen from normal and silicotic rats and its quenching dynamics induced by hypocrelin B. Biopolymers. 42:219-226.

D. J. Maitland. 1995. "Dynamic measurements of tissue birefringence: Theory and experiments," Ph.D. Dissertation, Northwestern University, Evanston, IL. 
L. Moreaux, O. Sandre, and J. Mertz. 2000. Membrane imaging by second-harmonic generation microscopy. Journal of the Optical Society of America B. 17:16851694.

L. Moreaux, O. Sandre, S. Charpak, M. Blanchard-Desce, and J. Mertz. 2001. Coherent scattering in multi-harmonic light microscopy. Biophysical Journal. 80:15681574.

D. A. D. Parry, and A. S. Craig. 1977. Quantitative electron microscope observations of the collagen fibrils in rat-tail tendon. Biopolymers. 16:1015-1031.

D. T. Poh. 1996. Examination of refractive index of human epidermis in-vitro and invivo. Proc. Inter. Conf. Lasers '96. 118-125.

S. Roth and I. Freund. 1979. Second harmonic generation in collagen. Journal of Chemical Physics. 70:1637-1643.

S. Roth and I. Freund. 1981. Optical second-harmonic scattering in rat-tail tendon. Biopolymers. 20:1271-1290. 


\section{POLARIZATION-MODULATED SECOND HARMONIC GENERATION}

\section{Collagen}

In Chapter 2, an expression for the polarization dependence of the second harmonic light generated in thin, well-aligned samples of collagen fibrils oriented normal to the laser beam was derived (Eq. 2.7):

$$
I_{S H G} \propto \frac{1}{8}\left(3+20 \gamma+40 \gamma^{2}\right)-\frac{1}{2}\left(1+6 \gamma+8 \gamma^{2}\right) \operatorname{Cos}(2 \alpha+2 \phi)+\frac{1}{8}(1+4 \gamma) \operatorname{Cos}(4 \alpha+4 \phi)
$$

In this expression, $\gamma$ refers to a parameter of the second order nonlinear susceptibility tensor, $\alpha$ is the direction of the laser polarization, and $\phi$ is the orientation of the collagen fibrils.

\section{Measuring $\gamma$}

The most direct way to measure the parameters in Eq. 3.1 is to rotate the polarization $\alpha$ of the input laser light to different orientations (using a half-wave plate, for example) and to measure the second harmonic intensity at each orientation. To determine $\gamma$ and $\phi$, a minimum of three separate measurements is necessary, and additional measurements could be used to improve the accuracy of the technique. If images of $\gamma$ and $\phi$ are to be obtained, this technique would require at least three separate scans at three different polarization orientations; all three images would need to be in perfect 
registration. In this work, a more efficient technique to image these parameterspolarization modulation-is described.

The laser beam polarization is continuously rotated by driving an electro-optic modulator followed by a quarter-wave plate with a saw-tooth pattern at frequency $\Omega$, so that $\alpha=\Omega \pi$ (see Chapter 4 for details of the experimental technique). Thus, we can rewrite Eq. 3.1 as

$$
I_{S H G} \propto \frac{1}{8}\left(3+20 \gamma+40 \gamma^{2}\right)-\frac{1}{2}\left(1+6 \gamma+8 \gamma^{2}\right) \operatorname{Cos}(2 \pi \Omega t+2 \phi)+\frac{1}{8}(1+4 \gamma) \operatorname{Cos}(4 \pi \Omega t+4 \phi)
$$

It is apparent from this equation that signal is present at frequencies $2 \pi \Omega t$ and $4 \pi \Omega t$; we will call these the first modulation harmonic and second modulation harmonic (FMH and SMH), respectively. A lock-in amplifier is capable of measuring both the amplitude and phase of a signal at a reference frequency as well as at harmonics of the reference frequency. The signal at the FMH is proportional to

$$
I_{F M H} \propto-\frac{1}{2}\left(1+6 \gamma+8 \gamma^{2}\right)
$$

while the signal at the SMH is proportional to

$$
I_{S M H} \propto \frac{1}{8}(1+4 \gamma)
$$


The ratio $R$ of the amplitude of the signal at the first and second modulation harmonic can thus be written as

$$
R=-1 / 4+8 \gamma
$$

Equation 3.5 shows that $\gamma$ can be rapidly measured using amplitude measurements from two lock-in amplifiers. Furthermore, the intensity of the second harmonic signal at the FMH and SMH is independent of the fiber orientation.

It will be useful in our calculations to relate $\gamma$ to the elements of the second order nonlinear susceptibility tensor in a coordinate system with its axes fixed with respect to the collagen fibril. If we let the collagen fibrils be aligned parallel to the X-axis $(\phi=90$ degrees) and choose the Z-axis as the laser beam propagation direction in this coordinate system we find that

$$
d_{X Y Y}=\gamma d X X / 3 \gamma+1
$$

where $d_{X X X}$ and $d_{X Y Y}$ represent the two independent elements of the second order nonlinear susceptibility tensor $\left(d_{X Y Y}=d_{Y X Y}=d_{Y Y X}\right)$. It is possible to show from Eq. 3.1 that $d_{X X X}^{2} \propto 1+6 \gamma+9 \gamma^{2}$, and so

$$
d_{X X X}^{2}=\frac{2\left(1+6 \gamma+9 \gamma^{2}\right)}{1+6 \gamma+8 \gamma^{2}} d_{F M H}^{2}
$$


Here we have defined $d_{F M H}$ as the effective second order nonlinear susceptibility for the component of the signal at the first modulation harmonic.

By chopping the incident laser beam using an optical chopper, we can also measure the polarization-independent component of the signal. We will use $S$ to denote the ratio of the signal at the first modulation harmonic of the electro-optic modulator to the signal at the optical chopper frequency $S$. We should note that $S$ does not correspond exactly to the ratio of the first two terms in Eq. 3.2 above. The optical chopper produces a modulation that includes a DC component as well as components at the first and higher harmonics of the chopper frequency. If we rewrite Eq. 3.2 to include the effect of chopping the beam and we let $\Omega_{\text {chop }}$ be the chopper frequency, $C_{D C}$ the DC component of the chopped beam, and $C_{\Omega_{c h o p}}$ the component at the chopper frequency, we have (leaving out terms at frequencies other than $\Omega_{\text {chop }}$ and $\Omega$ )

$$
I_{S H G} \propto \frac{C_{\Omega_{c k p p}}}{8}\left(3+20 \gamma+40 \gamma^{2}\right) \operatorname{Cos}\left(2 \pi \Omega_{c h o p} t\right)-\frac{C_{D C}}{2}\left(1+6 \gamma+8 \gamma^{2}\right) \operatorname{Cos}(2 \pi \Omega t+2 \phi)+\frac{C_{D C}}{8}(1+4 \gamma) \operatorname{Cos}(4 \pi \Omega t+4 \phi)
$$

Thus, $S$ must be multiplied by a correction factor $C_{\Omega_{c h o p}} / C_{D C}$ to obtain the correct value for the ratio of the first two terms in Eq. 3.2. The exact value of this correction factor depends on the profile of the beam and the chopper blades. Rather than calculating the correction factor, we use results in quartz, which has a known second order nonlinear susceptibility, to measure it directly (refer to Chapter 5). We can also derive a relation analogous to Eq. 3.7: 


$$
d_{X X X}^{2}=\frac{8\left(1+6 \gamma+9 \gamma^{2}\right)}{3+20 \gamma+40 \gamma^{2}} d_{F M H}^{2},
$$

where $d_{c h o p}$ is defined as the effective second order nonlinear susceptibility for the component of the signal at the chopper frequency.

\section{Measuring orientation}

All of the information about fibril orientation is conveyed by the phase of the FMH and SMH signal (refer back to Eq. 3.2). A lock-in amplifier measuring the phase at the FMH will measure $2 \phi$ and a lock-in amplifier measuring the phase at the SMH will measure $4 \phi$. Thus, half of the phase of the FMH signal is equal to the fiber orientation, $\phi$. The phase of the SMH signal provides redundant information about the fiber orientation, but the phase difference between twice the FMH phase and the SMH phase (which must be 0 or 180 degrees) gives the sign of the ratio $R$. Refer to Figure 3.1 for a diagram illustrating the use of polarization modulation and phase-sensitive detection to determine fiber orientation. 

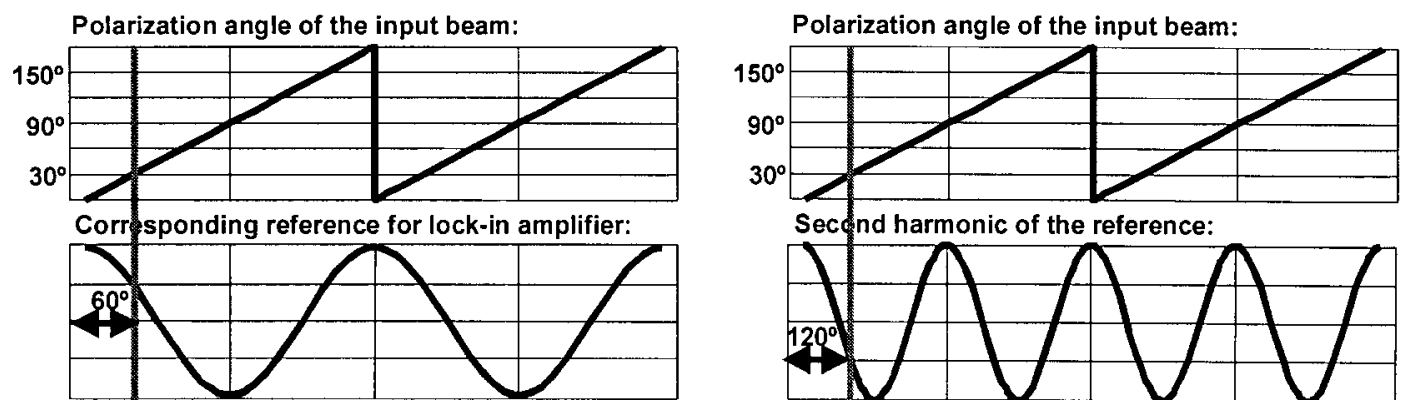

Second harmonic of the reference:

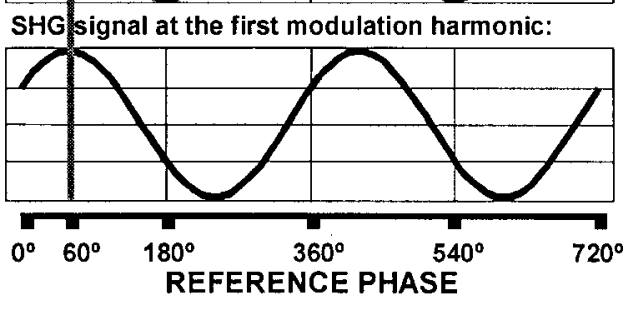

\section{TIME}

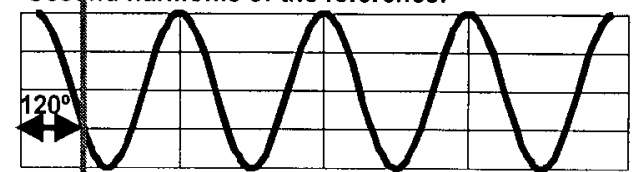

SHG signal at the second modulation harmonic:

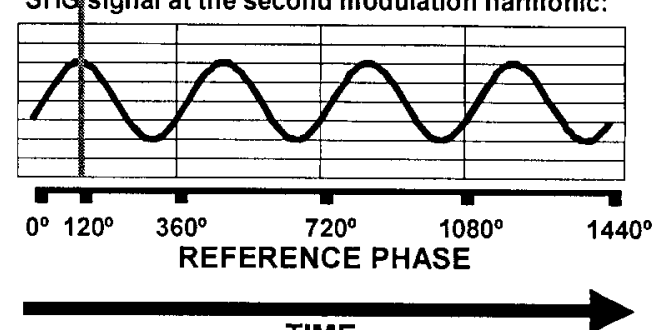

TIME

Figure 3.1. Sketch of the technique used to obtain fiber orientation information using polarization-modulation of the input laser beam. For convenience, the collagen fiber is assumed to be oriented at an angle of 30 degrees from the y-axis; it is also assumed that $R$ $=0.5$. All plots have a common time axis. The case for the FMH of the signal is shown on the left and the case for the SMH of the signal is shown on the right. The red line is drawn through the curves at the point in time when the input beam is polarized in the direction of the fiber; the SHG signal peaks at this time. From the plots, it is evident that twice the orientation angle is equal to the phase shift of the signal at the FMH and four times the orientation angle is equal to the phase shift of the signal at the SMH. 


\section{Quartz}

To independently confirm the accuracy of the polarization-modulation technique for measuring $R$ and $S$ in collagen, we can apply it to a well-characterized nonlinear crystal. We choose a quartz waveplate - a crystal cut where the optical axis lies in the plane of the waveplate, normal to the beam propagation direction. The second-order nonlinear susceptibility tensor in quartz (choosing the Z-axis as the optic axis) is given by

$$
d_{X X X(\text { quarz })}=-d_{X Y Y(\text { quarz })}=-d_{Z X Y(\text { quart })}=-d_{Z Y X(\text { quartz })}
$$

where $d_{X X X(q u a r z)}=0.40 \mathrm{pm} / \mathrm{V}($ Boyd, 1992) and all of the other tensor elements are zero. The surface of the waveplate lies in the X-Z plane; the laser propagates in the Ydirection. Using Eq. 3.10 and the fact that the second order nonlinear polarization is given by $P=\ddot{\chi} \cdot \vec{E} \cdot \vec{E}$, it is possible to obtain an expression for the second harmonic signal generated by the quartz plate as a function of polarization angle $\alpha$ (measured from the $\mathrm{X}$-axis):

$$
I_{S H G(q u a r z)} \propto \frac{3}{8}+\frac{1}{2} \operatorname{Cos}(2 \pi \alpha)+\frac{1}{8} \operatorname{Cos}(4 \pi \alpha)
$$

From Eq. 3.11, it is clear that the ratio $R$ between the signal at the second modulation harmonic and the signal at the first modulation harmonic should be 0.25 and the ratio $S_{\text {corr }}$ between the signal at the first modulation harmonic and the DC component of the signal (after correction for the effect of the chopper modulation itself, see above) should be 4/3. 
The measurements of the polarization dependence of SHG in quartz using this technique are presented and analyzed in Chapter 5.

It will be useful to relate the results of the polarization modulation measurements to the elements of the nonlinear susceptibility tensor in the coordinate system defined above. Substituting $\alpha=0$ (x-polarized input light) into Eq. $3.11\left(I_{S H G(\text { quariz })} \propto 1\right)$ gives the relative second harmonic signal corresponding to $d_{X X X(\text { quarz })}^{2}$. Thus,

$$
d_{X X X(\text { quartz })}^{2}=2 d_{F M H(\text { quartz })}^{2},
$$

where $d_{F M H}$ is the effective second order nonlinear susceptibility for the first modulation harmonic of the signal generated in quartz. Similarly, we have

$$
d_{X X X(\text { quartz })}^{2}=\frac{8}{3} d_{c h o p(q u a r z)}^{2}
$$




\section{References}

R.W. Boyd. 1992. Nonlinear Optics. Academic Press, San Diego. 


\section{EXPERIMENT}

\section{Optical setup for polarization-modulated SHG microscope}

The experimental setup used for polarization-modulated SHG microscopy in biological tissue samples is illustrated in Figure 4.1. A Ti:Sapphire oscillator (Mira; Coherent Inc., Santa Clara, CA) was used to generate linearly polarized $\sim 200$ fs pulses at a wavelength of $800 \mathrm{~nm}$, with a maximum energy of $5 \mathrm{~nJ}$, and at a repetition rate of 76 MHz. A Faraday isolator directly behind the laser shutter prevented back-scattered light from entering the laser cavity and interfering with mode-locking. Because the linear polarization of the laser is modulated after the isolator, it is not possible simply to use a passive optical isolator (which produces circularly polarized light).

The beam then passed through a half-wave plate followed by a polarizing beamsplitter; the half-wave plate was rotated to control the power incident on the sample. The p-polarized light transmitted through the beam-splitter was chopped at $1.5 \mathrm{kHz}$ using an optical chopper and then passed through an electro-optic modulator (360-80; Conoptics, Danbury, CT) with its axes oriented at 45 degrees to the polarization of the light. The beam was passed through a spatial filter and beam expander: a $50 \mathrm{~mm}$ plano-convex lens focused the beam onto a $50 \mu \mathrm{m}$ pinhole; a second lens placed behind the pinhole was used to collimate the light. Plano-convex singlet lenses with focal lengths of $50 \mathrm{~mm}, 100$ $\mathrm{mm}$, and $200 \mathrm{~mm}$ were used to expand the beam to different diameters. After the beam expander, the beam passed through a quarter-wave plate with its axes oriented at 45 degrees to those of the electro-optic modulator. 


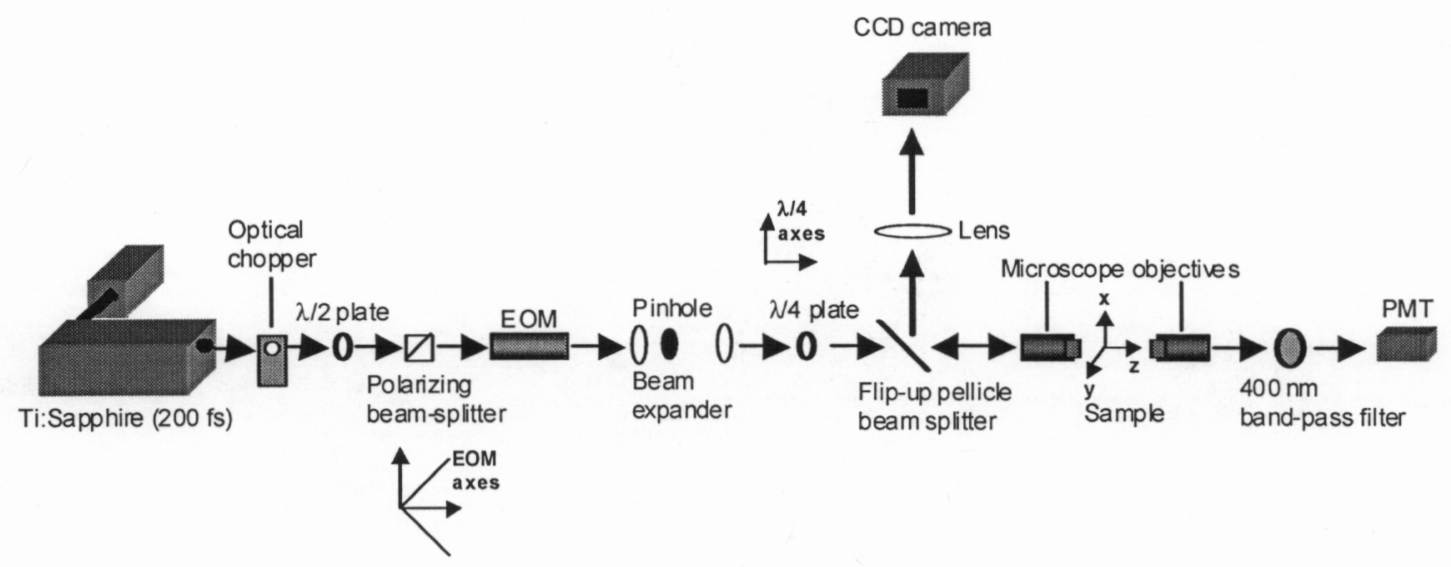

Figure 4.1. Experimental setup for polarization-modulated SHG microscopy

Several different microscope objectives (see Table 4.1) were used to focus the laser beam onto the sample; they will be referred to henceforth as the $10 \mathrm{X}, 20 \mathrm{X}$, and $40 \mathrm{X}$ objectives. The use of different microscope objectives and different beam expander collimating lenses allowed the numerical aperture of the system to be adjusted. An iris was used to allow only the central maximum of the Airy pattern produced by the spatial filter to enter the back-aperture of the microscope objectives. None of the microscope objectives significantly clipped the beam.

Table 4.1. Microscope objectives used in the experiment

\begin{tabular}{llll}
\hline $\begin{array}{l}\text { Nominal } \\
\text { magnification }\end{array}$ & Manufacturer/type & $\begin{array}{l}\text { Numerical } \\
\text { aperture }\end{array}$ & $\begin{array}{l}\text { Working } \\
\text { distance }\end{array}$ \\
\hline $10 \mathrm{X}$ & $\begin{array}{l}\text { Mitutoyo plano- } \\
\text { apochromat }\end{array}$ & 0.28 & $33.5 \mathrm{~mm}$ \\
\hline $20 \mathrm{X}$ & $\begin{array}{l}\text { Mitutoyo plano- } \\
\text { apochromat }\end{array}$ & 0.42 & $20.0 \mathrm{~mm}$ \\
\hline $40 \mathrm{X}$ & Nikon s fluor & 0.90 & $0.3 \mathrm{~mm}$ \\
\hline
\end{tabular}


The transmitted second harmonic signal was collected using a Mitutoyo $100 \mathrm{X}$ $(\mathrm{NA}=0.7$, working distance $=6.0 \mathrm{~mm})$ objective - other objectives did not have a suitably long working distance to use in this setup. This resulted in some undercollection of the SHG signal generated by the $40 \mathrm{X}$ objective $(\mathrm{NA}=0.9)$. A laser power meter was inserted in the beam path after the microscope objective in order to measure the average power of the laser beam; we determined that the fractional transmission of all three objectives was the same to within the uncertainty of the power measurement.

An interference filter with a peak transmission of $\sim 50 \%$ (at $\sim 400 \mathrm{~nm}$ ), a full width at half maximum of $\sim 40 \mathrm{~nm}$, and about four orders of magnitude of rejection at the fundamental frequency of the laser was used to reject the first harmonic and allow only the second harmonic signal to reach the photomultiplier tube (H6780; Hamamatsu Photonics K.K., Hamamatsu City, Japan) or channel photomultiplier (MH1944, PerkinElmer Optoelectronics, Santa Clara, CA) used for signal detection. In both cases, a cathode was chosen that was sensitive to light with wavelengths between $\sim 300 \mathrm{~nm}$ and $\sim 650 \mathrm{~nm}$; this provided an additional four or five orders of magnitude of rejection at the fundamental laser frequency. In order to minimize stray light, the detector was mounted in a light-tight box with holes for the second harmonic light and the electric cables. Optimum results were obtained with all room lights switched off, but, because of lock-in amplification, measurements could be made with dim lighting switched on.

A fiber-lamp (used while not scanning to illuminate the sample through the collecting objective), pellicle-beam splitter (flipped into the beam path before the focusing microscope objective when not scanning), lens, and CCD camera were used in order to image the sample and the focal spot of the laser. Imaging of the focal spot 
allowed optimization of the alignment and it also permitted appropriate regions of the sample for second harmonic imaging to be found.

\section{Polarization modulation}

The electro-optic modulator (EOM) and quarter-wave plate combination were used to rotate the polarization direction of the linearly polarized laser light. An EOM functions as a variable wave plate where the phase delay is proportional to the voltage applied across it. For linearly polarized input light, the EOM produces elliptically polarized light. The quarter-wave plate that followed the EOM converted the elliptically polarized light into linearly polarized light rotated by some angle with respect to the laser polarization direction. The degree of rotation was directly proportional to the applied voltage: from 0 degrees for $0 \mathrm{~V}$ to 180 degrees for the full-wave voltage of the EOM. The voltage across an EOM can be modulated at high frequencies; our system was limited to a bandwidth of $1 \mathrm{MHz}$.

A function generator (DS345; Stanford Research Systems, Sunnyvale, CA) provided a saw-tooth waveform at $4 \mathrm{kHz}$ that was amplified to have an amplitude equal to the full-wave voltage of the modulator using a high voltage power supply (Conoptics 302A). The polarization-modulated beam produced a modulated SHG signal in the sample with frequency components at the first and second harmonic of the modulation frequency (the origin of these modulations is discussed in Theory, below) After passing through a current preamplifier (SR570; Stanford Research Systems, Sunnyvale, CA), the modulated signal from the detector was decoded using three digital signal processing (DSP) lock-in amplifiers (SR830; Stanford Research Systems., Sunnyvale, CA) set to 
detect the amplitude and phase of the SHG signal at both the first and second harmonic of the EOM modulation frequency as well as at the optical chopper frequency.

\section{Beam steering mirrors}

The experimental setup described above does not use any beam steering mirrors. Dielectric mirrors, which can be designed to have the same reflectivity for s- and ppolarized light at a given wavelength over a bandwidth at least comparable to that of an ultra-short pulse laser $(\sim 30 \mathrm{~nm})$, generally introduce a phase delay between the s- and $\mathrm{p}$ components. Therefore, unless incident linearly polarized light is s- or p-polarized, it is converted into elliptically polarized light (see sketch in Figure 4.2). Metallic mirrors have a different reflectivity coefficient for s- and p-polarized light; except at near-normal incidence, they cannot be used without introducing substantial errors.

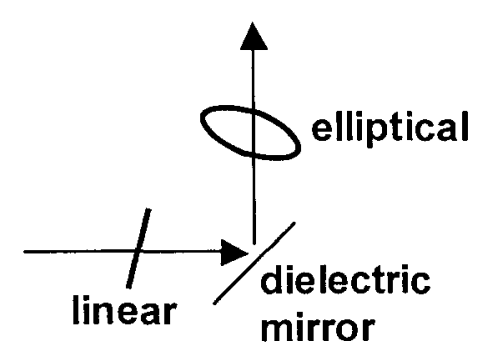

Figure 4.2. Sketch of effect of dielectric mirror on linearly polarized light

Dielectric turning mirrors can be used. One possibility for using them, of course, is to eliminate mirrors between the polarization-modulating optics and the sample. However, the small aperture of the EOM and the increase in the laser beam diameter with distance propagated precludes this in the experimental setup discussed above. Another, 
more practical solution, is to place the quarter-wave plate after all of the mirrors. This solution only works if the EOM and quarter-wave plate are rotated by 45 degrees and the polarization of the light entering the EOM is also rotated by 45 degrees (using a halfwave plate, for instance). The EOM is now oriented so that it introduces a phase delay between the s- and p-components of the polarization. In this case, the phase shift introduced by the mirrors simply adds to the phase shift introduced by the EOM and can be compensated by adjusting the DC offset voltage of the EOM to cancel it.

\section{Reflection-mode microscopy}

The polarization dependence of the second harmonic signal can also be measured in reflection (or "back-scattering") mode. For these measurements, the system was modified slightly to allow both transmission mode and reflection mode imaging. A dichroic mirror that transmitted the laser fundamental wavelength $(800 \mathrm{~nm})$ and reflected the second harmonic light $(400 \mathrm{~nm})$ was inserted after the quarter-wave plate. The mirror was chosen to have transmission and reflection independent of polarization. A second interference filter and photomultiplier tube (MH982, PerkinElmer Optoelectronics, Santa Clara, CA) were inserted to filter and collect the reflected second harmonic light. Two additional DSP lock-in amplifiers (EG\&G PARC 7260, Workingham, United Kingdom) were used to decode the reflection-mode signal.

\section{Two photon fluorescence}

For some experiments, it was useful to compare the second harmonic signal with the two photon fluorescence signal. At an excitation wavelength of $800 \mathrm{~nm}$, collagen 
does not exhibit two photon fluorescence (Zoumi et. al., 2002); however, two photon fluorescence does occur when the Ti:Sapphire laser is tuned to a wavelength of $740 \mathrm{~nm}$. To measure two-photon fluorescence, a $450 \mathrm{~nm}$ to $600 \mathrm{~nm}$ colored glass band-pass filter replaced the narrow-band $400 \mathrm{~nm}$ dielectric filter. Because the two photon fluorescence signal was significantly weaker than the SHG signal, it was detected in transmission mode while the SHG signal was detected in reflection mode (using a $370 \mathrm{~nm}$ narrowband dielectric filter).

\section{Group velocity dispersion}

The Ti:Sapphire ultra-short pulse oscillator used produces near transform-limited pulses of $\sim 200$ fs duration. However, group velocity dispersion in the optics traversed can result in broadening of femtosecond pulses. Squier and Müller (2002) reported that for $\sim 100$ fs pulses propagation through typical microscope objectives used in nonlinear microscopy results in an approximate doubling of the pulse length. The second harmonic (or two photon fluorescence) signal is thus reduced by approximately a factor of two. Other optical elements-such as lenses, polarizers, and waveplates-are also dispersive and can result in pulse broadening; however, they are much thinner and in most cases less dispersive than microscope objectives. Propagation through the tissue sample itself can lead to pulse broadening, although the fact that the maximum depth at which the samples are imaged is $\sim 300 \mu \mathrm{m}$ means that this is likely a very small contribution.

In the experimental setup described above, the electro-optic modulator can also introduce pulse broadening. Using the Sellmeier equations for lithium tantalate (Abedin and Ito, 1996), the pulse width after propagation through the $80 \mathrm{~mm}$ modulator was 
calculated for an input pulse width of $200 \mathrm{fs}$. This resulted in slightly less than a doubling of the pulse width. It is important to note that, because the pulse width is given by $\Delta T^{2}=\Delta T_{o}^{2}+\frac{(4 \log 2)^{d^{2} k} / d \omega^{2}{ }^{2}}{\Delta T_{o}^{2}} \quad$ (z represents the distance propagated through the material), the longer the initial pulse length $\Delta T_{o}$, the smaller the relative broadening (refer, for instance, to Siegman, 1986). Thus, group velocity dispersion compensation, while very important for $\Delta T_{o} \sim 10 \mathrm{fs}$, is not necessary in the case where $\Delta T_{o} \sim 200 \mathrm{fs}$. Since relatively long pulses were used and ample second harmonic signal strength was obtained in the collagen structures studied, pre-compensation for the group velocity dispersion in the system was not necessary.

\section{Image acquisition}

A Labview program was used to coordinate motion of the translation stage using a motion controller (ESP300; Newport, Irvine, CA) and Newport 850F actuators and to acquire data from a 16 channel PCI-MIO-16E-1 data acquisition board (National Instruments, Austin, TX) connected to the output of the lock-in amplifiers. A total of 10 data channels is necessary to simultaneously digitize the amplitude and phase output of the three lock-in amplifiers used to analyze the transmission-mode signal and the two lock-in amplifiers used to analyze the reflection-mode signal. The sample was moved in steps along one axis (with a maximum resolution of $1 \mu \mathrm{m}$ ) and scanned along a second axis to obtain two-dimensional images. To avoid problems with hysteresis in the translation stage and in the actuators driving it, data was collected only as the sample was scanned in one direction; the stage was returned to its starting position on the scan axis 
before the next scan. The start of the scan was coordinated with the start of data collection. The actual scan distance slightly exceeded the nominal image dimension to allow the actuators to accelerate to a constant speed before beginning data collection. The scan speed (limited by the actuators to $400 \mu \mathrm{m} / \mathrm{s}$ ) was chosen so that a single pixel in the image would be traversed in a time equal to three times the lock-in amplifier time constant. Typical time constants used ranged from $10 \mathrm{~ms}$ to $300 \mathrm{~ms}$, depending on the signal strength obtained in a given sample.

\section{Data analysis}

The data obtained using Labview were processed using software developed in Matlab (The Mathworks, Champaign-Urbana, IL). This software plotted the magnitude of the SHG signal as a function of two-dimensional position using false-color or shades of gray. The program could be instructed to ignore data points below a certain threshold-typically chosen to correspond to the noise level.

Orientation information was obtained from the phase data (from the first modulation harmonic channel) by adding an appropriate phase shift and dividing the result in half (refer to the previous chapter). The resulting information was plotted as a vector plot, using lines of uniform length. No orientation data were plotted at points where the magnitude of the signal at the first modulation harmonic was below the noise threshold.

The $R$ and $S$ ratios (and other ratios of the signal intensity at different modulation frequencies) could be plotted as a function of position. The Matlab program developed could also be used to produce histograms of the $R, S$, intensity, and orientation data. 
Average values and standard deviations of the $R$ and $S$ measurements were also calculated. 


\section{References}

K. S. Abedin and H. Ito. 1996. Temperature-dependent dispersion relation of ferroelectric lithium tantalite. Journal of Applied Physics. 80:6561-6563.

A. E. Siegman. 1986. Linear pulse propagation. Lasers. University Science Books, Sausalito, California. 331-361.

J. Squier and M. Müller. 2001. High resolution nonlinear microscopy: a review of sources and methods for achieving optimal imaging. Review of Scientific Instruments. 72:2855-2867.

Y. Yeh. 1990. Optical ellipsometry: theory and biophysical application. In Optical Methods in Biophysics. Alfred F. Leung, editor. The Chinese University Press, Hong Kong. 143-245.

A. Zoumi, A. Yeh, and B. J. Tromberg. 2002. Imaging cells and extracellular matrix in vivo by using second-harmonic generation and two-photon excited fluorescence. Proceedings of the National Academy of Sciences. 99 :11014-11019. 


\section{TESTING AND CALIBRATION OF THE EXPERIMENTAL SETUP}

The experimental setup described in Chapter 4 was tested and calibrated using known samples before it was applied to studying the second order nonlinear optical properties of tissue. Several potential sources of error were examined. First, the maximum average laser power level at which tissue could be examined without sustaining structural damage was determined for each microscope objective. Unintentional use of laser power levels exceeding the damage threshold could result in artificially low SHG signals. Second, the polarization-modulation technique was tested; the polarization state of the light was characterized to determine (1) that the light was in fact linearly polarized and (2) that the intensity of the modulated laser light did not vary with polarization angle. If these conditions were not met, artifacts could be introduced into the data. Finally, a quartz waveplate with known second order nonlinear susceptibility was used to determine if other, unanticipated sources of error existed in the experimental setup. The quartz waveplate was also used to calibrate the system to allow measurement of the magnitude of the second order nonlinear susceptibility. 


\section{Testing}

\section{Determining the damage threshold}

Although the use of ultra-short pulse lasers has vastly expanded the types of samples that can be investigated using nonlinear optics, damage to the sample is still an important problem. Above a certain threshold, the tightly focused laser beam used for SHG microscopy can damage the sample. To determine the maximum intensity that could be used, the damage threshold was measured in thin $(5 \mu \mathrm{m})$ frozen sections of rattail tendon-the sample used for most of the studies in this work-for the three different microscope objectives used in the experiment. The damage threshold was not measured in the other samples studied, but no damage was observed in these samples at the laser intensities used to image rat-tail tendon sections. Damage was defined as the presence of a change in structure sufficient to cause a loss of second order nonlinear susceptibility; subsequent inspection using white light microscopy always confirmed that the tissue had been significantly altered.

For each microscope objective, the samples were exposed to an initial laser intensity well below that at which damage was known to occur. After pausing five seconds at each power level, the half-waveplate that controlled the laser power reaching the sample was adjusted by a 2 degree increment to increase the power. The second harmonic signal at each power level was noted; a drop in the signal indicated damage had occurred. This process was repeated at 15 different points on the rat-tail tendon section for each microscope objective. The average laser power was measured before the microscope objective using a laser power meter, and corrected for losses in the microscope objective ( $\sim 70 \%$ transmission). The peak intensity of the laser pulses was 
calculated assuming Gaussian pulses $\left(I \propto \operatorname{Exp}\left[-2 t^{2} / \tau^{2}\right]\right.$ ) with a width of $\tau=200$ fs. The focal spot size for each microscope objective was calculated from measurements in quartz described in detail below; at the power levels used in this experiment, selffocusing is unlikely to affect the focal radius (Schaffer et. al., 2001).

For the 10X objective, no damage was observed for $65 \mathrm{~mW}$ average laser power (corresponding to peak intensity levels up to $\sim 50 \mathrm{GW} / \mathrm{cm}^{2}$ ), the maximum value attainable at the focus with the laser and experimental setup used. With the $20 \mathrm{X}$ objective, damage was first observed at a range of laser powers from $30 \mathrm{~mW}$ to $60 \mathrm{~mW}$ (corresponding to peak intensities between $\mathrm{I} \sim 100 \mathrm{GW} / \mathrm{cm}^{2}$ and $\mathrm{I} \sim 200 \mathrm{GW} / \mathrm{cm}^{2}$ ). Using the $40 \mathrm{X}$ objective, damage was first observed over a range of peak intensities: between $\mathrm{I} \sim 130 \mathrm{GW} / \mathrm{cm}^{2}$ and $\mathrm{I} \sim 160 \mathrm{GW} / \mathrm{cm}^{2}$ (about 20 to $25 \mathrm{~mW}$ average laser power).

In all of the scans described in subsequent chapters, the laser intensity was kept at least $25 \%$ below the damage thresholds given here. In almost all cases, damage occurred very rapidly, as soon as the power level was increased to the damage threshold level. Increasing the length of time a point on the sample was exposed to intensities below the damage threshold rarely led to damage. In addition, damage was usually accompanied by emission of a visible spark of white light from the sample.

The precise mechanism of damage was not investigated in these studies. However, the presence of a visible spark of white light accompanying the loss of SHG suggests the mechanism may be a nonlinear process (photoablation: a multi-photon ionization leading to electron avalanche and plasma formation) rather than a thermal mechanism. Although most studies of photoablation using femtosecond pulses have used microjoule pulses at $1 \mathrm{kHz}$ (or less) repetition rates (Stuart et. al., 1996; Noack and 
Vogel, 1999; Oraevsky et. al., 1996), ablation by tightly focused nanojoule pulses at 1 $\mathrm{kHz}$ in transparent dielectrics (Schaffer et. al., 2001) or by tightly focused nanojoule pulses at $80 \mathrm{MHz}$ in biological tissue (König et. al., 2002) has also been demonstrated. 


\section{Testing the polarization-modulation technique}

\section{Characterizing the incident light polarization}

In Chapter 3, a polarization-modulation technique for measuring $R$ and $S$, two parameters related to the second order nonlinear susceptibility tensor of a sample, was discussed. To modulate the polarization, an electro-optic modulator (EOM) and quarterwave plate were used. In theory, after passing through these optics, the light should be linearly polarized at an angle between 0 and 180 degrees that varies with time as the sawtooth wave applied across the EOM. However, in practice, slight misalignment of or imperfections in the polarizing optics can prevent the light from being linearly polarized or result in an intensity that depends on the polarization angle. To characterize the polarization of the incident light, a Glan-Taylor polarizer was placed behind the collecting microscope objective, and light transmitted by the polarizer was collected using a photodiode.

In the first test of the system, the voltage across the EOM was varied in small steps to adjust the polarization angle from 0 to 360 degrees in 5 degree increments. The photodiode signal was decoded using lock-in amplification at the chopper frequency and recorded; an example is plotted in Figure 5.1a. According to theory, the transmitted light intensity as a function of polarization angle $\alpha$ for a perfect polarizer should be given by

$$
I \propto 1+\operatorname{Cos}(2 \alpha)
$$

The theoretical curve is also shown in Figure 5.1a; there is very good agreement between measurement and theory. The Glan-Taylor polarizer was rotated to a number of different 
angles and the experiment was repeated with very similar results (Figure 5.1b presents one example). The ratio of the maximum value to the minimum value recorded is the extinction coefficient; the larger the extinction coefficient, the more purely linear the polarization state of the light. The lower the extinction coefficient, the more elliptically polarized the light is (refer to Figure 5.2). Since the electric field is proportional to the square root of the intensity, an extinction coefficient of 100 means that $10 \%$ of the electric field is polarized orthogonal to the nominal polarization direction.

Extinction coefficients between 300 and 850 were obtained for the different orientations of the polarizer (this corresponds to ellipticities of between $3.5 \%$ and $6 \%$ ). The variability in extinction coefficient (or equivalently in the ellipticity of the light polarization) is likely due to small fluctuation in the minimum signal, which was near the noise threshold. The values of the extinction coefficient that were obtained are consistent with the values expected given the limitations of the EOM, achromatic quarter-wave plate, and Glan-Taylor polarizer used.

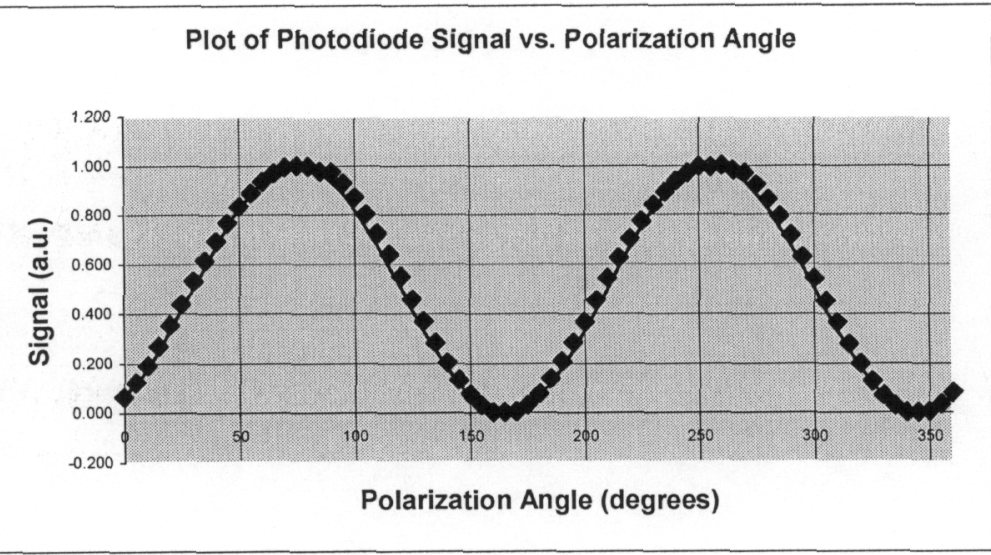

a.) 


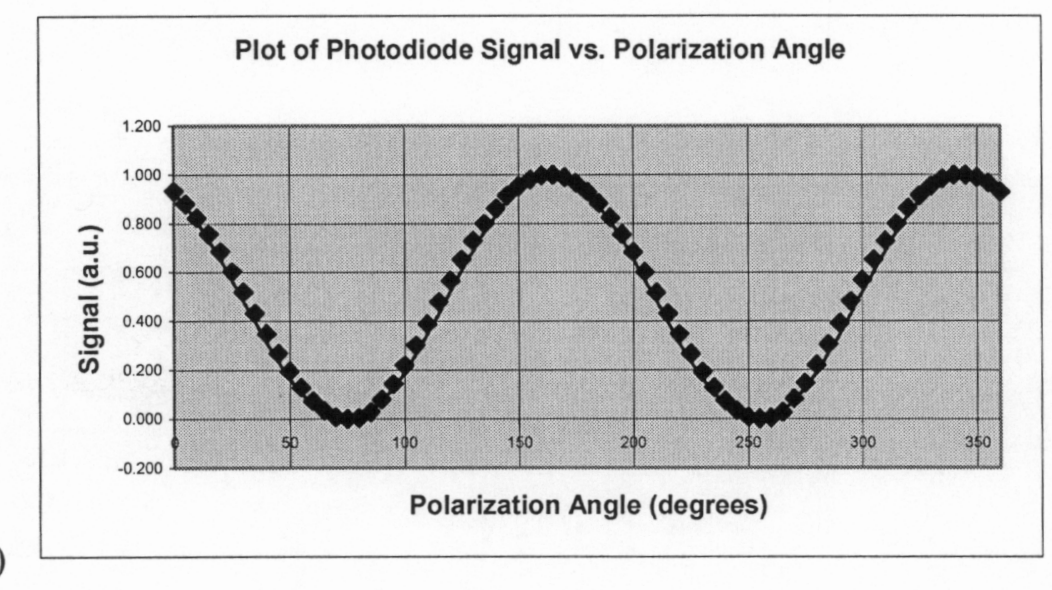

b.)

Figure 5.1. Plot of theoretically fit and measured intensity transmitted through GlanTaylor polarizer as a function of polarization angle. Plots for two different orientations of the Glan-Taylor polarizer ( $a$ and $b$ ) are shown. The theoretical and measured curves were both normalized to 1 .
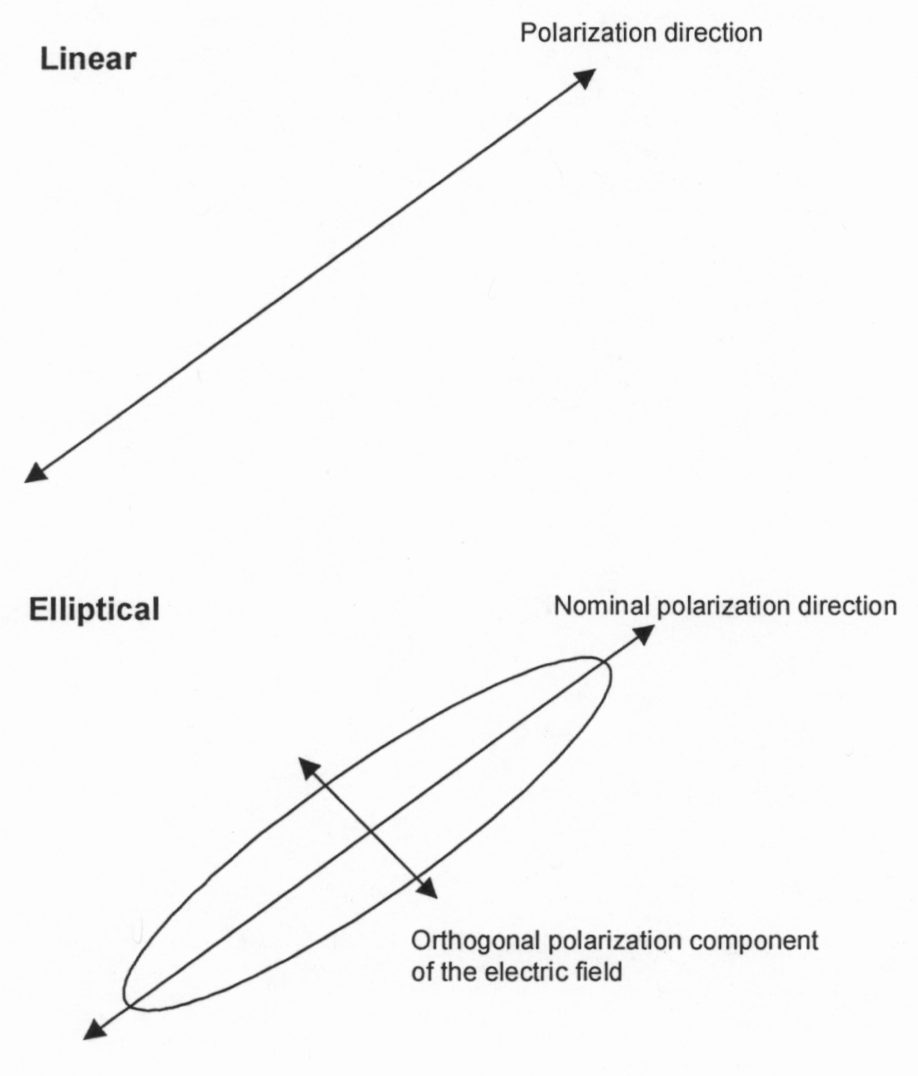

Figure 5.2. Illustration of linearly and elliptically polarized electric field. 
A second test of the system was designed to determine more directly the accuracy of the polarization-modulation technique. This test ensured that there were no irregularities in the input wave - that it was purely sinusoidal. The photodiode signal was measured at the first and second modulation harmonic of the EOM. From Eq. 5.1, the modulated signal is given by $I \propto 1+\operatorname{Cos}(2 \pi \Omega t)$, and thus should only have a component at the first modulation harmonic $(\mathrm{FMH})$ of the EOM. There should be no second harmonic signal at the second modulation harmonic (SMH). The ratio of the signal at the first modulation harmonic to the signal at the second modulation harmonic is given in Table 5.1 for a number of different orientation angles of the Glan-Taylor polarizer. The results again confirm that the polarization of the light is modulated in the manner expected; the fluctuations are likely due to the weak signal at the second modulation harmonic; this signal was near the noise floor. The first and second tests demonstrated that the full-wave voltage of the EOM varies slightly with modulation frequency: The full-wave voltage at $4 \mathrm{kHz}$ (the frequency used for saw-tooth modulation in all experiments) is $1.06 \mathrm{~V}$, while the DC full-wave voltage (EOM voltage at a fixed level) is $1.12 \mathrm{~V}$

Table 5.1. Ratio of photodiode signal at FMH to photodiode signal at SMH

\begin{tabular}{lc}
\hline Polarizer orientation (degrees) & FMH/SMH \\
\hline 0 & 407 \\
\hline 20 & 408 \\
\hline 40 & 507 \\
\hline 60 & 1996 \\
\hline 80 & 674 \\
\hline 100 & 396 \\
\hline 120 & 393 \\
\hline 140 & 1984 \\
\hline 160 & 1000 \\
\hline 180 & 501 \\
\hline
\end{tabular}


Testing the polarization-modulation technique using a quartz waveplate

In order to test the accuracy of the polarization-modulation technique for measuring $R$ and $S$, measurements in a quartz waveplate, with known second-order nonlinear susceptibility, were made. The measurements were designed to test the experimental observations against the predictions of Eq. 3.11. For each microscope objective and collimating lens combination (which resulted in a different numerical aperture for focusing), twenty depth scans ( $1 \mu \mathrm{m}$ resolution) were performed over a 100 to $200 \mu \mathrm{m}$ axial range (depending on the objective) near the front surface of the quartz. The scans were spaced $1 \mu \mathrm{m}$ apart in the transverse direction. The average values (and standard deviations) of $R$ and $S$ obtained in these scans (for data points where the chopper-modulated signal exceeded an arbitrary noise threshold) are summarized in Table 5.2.

Table 5.2. SHG in quartz waveplate for different microscope objectives and collimating lenses

\begin{tabular}{llll}
\hline Microscope obj. & Collimating lens & R: mean \pm std. dev. & S: mean \pm std. dev. \\
\hline $10 \mathrm{X}$ & $\mathrm{f}=100 \mathrm{~mm}$ & $0.243 \pm 0.008$ & $0.94 \pm 0.01$ \\
\hline $10 \mathrm{X}$ & $\mathrm{f}=200 \mathrm{~mm}$ & $0.253 \pm 0.012$ & $0.95 \pm 0.03$ \\
\hline $20 \mathrm{X}$ & $\mathrm{f}=50 \mathrm{~mm}$ & $0.253 \pm 0.007$ & $0.96 \pm 0.01$ \\
\hline $20 \mathrm{X}$ & $\mathrm{f}=100 \mathrm{~mm}$ & $0.255 \pm 0.015$ & $0.92 \pm 0.05$ \\
\hline $20 \mathrm{X}$ & $\mathrm{f}=200 \mathrm{~mm}$ & $0.263 \pm 0.015$ & $0.93 \pm 0.05$ \\
\hline $40 \mathrm{X}$ & $\mathrm{f}=50 \mathrm{~mm}$ & $0.264 \pm 0.015$ & $0.93 \pm 0.04$ \\
\hline $40 \mathrm{X}$ & $\mathrm{f}=100 \mathrm{~mm}$ & $0.265 \pm 0.015$ & $0.93 \pm 0.05$ \\
\hline $40 \mathrm{X}$ & $\mathrm{f}=200 \mathrm{~mm}$ & $0.273 \pm 0.014$ & $0.94 \pm 0.05$ \\
\hline
\end{tabular}

In the quartz waveplate, theory predicts a measurement of $R=0.25$. From Table 5.2 , it is clear that the measurements of $R$ are in very good agreement with the theoretical 
value for all of the objective and collimating lens combinations. Tight focusing can affect the polarization of a collimated beam of light. However, even the tightest focusing used in this experiment (obtained using a $40 \mathrm{X}$ objective and a $200 \mathrm{~mm}$ collimating lens) only introduced a discrepancy of $5 \%$ to $10 \%$.

The quartz crystal also allows correction of the measured value of $S$ for the fact that the chopper modulation is not a perfect sine wave (refer back to Chapter 3 for a more detailed discussion). In quartz, we expect $S_{c o r r}=1.33$ and we measure $S=0.94$ (using the $10 \mathrm{X}$ objective and the $100 \mathrm{~mm}$ collimating lens). Thus, for any sample, the measured value of $S$ should be multiplied by 1.42 in order to obtain the correct ratio of the FMH signal to the DC signal in Eq. 3.8. 


\section{Calibration}

\section{Theory}

The second harmonic signal needs to be calibrated in order to allow measurement of the second order nonlinear susceptibility in tissue samples. A quartz waveplate is used to perform this calibration. The equations describing the second harmonic power produced by a focused Gaussian beam in a uniform material with a second order nonlinear susceptibility (such as quartz) was derived in Chapter 2 (Eqs. 2.11-2.13). For easier reference, these equations are repeated here:

$$
\begin{aligned}
& P_{2 \omega}=\frac{\pi w_{o}^{2}}{2} I_{2 \omega} \\
& I_{2 \omega}=\frac{2 \omega^{2} d_{e f f}^{2} I_{\omega}^{2}\left|J\left(z_{R}, z_{f}, \Delta k\right)\right|^{2}}{n_{1}^{2} n_{2} c^{3} \varepsilon_{o}} \\
& J\left(z_{R}, z_{f}, \Delta k\right)=\int_{-z_{f}}^{-z_{f}+L} \frac{\exp (i \Delta k z)}{1+i z / z_{R}} d z .
\end{aligned}
$$

It is clear from Eqs. 2.11-2.13 that the second harmonic signal depends strongly on the focal spot Rayleigh range $z_{R}$ (which is directly related to the focal spot size $w_{o}$ by the expression $z_{R}=\frac{n, \pi w_{0}^{2}}{\lambda}$ ). Therefore, even if input laser power is held constant, SHG signal measurements made using objective and collimating lens combinations with different numerical apertures for focusing the beam cannot be compared directly. In order to make useful comparison, information about $z_{R}$ for each of the different numerical apertures used must be obtained. While it is possible in theory to calculate the effective 
numerical aperture of a given laser beam profile, spatial filter, and focusing objective, this is very difficult to do accurately in practice. Instead, measurements of the second harmonic signal in quartz, which has a known second order nonlinear susceptibility, can be used to determine $z_{R}$. Using quartz to calibrate the experimental setup has the added advantage of allowing the power of the second harmonic signal generated in collagen to be determined.

The same $\sim 700 \mu \mathrm{m}$ thick quartz waveplate that was used to confirm the accuracy of the polarization modulation technique is used as the reference standard. The optic axis of the waveplate is in the plane of the waveplate and the laser beam propagates normal to this plane; Figure 3a illustrates this geometry. Eqs. 2.11-2.13 above can also be used to describe second harmonic generation in the quartz waveplate as a function of focal depth. Figure $3 \mathrm{~b}$ shows several examples of the theoretical depth dependence of SHG in quartz for different values of $z_{R}$. There is a unique relationship between the full width at half maximum of the peaks in signal at the two surfaces of the waveplate and $z_{R}$. Therefore, the quartz waveplate can be used to directly determine $z_{R}$ for different microscope objectives and collimating lenses. 
a.)
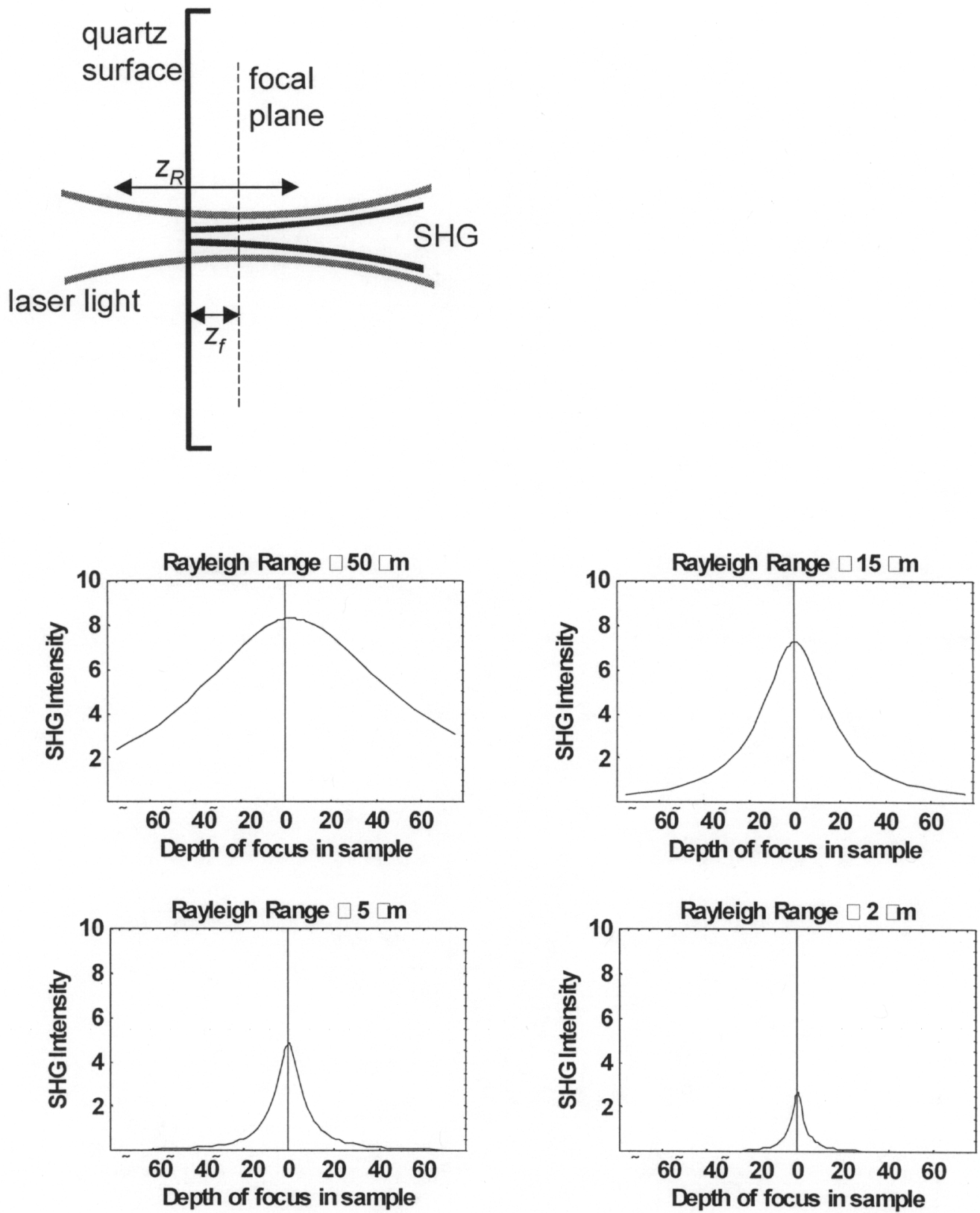

b.)

Figure 5.3. (a.) Illustration of SHG measurement in quartz. (b.) Theoretical calculation of second harmonic signal as a function of depth of focus (depth in sample increases with increasing $\mathrm{z}$ ) generated in a quartz waveplate $(\mathrm{z}=0$ is the air-quartz interface) by beams focused with different Rayleigh ranges.

The second harmonic signal is peaked at the surfaces of the waveplate. This effect is due to the Gouy phase shift: the phase of the first and second harmonic light are 
both shifted as they pass through the focus, but, because the second harmonic light is at half the wavelength of the fundamental beam, it is shifted by twice as much. The fact that the fundamental beam and the second harmonic beam rapidly go out of phase in a tight focus leads to cancellation of the second harmonic light in the bulk of uniform samples, such as a quartz crystal. A large SHG signal occurs only when the beam is focused at the front or back surface of the quartz. A related phenomenon in third harmonic generation microscopy has been used to obtain information about the dispersion and nonlinear susceptibilities of materials at interfaces (Barad et. al., 1997; Schins et. al., 2002).

The theory outlined in Eqs. 2.11-2.13 makes the paraxial approximation, which assumes that the polarization of the laser light is unchanged in the focus. This is not strictly true, especially for the case of tight focusing (high numerical aperture). The theory predicts a quantitative scaling for the SHG signal as a function of the beam parameters of the focused beam. From Eqs. 2.11 and 2.12, we see that

$$
P_{2 \omega} \propto\left|J\left(z_{R}, z_{f}, \Delta k\right)\right|^{2} / w_{o}^{2},
$$

where we use the peak value of $|J|^{2}$ as a function of focal depth $\left(z_{f}=z_{f \max } \sim 0\right)$. To test how well the theory outlined in Eqs. 2.11-2.13 applies to the measurements, we compare the peak second harmonic signal obtained using different microscope objectives and collimating lenses. The peak signal should be related to the spot radius through Eq. 5.2. We estimate the spot radius indirectly by determining the Rayleigh range $z_{R}$, assuming a Gaussian beam. Our measurements (see below) suggest that the scaling in Eq. 5.2 holds 
for our beam and the focusing conditions. This gives us confidence that we can use the quartz signal to calibrate the setup. By referencing the collagen signal against the quartz standard, we can determine the magnitude of the elements of the nonlinear susceptibility tensor. Assuming the same laser input power and spot size, then, for a given ratio of the signal in collagen $V_{\text {collagen }}$ to the signal in quartz $V_{\text {quartz }}$ we obtain,

$$
d_{\text {eff (collagen) }}^{2}=\frac{V_{\text {collagen }}\left|J_{\text {quarz }}\left(z_{R}, z_{f \max }, \Delta k\right)\right|^{2}}{V_{\text {quartz }}\left|J_{\text {collagen }}\left(z_{R}, z_{f \max }, \Delta k\right)\right|^{2}} d_{\text {eff (quart) }}^{2}
$$

In quartz, the ordinary index of refraction at the laser frequency is $n_{l}=1.5383$ and the ordinary index of refraction at the second harmonic frequency is $n_{2}=1.5577$ (Ghosh, 1999). The index of refraction in collagen is approximately 1.5 , so we have assumed in obtaining Eq. 5.3 that the indices in collagen and quartz are equal where they appear in Eq. 2.12. Note that we must take into account the difference in $\Delta k$ between collagen and quartz because the calculation of $|J|^{2}$ is sensitive to it. 


\section{Measurement}

The second harmonic signal in a quartz waveplate was measured in order to establish it as a reference standard. As noted above we need to measure more than the peak second harmonic signal in quartz; we must also measure the Rayleigh range $z_{R}$ of the focal spot. For each microscope objective and collimating lens combination, twenty depth scans ( $1 \mu \mathrm{m}$ resolution) over a 100 to $200 \mu \mathrm{m}$ axial range (depending on the objective) near the front surface of the quartz. The scans were spaced $1 \mu \mathrm{m}$ apart in the transverse direction. The results were averaged to obtain a curve of the second harmonic signal as a function of depth, such as the example plotted in Figure 5.3. Because spherical aberration at the quartz interface affects the peak SHG signal when the focus is at the back surface of the quartz waveplate, we only use results obtained from the front surface. All of these results were obtained using an incident average laser power of $15.0 \mathrm{~mW}$ (measured before the microscope objective). We are interested in the magnitude (maximum signal) and shape (full-width at half-maximum) of the peak in SHG signal that occurs at the front surface of the quartz waveplate; the results for all of the microscope objective and collimating lens combinations are summarized in Table 5.3. 


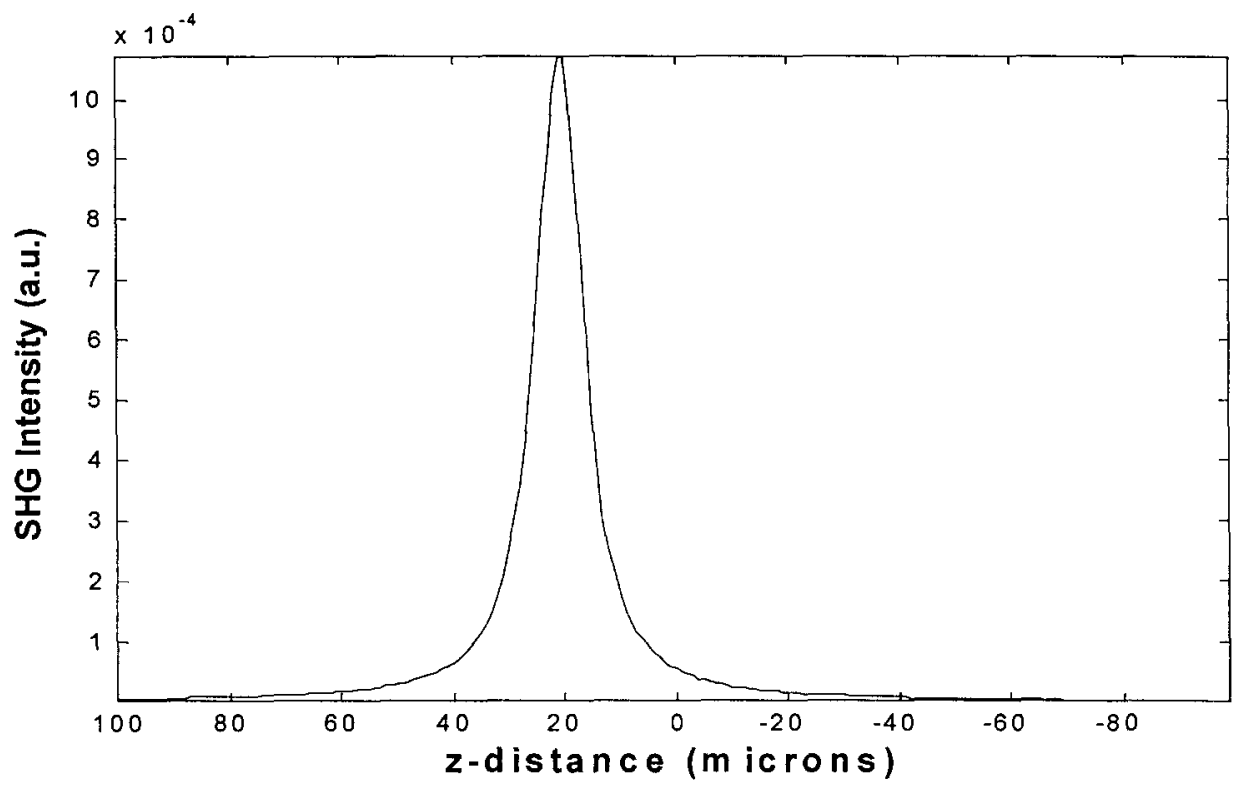

Figure 5.4. Second harmonic signal intensity as a function of depth in quartz near top surface (increasing $\mathrm{z}$ corresponds to increasing depth in the sample) using the $20 \mathrm{X}$ objective and a $1 \mu \mathrm{m}$ scan resolution (arbitrary origin).

Table 5.3. SHG in quartz waveplate for different microscope objective and collimating lens combinations

\begin{tabular}{llll}
\hline $\begin{array}{l}\text { Microscope } \\
\text { Objective }\end{array}$ & Collimating lens & $\begin{array}{l}\text { Peak SHG signal }(\mathbf{m V}) \\
(\mathbf{1 5} \mathbf{~ m W} \text { incident power) }\end{array}$ & $\begin{array}{l}\text { Full-width at half- } \\
\text { maximum of } \\
\text { signal peak }\end{array}$ \\
\hline $10 \mathrm{X}$ & $\mathrm{f}=100 \mathrm{~mm}$ & 0.19 & $130.1 \mu \mathrm{m}$ \\
\hline $10 \mathrm{X}$ & $\mathrm{f}=200 \mathrm{~mm}$ & 0.54 & $35.0 \mu \mathrm{m}$ \\
\hline $20 \mathrm{X}$ & $\mathrm{f}=50 \mathrm{~mm}$ & 0.32 & $61.1 \mu \mathrm{m}$ \\
\hline $20 \mathrm{X}$ & $\mathrm{f}=100 \mathrm{~mm}$ & 0.54 & $29.6 \mu \mathrm{m}$ \\
\hline $20 \mathrm{X}$ & $\mathrm{f}=200 \mathrm{~mm}$ & 1.07 & $10.5 \mu \mathrm{m}$ \\
\hline $40 \mathrm{X}$ & $\mathrm{f}=50 \mathrm{~mm}$ & 0.89 & $16.5 \mu \mathrm{m}$ \\
\hline $40 \mathrm{X}$ & $\mathrm{f}=100 \mathrm{~mm}$ & 1.26 & $10.0 \mu \mathrm{m}$ \\
\hline $40 \mathrm{X}$ & $\mathrm{f}=200 \mathrm{~mm}$ & 0.74 & $6.1 \mu \mathrm{m}$ \\
\hline
\end{tabular}

The width of the second harmonic peak at the front surface of the quartz waveplate is related to the Rayleigh range of the focused beam. It is possible to calculate the Rayleigh range from the full width at half maximum (FWHM) of the measured second harmonic peak. In Table 5.4 the Rayleigh range $z_{R}$, spot size $w_{o}$, numerical 
aperture NA, $|J|^{2}$, and $|J|^{2} / w_{o}^{2}$ calculated from the measured FWHM for each microscope objective is given (the focal length of the beam expander collimating lens is given in parentheses next to the microscope objective).

Table 5.4. SHG in quartz, determining $z_{R}, w_{o}$, and $|J|^{2}$

\begin{tabular}{lcccccc}
\hline $\begin{array}{l}\text { Microscope } \\
\text { Objective }\end{array}$ & FWHM & $\mathbf{z}_{\mathbf{R}}(\mu \mathbf{m})$ & $\mathbf{w}_{\mathbf{0}}(\mu \mathbf{m})$ & $\mathbf{N A}$ & $|\mathbf{J}|^{2}\left(\mu \mathbf{m}^{2}\right)$ & $|J|^{2} / w_{o}^{2}$ \\
\hline $10 \mathrm{X}(100 \mathrm{~mm})$ & 130.1 & 60.7 & 3.9 & 0.13 & 11.4 & 0.7 \\
\hline $10 \mathrm{X}(200 \mathrm{~mm})$ & 35.0 & 15.0 & 2.0 & 0.24 & 8.0 & 2.1 \\
\hline $20 \mathrm{X}(50 \mathrm{~mm})$ & 61.1 & 27.7 & 2.7 & 0.18 & 9.5 & 1.3 \\
\hline $20 \mathrm{X}(100 \mathrm{~mm})$ & 29.6 & 12.5 & 1.8 & 0.27 & 7.5 & 2.4 \\
\hline $20 \mathrm{X}(200 \mathrm{~mm})$ & 10.5 & 3.7 & 1.0 & 0.49 & 4.2 & 4.5 \\
\hline $40 \mathrm{X}(50 \mathrm{~mm})$ & 16.5 & 6.3 & 1.3 & 0.38 & 5.7 & 3.5 \\
\hline $40 \mathrm{X}(100 \mathrm{~mm})$ & 10.0 & 3.5 & 0.9 & 0.52 & 4.0 & 4.6 \\
\hline $40 \mathrm{X}(200 \mathrm{~mm})$ & 6.1 & 1.9 & 0.7 & 0.85 & 2.6 & $3.3^{*}$ \\
\hline
\end{tabular}

Because the 100X objective (NA $=0.7)$ used to collect the second harmonic light does not actually collect all of the light generated by the $40 \mathrm{X}$ objective $(\mathrm{NA}=0.9)$ this value has been multiplied by a correction factor of 0.61 .

Eq. 5.2 predicts a scaling relation for $\mathrm{SHG}$ as a function of $w_{o}$. In Figure 5.4 the ratio of the theoretical peak signal (normalized to the peak signal calculated for the $10 \mathrm{X}$ objective and $100 \mathrm{~mm}$ collimating lens) to the measured peak signal (also normalized) versus the numerical aperture of each combination of objective and collimating lens we used is plotted. If the predicted scaling is satisfied, then all of the points plotted should fall on a line of slope 1 (plotted as a thick diagonal line in Figure 5.4). Figure 5.4 shows that there is reasonable agreement between the measured and theoretical ratios of the SHG signal from the different objectives. This holds true even for numerical apertures larger than 0.4, where the paraxial approximation using in the calculations begins to break down. 


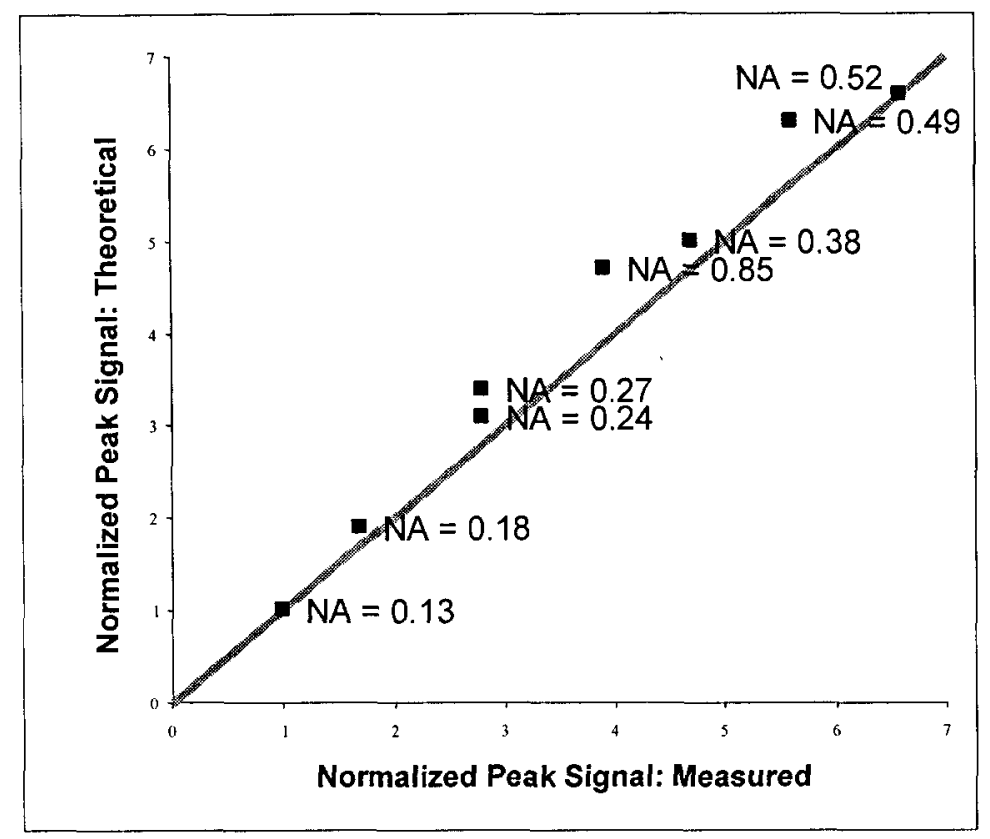

Figure 5.5. Plot of the theoretically calculated peak signal (normalized to the value calculated for the $10 \mathrm{X}$ objective and $100 \mathrm{~mm}$ collimating lens) versus the measured peak signal (normalized to the value measured for the $10 \mathrm{X}$ objective and $100 \mathrm{~mm}$ collimating lens). The squares are the data points; the gray line (slope of 1) represents the value expected for perfect agreement between theory and measurement.

There are several factors that can explain the discrepancies (of up to 20\%) between theory and experiment. The theory assumes a Gaussian beam; it neglects the fact that the beam incident on the back aperture of the objective is the central maximum of the pattern produced by the spatial filter. The theory also does not take into account the fact that the polarization of tightly focused beams is not simply normal to the propagation direction but contains additional components. Researchers who previously investigated the qualitative effect of changing the numerical aperture on SHG microscopy (Gauderon et. al., 2001) also noted that differing group velocity dispersion in different microscope objectives may play a role. 


\section{References}

Y. Barad, H. Eisenberg, M. Horowitz, and Y. Silberberg. 1997. Nonlinear scanning laser microscopy by third harmonic generation. Applied Physics Letters. 70:922-924.

R. Gauderon, P. B. Lukins, C. J. R. Sheppard. 2001. Optimization of second-harmonic generation microscopy. Micron. 32:691-700.

K. König, O. Krauss, and I. Riemann. 2002. Intratissue surgery with $80 \mathrm{MHz}$ nanojoule femtosecond laser pulses in the near infrared. Optics Express. 10: 171-176.

J. Noack and A. Vogel. 1999. Laser-induced plasma formation in water at nanosecond to femtosecond time scales: Calculation of thresholds, absorption coefficients, and energy density. IEEE Journal of Quantum Electronics. 35:1156-1167.

A. M. Oravesky, L. B. Da Silva, A. M. Rubenchik, M. D. Feit, M. E. Glinsky, M. D. Perry, B. M. Mammini, W. Small, IV, and B. C. Stuart. 1996. Plasma mediated ablation of biological tissues with nanosecond-to-femtosecond laser pulses: Relative role of linear and nonlinear absorption. IEEE Journal of Selected Topics in Quantum Electronics. 2:801-809.

C. B. Schaffer, A. Brodeur, and E. Mazur. 2001. Laser-induced breakdown and damage in bulk transparent materials induced by tightly focused femtosecond laser pulses. Measurement Science and Technology. 12:1784-1794.

J. M. Schins, G. J. Brakenhoff, M. Müller. 2002. Characterizing layered structures with third-harmonic generation microscopy. G. I. T. Imaging \& Microscopy. 1:44-46.

B. C. Stuart, M. D. Feit, S. Herman, A. M. Rubenchik, B. W. Shore, and M. D. Perry. 1996. Nanosecond-to-femtosecond laser-induced breakdown in dielectrics. Physical Review B. 53:1749-1761. 


\section{THE MAGNITUDE OF THE SECOND HARMONIC SIGNAL IN COLLAGEN}

In this chapter, we focus on inferences that may be drawn from measuring the magnitude of the second harmonic signal; the polarization dependence of the second harmonic signal is the subject of the two subsequent chapters. Specifically, this chapter deals with the observed heterogeneity of second harmonic signal magnitude that is structurally homogeneous under a polarized light microscope. SHG microscopy images of thin sections of rat-tail tendon show that, even in unmodified samples of this highly ordered tissue with collagen fibrils aligned in the plane normal to the laser beam, the second harmonic signal varies substantially (by one to two orders of magnitude) with transverse position in the plane of the sample. What could lead to the variability in second harmonic signal? One possible explanation is that collagen fibrils are randomly oriented parallel and anti-parallel to their neighbors, as was inferred from electron microscopy studies (Parry and Craig, 1977). As discussed in Chapter 2, random parallel and anti-parallel orientation of the fibrils would lead to constructive and destructive interference, respectively, of the second harmonic light in different regions of the tissue (Figure 6.1). Using microscope objectives of different numerical apertures, we explore this possibility and quantify the distance over which neighboring collagen fibrils are likely to be oriented parallel to each other. 

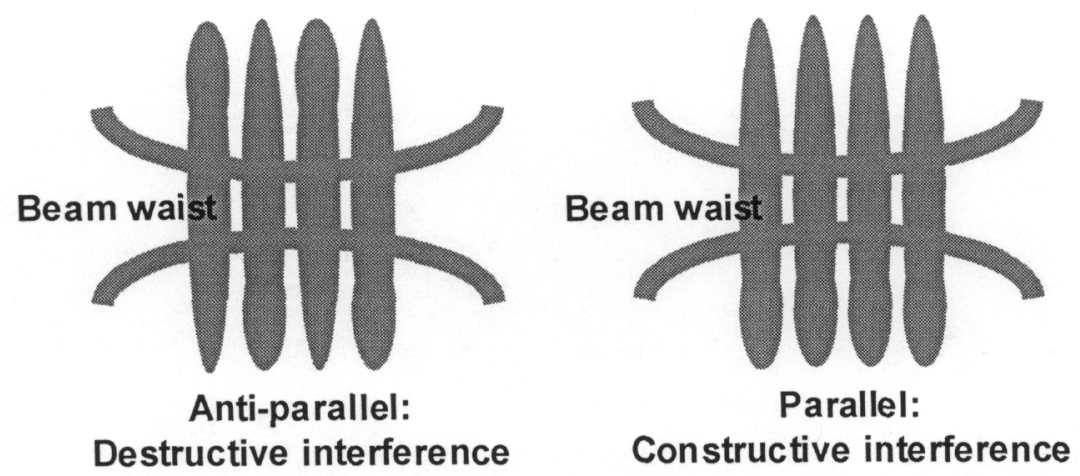

Figure 6.1. Sketch of parallel and anti-parallel orientation of collagen fibrils. (a.) Region with collagen fibrils oriented parallel. (b.) Region with collagen fibrils oriented antiparallel.

In addition to providing information about the parallel and anti-parallel orientation of neighboring collagen fibrils, the magnitude of the second harmonic signal yields information about the second order nonlinear susceptibility tensor-namely the magnitude of its elements (again, refer to Chapter 2). By calibrating the experimental setup using a quartz waveplate, a lower bound can be placed on the second-order nonlinear susceptibility of collagen.

\section{Tissue source}

Type I collagen fascicles were harvested from tails of 3-4 month old SpragueDawley rats that had been stored at -20 degrees $\mathrm{C}$. Individual tendon fascicles were removed from the tendon bundles under a dissecting microscope. Typical fascicles were several centimeters long, and had a diameter of only a few tenths of a millimeter. Some samples were simply placed on glass slides beneath a cover slip. Other samples were prepared for analysis as frozen sections; $4 \mu \mathrm{m}$ serial slices were obtained from 
approximately $20 \mu \mathrm{m}$ to $30 \mu \mathrm{m}$ below the surface. The unstained sections were mounted on glass slides beneath a cover slip.

\section{Second harmonic signal magnitude in intact tendon fascicles}

Two SHG microscopy images of regions of a rat-tail tendon fascicle collected in transmission mode are presented in Figure 6.2. Figure 6.2 shows a transverse scan of the fascicle; both the $\mathrm{x}$-axis and $\mathrm{y}$-axis are in the plane of the fascicle, normal to the laser propagation direction (Figure 6.3). The image in Figure 6.2 was obtained using the $20 \mathrm{X}$ objective, the $200 \mathrm{~mm}$ collimating lens, and a scan resolution of $1 \mu \mathrm{m}$; the signal was detected at the chopper modulation frequency. Note the strong variability in the SHG signal. An image was obtained simultaneously in reflection mode (Figure 6.4).

As discussed in Chapter 2, we would expect the second harmonic signal generated in the forward direction to be weaker in regions of anti-parallel collagen fibril alignment and stronger in regions of parallel alignment. There would be little effect on the second harmonic signal generated in the back-scattering direction. However, a significant fraction of the back-scattered second harmonic light is probably reflected at boundaries between the sample, the glass slide, and the air. In addition, the backscattering efficiency within the tissue may change with position. Thus, it is difficult to interpret any differences between the transmission mode and reflection mode images as the effect of the parallel and anti-parallel alignment of neighboring fibrils. Therefore, in the following section, we turn to a quantitative technique that uses thin sections of tissue and transmission mode detection to study the variation in second harmonic signal strength. 


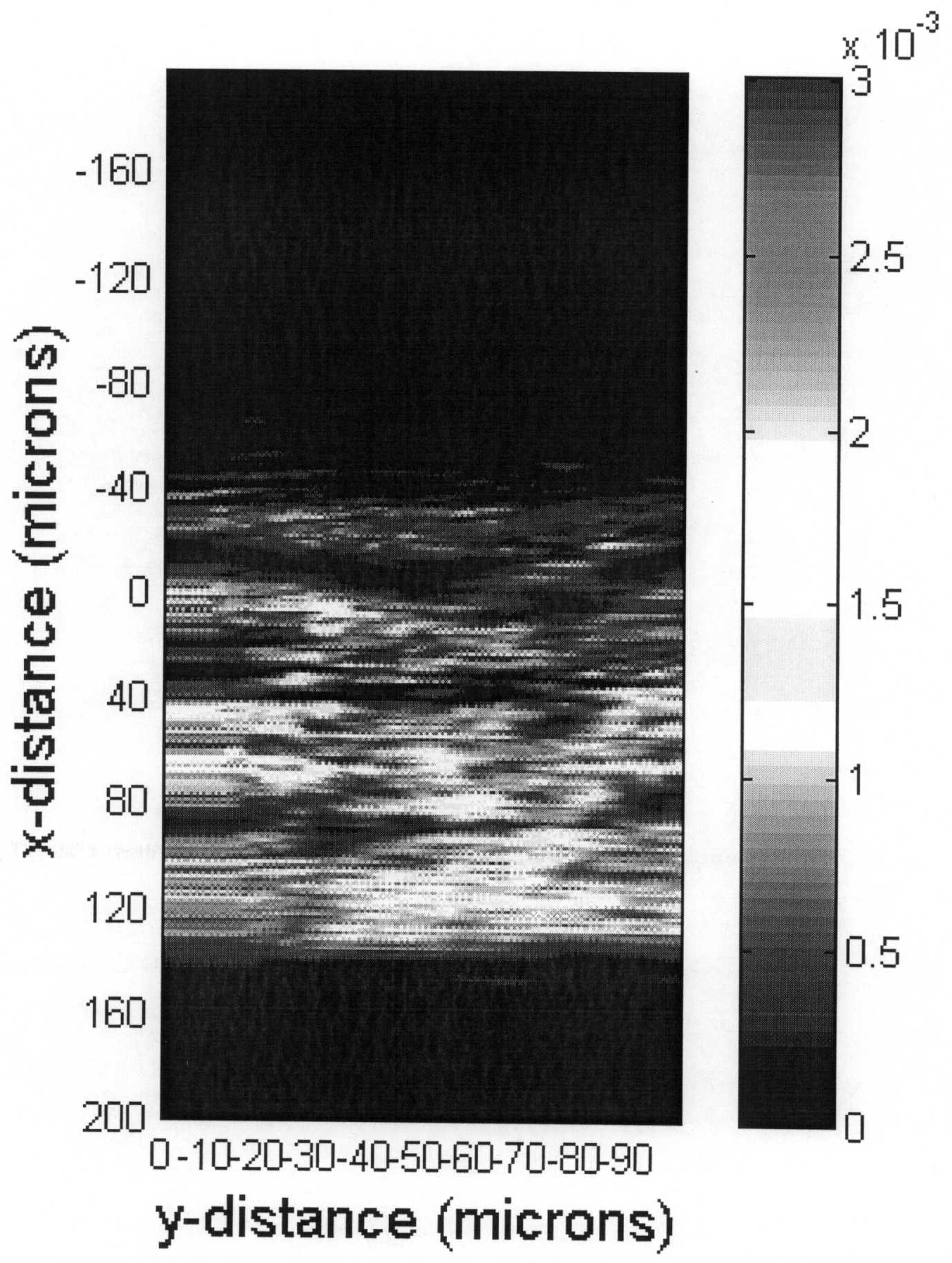

Figure 6.2. Transmission mode SHG microscopy. The color scale indicates signal strength at the chopper frequency in arbitrary units. Image of an $x-y$ cross-section of a rat-tail tendon fascicle, obtained near the center (in depth) of the fascicle. 


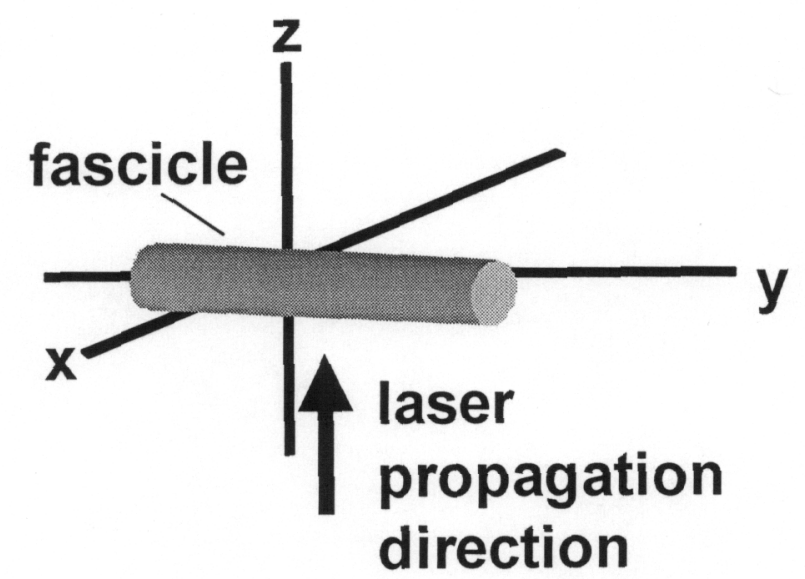

Figure 6.3. Sketch of fiber orientation and beam propagation direction and polarization. 


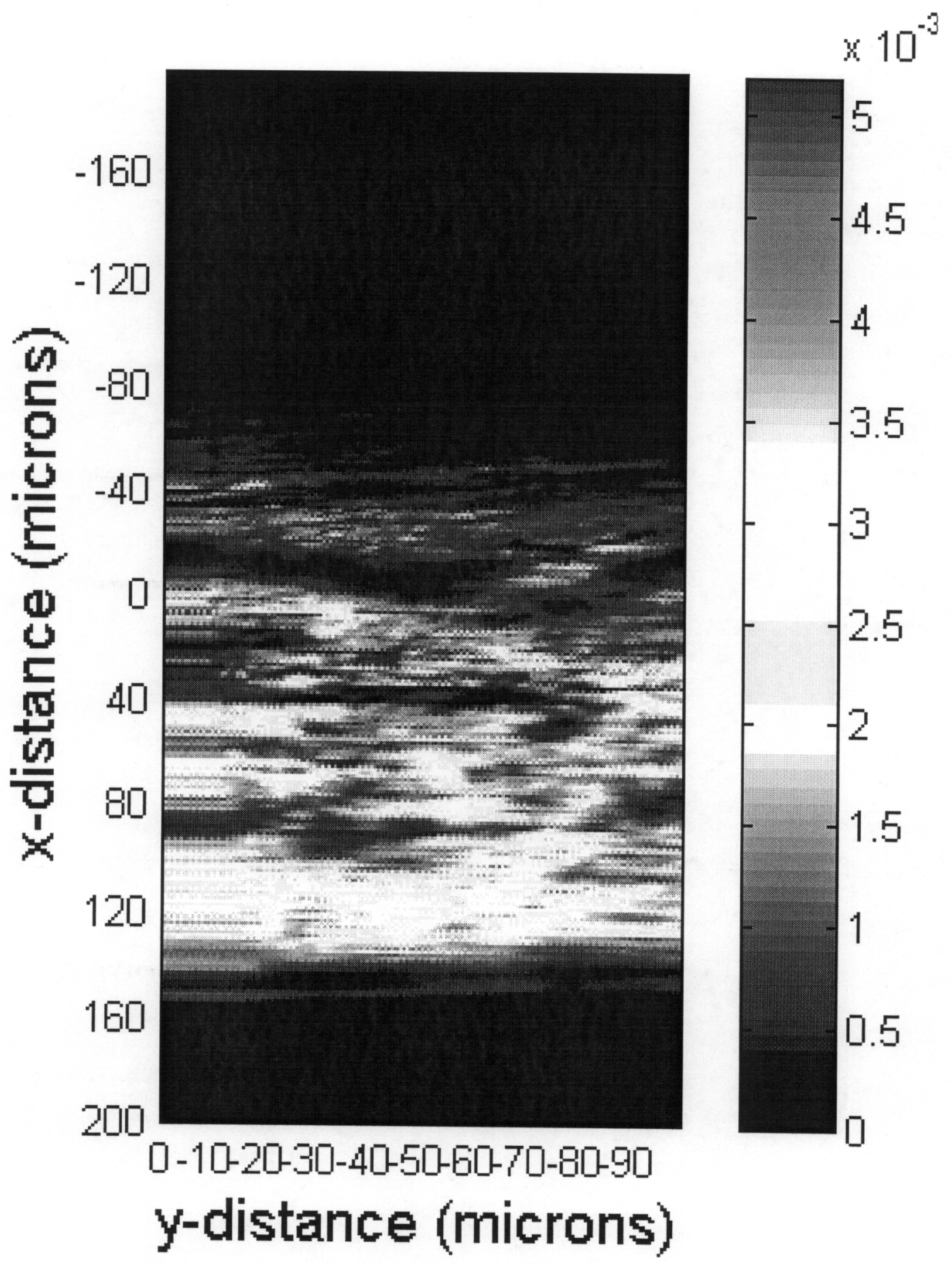

Figure 6.4. Reflection mode SHG microscopy. The color scale indicates signal strength at the chopper frequency in arbitrary units. Image of an $x-y$ cross-section of a rat-tail tendon fascicle, obtained near the center (in depth) of the fascicle. 


\section{Second harmonic signal magnitude in tendon sections}

To measure the variability in SHG signal quantitatively, the signal was measured in thin sections of the same tissue. In these sections, possible confounding effects-such as variability in the sample thickness, birefringence, and scattering-are eliminated. For each microscope objective and collimating lens combination the second harmonic signal in a $100 \mu \mathrm{m}$ by $100 \mu \mathrm{m}$ region of a rat-tail tendon frozen section was measured using a 1 $\mu \mathrm{m}$ scan resolution. The resulting images are plotted in Figure 6.5 (the signal at the chopper modulation frequency- $\mathrm{ZMH}$ - is plotted); no smoothing has been applied in order to illustrate the difference in resolution. The intensity measurements obtained clearly fluctuate over a broad range between the noise floor and the maximum signal in all of the images. There is also a large variation in SHG signal between the different images (peak signal strength generally increases with tighter focusing and higher resolution); note that each image in Figure 6.5 uses a different color scale.

a.)

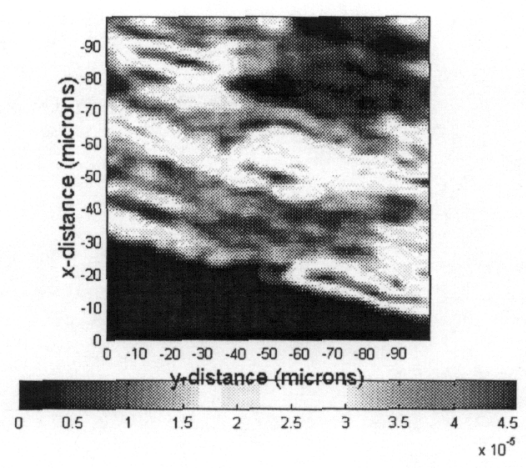

b.)

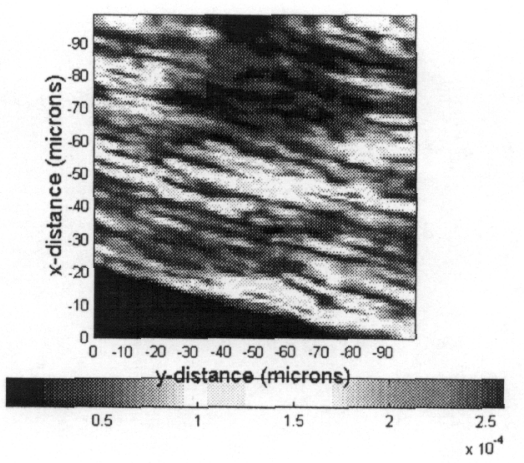


c.)

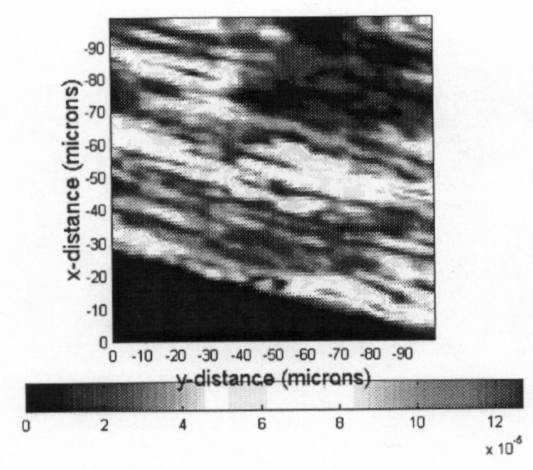

e.)

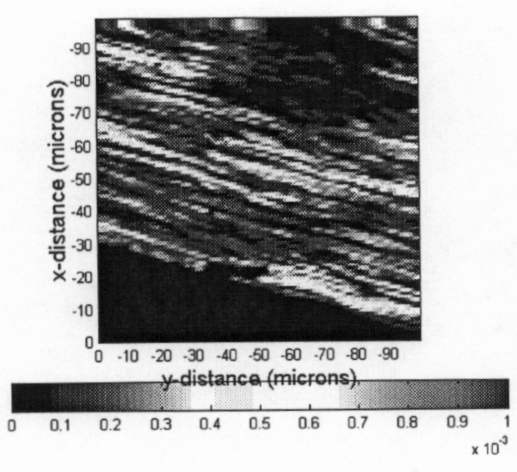

g.)

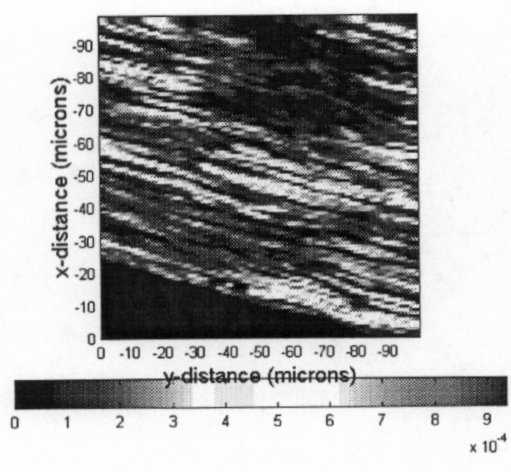

d.)

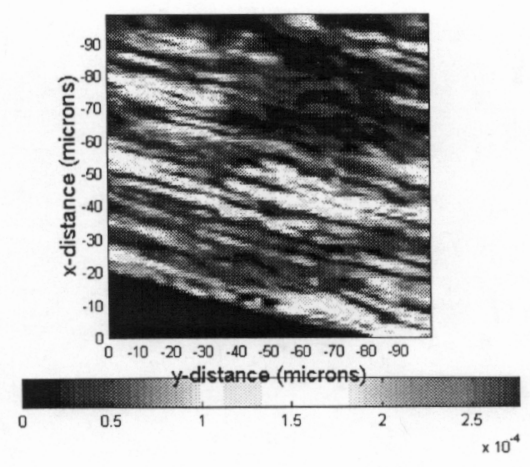

f.)

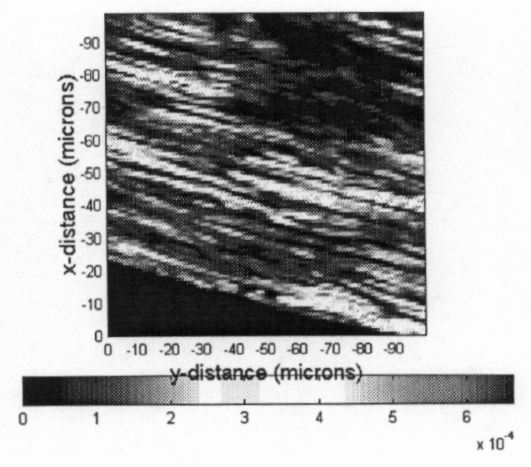

h.)

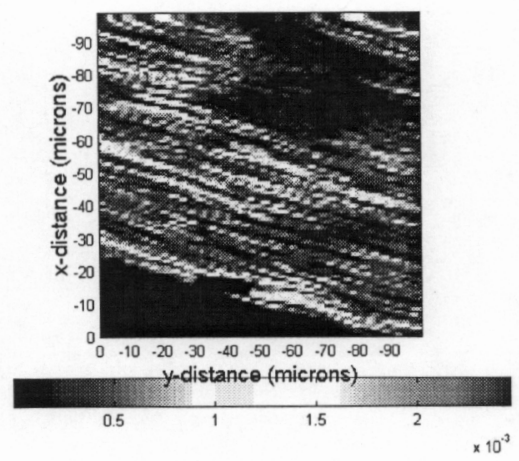

Figure 6.5. Second harmonic signal as a function of transverse position in a section of rat-tail tendon obtained using a $1 \mu \mathrm{m}$ scan resolution and the (a) $10 \mathrm{X}$ objective with the $100 \mathrm{~mm}$ collimating lens, (b) $10 \mathrm{X}$ objective with the $200 \mathrm{~mm}$ collimating lens, (c) $20 \mathrm{X}$ objective with the $50 \mathrm{~mm}$ collimating lens, (d) $20 \mathrm{X}$ objective with the $100 \mathrm{~mm}$ collimating lens, (e) $20 \mathrm{X}$ objective with the $100 \mathrm{~mm}$ collimating lens, (f) $40 \mathrm{X}$ objective with the $50 \mathrm{~mm}$ collimating lens, (g) $40 \mathrm{X}$ objective with the $100 \mathrm{~mm}$ collimating lens, and (h) $40 \mathrm{X}$ objective with the $200 \mathrm{~mm}$ collimating lens. The sharp edges in the higher resolution images are due to the finite pixel size; the data are not smoothed. 
Two possibilities could lead to the large variation in signal strength as a function of transverse position. One possibility is the existence of narrow, localized bands of much higher collagen density are responsible; another possibility is that the parallel and anti-parallel orientation of neighboring collagen fibrils is responsible. No studies in the literature that we are aware of report on any significant variation in density with position in rat-tail tendon (refer, for instance, to Baer et. al., 1988). Furthermore, a comparison of two-photon fluorescence and SHG images (obtained using an excitation wavelength of $740 \mathrm{~nm}$ ) shows that the two photon fluorescence signal varies much less than the second harmonic signal (Figure 6.6). Two photon fluorescence is an incoherent process that is independent of the parallel or anti-parallel orientation of neighboring collagen fibrils. Therefore, a likely explanation for the variability in second harmonic signal is that there are regions over which neighboring fibrils have the same polarity, leading to coherent enhancement of the SHG signal, and other regions with neighboring fibrils having random polarity, leading to cancellation of the SHG signal (see Chapter 2).
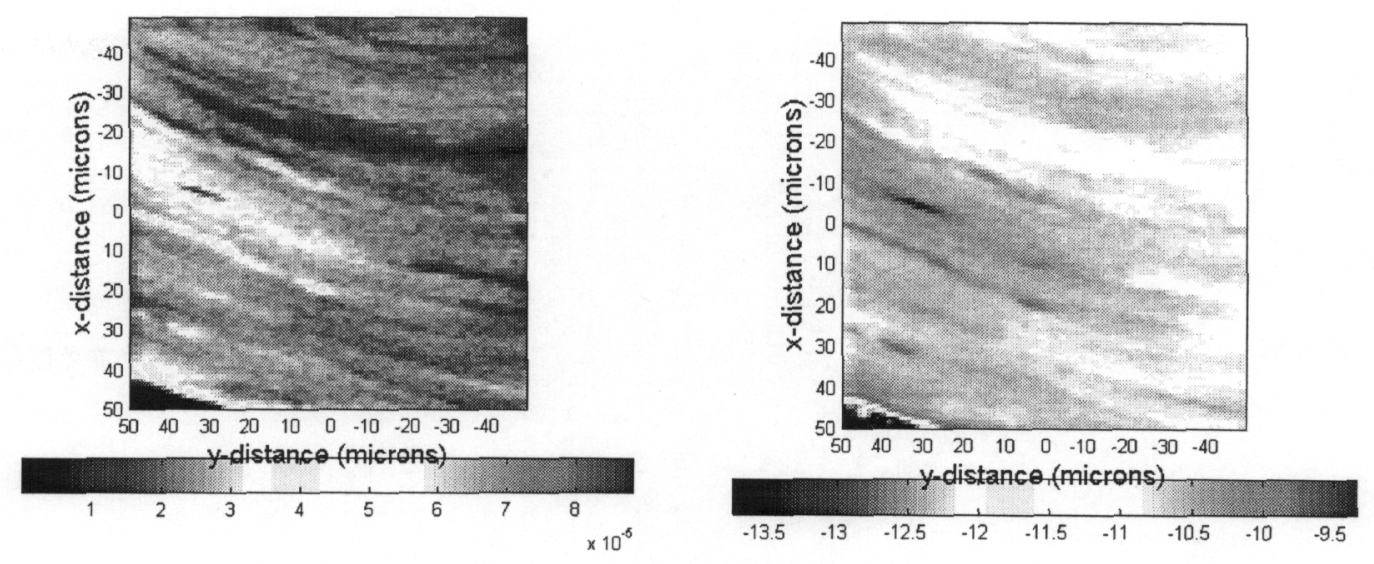

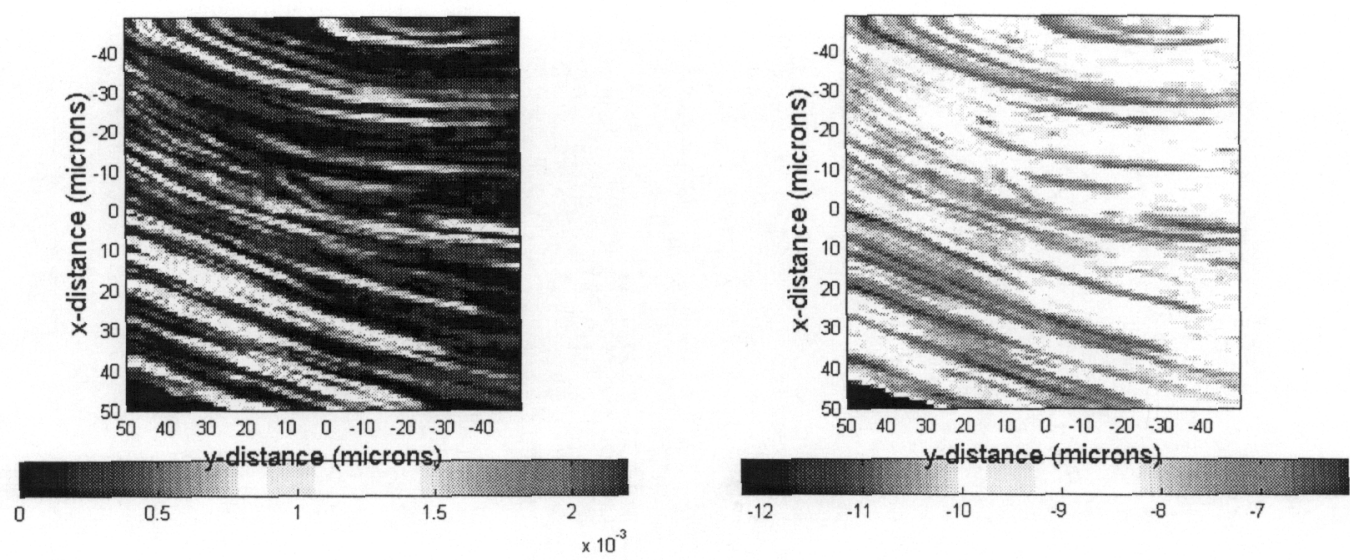

Figure 6.6. Linear scale (a) and logarithmic scale (b) images of two photon fluorescence signal in a section of rat-tail tendon fascicle. Linear scale (c) and logarithmic scale (d) images of second harmonic signal in the same region of tissue as in (a) and (b).

The local variation of collagen fibril polarity is poorly understood. Previous electron microscopy studies (Parry and Craig, 1977) indicate that collagen fibrils have random polarity with respect to their neighbors, while previous sum frequency generation experiments (Freund et. al., 1986) suggest that there are regions of fibrils with the same polarity. But on what scale do fluctuations in polarity occur? Freund et. al. (1986) concluded that these fluctuation occur over regions less than $5 \mu \mathrm{m}$ in width, the resolution of their crossed beam sum frequency generation experiment. The second harmonic images of sections of rat-tail tendon in Figure 6.5 indicate that regions of peak signal-probably corresponding to regions where most of the fibrils have the same polarity-are very narrow in the direction normal to the fibrils. Even when the $40 \mathrm{X}$ objective (with the $200 \mathrm{~mm}$ collimating lens) is used, these regions are not completely resolved, indicating that they are less than $\sim 1.5 \mu \mathrm{m}$ (the optical resolution) in width. The small size of these regions suggests that several large diameter fibrils $(\sim 0.5 \mu \mathrm{m})$ with the same polarity could be responsible for the enhanced SHG signal (Parry and Craig, 1977). 
This would be consistent with both the generally random fibril orientation observed in electron microscopy studies and with the observations of Freund et. al. (1986).

Another approach to analyzing the variation in signal strength is to compare the distribution of the second harmonic signal strength observed using different numerical apertures. Figure $6.7 \mathrm{a}$ is a $\log -\log$ plot of the number of points with a given SHG signal strength versus the signal strength. Clearly, signal strength increases with increasing numerical aperture, but this is mainly because the tighter focusing allowed by a higher numerical aperture increases the intensity of the laser light in the focus. The SHG data for each image in Figure 6.5 can be normalized by removing the $1 / w_{o}^{2}$ scaling expected from Eq. 5.2. This normalization serves to reveal effects associated with averaging over a finite spot size. Figure $6.7 \mathrm{~b}$ shows a $\log -\log$ plot of the number of points with normalized SHG signal strength versus the normalized signal strength for each microscope objective and collimating lens used. The numerical aperture measured using the quartz wave-plate $\left(N A_{\text {meas }}\right)$ is given as a reference for each curve. We see from Figure $6.7 \mathrm{~b}$ that there is little difference in the measured signal (after correction for higher intensity with tighter focusing) for the different numerical apertures. Only use of the highest numerical aperture (corresponding to a focal spot diameter of $\sim 1.5 \mu \mathrm{m}$ ) leads to several outlying points with a significantly stronger normalized signal than was observed with the other numerical apertures. This suggests that the polarity of neighboring fibrils becomes non-random only on scales comparable to or smaller than the $\sim 1.5 \mu \mathrm{m}$ spot diameter formed by the highest numerical aperture objective. Furthermore, this suggests that the polarity of collagen fibrils is random on scales ranging from $\sim 2 \mu \mathrm{m}$ to $\sim 8 \mu \mathrm{m}$ - the range of focal spot diameters used. 

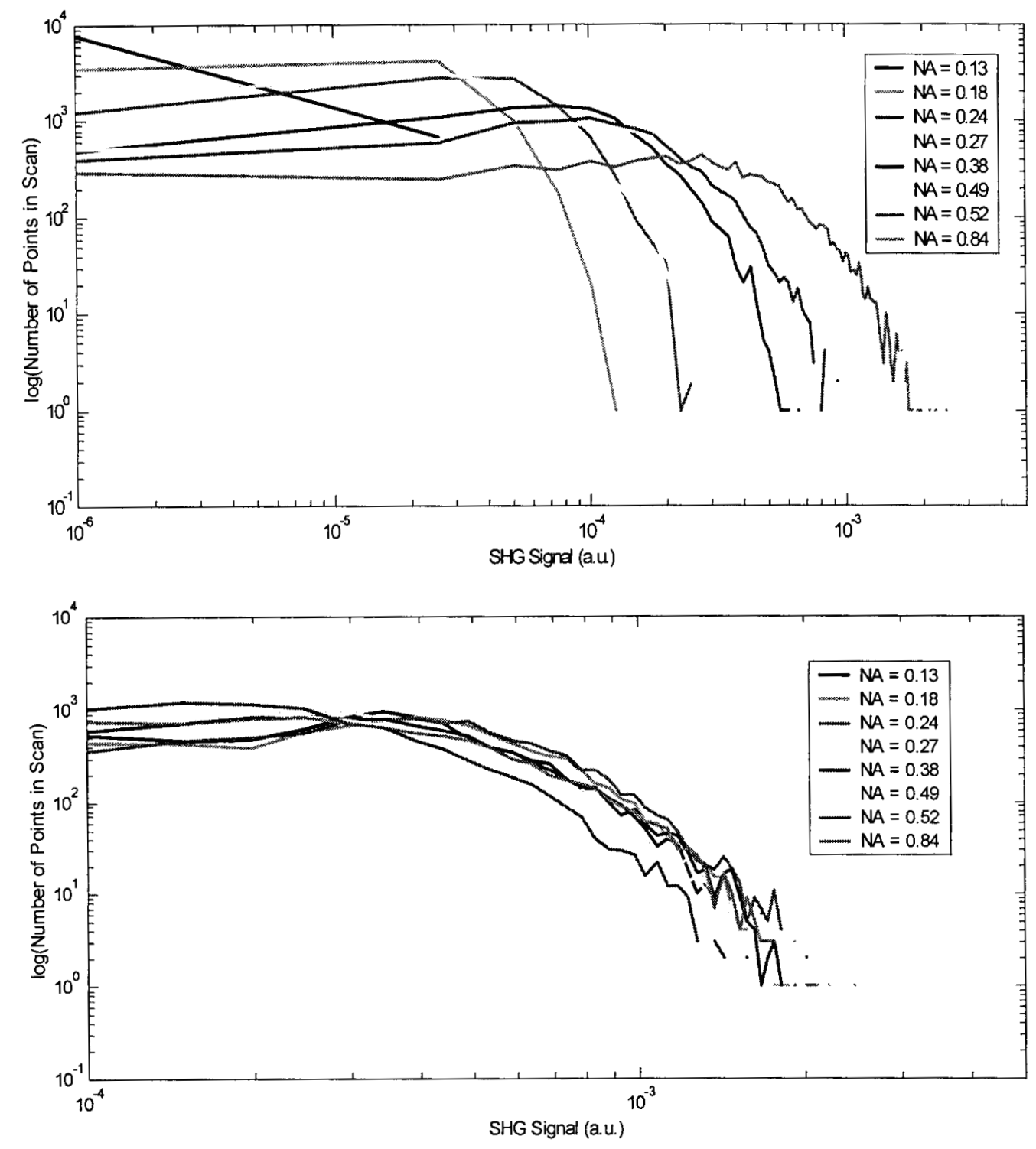

Figure 6.7. Log-log plot of the frequency of occurrence versus second harmonic signal intensity for the images shown in Figure 6.5. The data is plotted using different colors for each of the objective / collimating lens combinations. The NA calculated from the measurements in the quartz waveplate is given for each objective (refer to Table 5.4). In (a) the intensity data is not scaled; in (b) the intensity data was scaled to compensate for the fact that intensity increases in inverse proportion to the square of the focal spot size. The intensity data was divided into 200 equally sized bins spanning the range of $10^{-6}$ to 5 $\times 10^{-3} \mathrm{mV}$. 


\section{Lower bound on the nonlinear susceptibility}

As discussed in Chapter 5, the SHG signal strength in the quartz waveplate can be used to calibrate the experimental setup; this allows measurement of the second order nonlinear susceptibility in a given sample. In Chapter 5, Eq. 5.3 was derived to relate the second order nonlinear susceptibility in collagen to that in quartz, and it is repeated here:

$$
d_{\text {eff(collagen) }}^{2}=\frac{V_{\text {collogen }}\left|J_{\text {quartz }}\left(z_{k}, z_{f \max }, \Delta k\right)\right|^{2}}{V_{\text {quartz }}\left|J_{\text {collagen }}\left(z_{R}, z_{f \max }, \Delta k\right)\right|^{2}} d_{\text {eff( (quartz) }}^{2}
$$

In rat-tail tendon collagen, the degree of parallel and anti-parallel orientation of neighboring fibrils can strongly influence the strength of the detected signal (see above). Therefore, it is only possible to calculate a lower bound on the second order non-linear susceptibility. To obtain this lower bound, the SHG signal must first be measured in both quartz and collagen. In quartz, the peak signal obtained when the laser beam is focused on the front surface of the waveplate is used. In collagen, the peak signal obtained in the rat-tail tendon scans described above is used.

Using Eqs. 3.7, 3.9, 3.12-3.13, and 5.3, and taking into account the fact that rattail tendon is only $60 \%$ collagen (Parry and Craig, 1977), $d_{X X X}$ and $d_{X Y Y}=d_{Y X Y}=d_{Y Y X}$, can be calculated from these measurements. Note that $\mathrm{X}$ represents the direction of the fibril axis and $\mathrm{Y}$ the direction normal to the fibril and beam propagation direction. The signal at the chopper frequency as well as the signal at the first modulation harmonic of the electro-optic modulator can be used to perform this calculation-the signal at the second modulation harmonic of the electro-optic modulator was not used because it is 
significantly weaker. Values for $d_{X X X}$ between $0.13 \mathrm{pm} / \mathrm{V}$ and $0.26 \mathrm{pm} / \mathrm{V}$ were obtained. Values for $d_{X Y Y}=d_{Y X Y}=d_{Y Y X}$ were obtained using Eq. 3.6:

$$
d_{X Y Y}=\gamma d_{X X X} / 3 \gamma+1 .
$$

The results are summarized in Table 6.1 (using data obtained at the chopper frequency) and Table 6.2 (using data obtained at the electro-optic modulator frequency). The highest value of $d_{X X X}$ was obtained for the highest numerical aperture we used $(\mathrm{NA}=0.9)$, while the value obtained for $d_{X X X}$ was roughly halved in magnitude and the same for all of the other numerical apertures. This suggests that at lower numerical aperture (or for a focal spot size of greater than $1.5 \mu \mathrm{m}$ ) significant averaging is occurring over regions with strong SHG signal and regions with weaker SHG signal. For a focal spot size of less than $1.5 \mu \mathrm{m}$, collagen fibrils in the focus may have predominantly one polarity or anotherleading to coherent SHG and thus an enhanced signal. It should, however, be noted that the peak signal in a single $100 \mu \mathrm{m}$ by $100 \mu \mathrm{m}$ image of part of a tendon section typically occurs at only a few isolated points.

Table 6.1. A lower bound on the d-tensor in collagen (signal at chopper frequency)

\begin{tabular}{|c|c|c|c|c|c|}
\hline $\begin{array}{l}\text { Microscope } \\
\text { objective }\end{array}$ & NA & $\begin{array}{l}\text { Peak signal in } \\
\text { collagen } \\
\text { (chopper) }\end{array}$ & $\begin{array}{l}\text { Peak signal in } \\
\text { quartz }\end{array}$ & $\begin{array}{l}d_{x x x} \\
(\mathrm{pm} / \mathrm{V})\end{array}$ & $\begin{array}{l}\mathbf{d}_{\mathrm{XYY}}, \mathbf{d}_{\mathrm{YXY}}, \\
\mathrm{d}_{\mathrm{YYX}} \\
(\mathrm{pm} / \mathrm{V}) \\
\end{array}$ \\
\hline $10 \mathrm{X}(100 \mathrm{~mm})$ & 0.13 & $0.05 \mathrm{mV}$ & $0.19 \mathrm{mV}$ & 0.13 & 0.09 \\
\hline $10 \mathrm{X}(200 \mathrm{~mm})$ & 0.24 & $0.26 \mathrm{mV}$ & $0.54 \mathrm{mV}$ & 0.15 & 0.10 \\
\hline $20 \mathrm{X}(50 \mathrm{~mm})$ & 0.18 & $0.13 \mathrm{mV}$ & $0.32 \mathrm{mV}$ & 0.15 & 0.10 \\
\hline $20 \mathrm{X}(100 \mathrm{~mm})$ & 0.27 & $0.28 \mathrm{mV}$ & $0.54 \mathrm{mV}$ & 0.15 & 0.10 \\
\hline $20 \mathrm{X}(200 \mathrm{~mm})$ & 0.49 & $1.00 \mathrm{mV}$ & $1.07 \mathrm{mV}$ & 0.16 & 0.11 \\
\hline $40 \mathrm{X}(50 \mathrm{~mm})$ & 0.38 & $0.66 \mathrm{mV}$ & $0.89 \mathrm{mV}$ & 0.16 & 0.11 \\
\hline
\end{tabular}




\begin{tabular}{llllll}
\hline $40 \mathrm{X}(100 \mathrm{~mm})$ & 0.52 & $0.94 \mathrm{mV}$ & $1.26 \mathrm{mV}$ & 0.14 & 0.10 \\
\hline $40 \mathrm{X}(200 \mathrm{~mm})$ & 0.85 & $2.50 \mathrm{mV}$ & $0.74 \mathrm{mV}$ & 0.25 & 0.17 \\
\hline
\end{tabular}

Table 6.2. A lower bound on the d-tensor in collagen (signal at EOM frequency)

\begin{tabular}{llllll}
\hline $\begin{array}{l}\text { Microscope } \\
\text { objective }\end{array}$ & NA & $\begin{array}{l}\text { Peak signal in } \\
\text { collagen } \\
\text { (FMH) }\end{array}$ & $\begin{array}{l}\text { Peak signal in } \\
\text { quartz }\end{array}$ & $\begin{array}{l}\mathbf{d}_{\mathbf{X X X}} \\
\mathbf{p m} / \mathbf{V})\end{array}$ & $\begin{array}{l}\mathbf{d}_{\mathbf{X Y Y}}, \mathbf{d}_{\mathbf{Y X Y}} \\
\mathbf{d}_{\mathbf{Y Y X}} \\
(\mathbf{p m} / \mathbf{V})\end{array}$ \\
\hline $10 \mathrm{X}(100 \mathrm{~mm})$ & 0.13 & $0.007 \mathrm{mV}$ & $0.18 \mathrm{mV}$ & 0.13 & 0.09 \\
\hline $10 \mathrm{X}(200 \mathrm{~mm})$ & 0.24 & $0.039 \mathrm{mV}$ & $0.52 \mathrm{mV}$ & 0.15 & 0.10 \\
\hline $20 \mathrm{X}(50 \mathrm{~mm})$ & 0.18 & $0.019 \mathrm{mV}$ & $0.32 \mathrm{mV}$ & 0.14 & 0.10 \\
\hline $20 \mathrm{X}(100 \mathrm{~mm})$ & 0.27 & $0.044 \mathrm{mV}$ & $0.52 \mathrm{mV}$ & 0.15 & 0.10 \\
\hline $20 \mathrm{X}(200 \mathrm{~mm})$ & 0.49 & $0.157 \mathrm{mV}$ & $1.06 \mathrm{mV}$ & 0.16 & 0.11 \\
\hline $40 \mathrm{X}(50 \mathrm{~mm})$ & 0.38 & $0.100 \mathrm{mV}$ & $0.82 \mathrm{mV}$ & 0.16 & 0.11 \\
\hline $40 \mathrm{X}(100 \mathrm{~mm})$ & 0.52 & $0.148 \mathrm{mV}$ & $1.23 \mathrm{mV}$ & 0.14 & 0.10 \\
\hline $40 \mathrm{X}(200 \mathrm{~mm})$ & 0.85 & $0.417 \mathrm{mV}$ & $0.73 \mathrm{mV}$ & 0.26 & 0.17 \\
\hline
\end{tabular}

\section{Discussion}

The large variation in signal strength within highly organized, uniform tissue suggests that it would be difficult to use the strength of the second harmonic signal alone to detect subtle changes in collagen structure. This work has focused on rat-tail tendon collagen. Could collagen fibrils in other tissues be organized parallel to each other? Extensive studies using electron microscopy, in tissues that include tendon, dermis, and cornea, have found no instance where this is the case (Scott and Parry, 1992; Birk et. al., 1996). In fact, the random orientation may be an important part of a possible mechanism for the control of fibril diameter: neighboring parallel, but not anti-parallel, fibrils are allowed to fuse (Scott, 1990).

In the next two chapters, the polarization dependence of the second harmonic signal - which is independent of the parallel or anti-parallel orientation of neighboring fibrils-is investigated to determine if it can provide information about changes in collagen structure that the magnitude of the signal cannot. 


\section{References}

E. Baer, J. J. Cassidy, and A. Hiltner. 1988. Hierarchical structure of collagen and its relationship to the physical properties of tendon. In Collagen: Biochemistry and Biomechanics. Volume 2. Marcel E. Nimni, editor. CRC Press, Boca Raton. 177199.

D. E. Birk, R. A. Hahn, C. Y. Linsenmayer, and E. I. Zycband. 1996. Characterization of collagen fibril segments from chicken embryo cornea, dermis, and tendon. Matrix Biology. 15:111-118.

I. Freund, M. Deutsch, and A. Sprecher. 1986. Connective tissue polarity: Optical second-harmonic microscopy, crossed-beam summation, and small-angle scattering in rat-tail tendon. Biophysical Journal. 50:693-712.

D. A. D. Parry, and A. S. Craig. 1977. Quantitative electron microscope observations of the collagen fibrils in rat-tail tendon. Biopolymers. 16:1015-1031.

J. E. Scott. 1990. Proteoglycan: collagen interactions and subfibrillar structure in collagen fibrils. Implications in the development and ageing of connective tissues. Journal of Anatomy. 169:23-35.

J. E. Scott and D. A. D. Parry. 1992. Control of collagen fibril diameters in tissues. International Journal of Biological Macromolecules. 14:292-293. 


\section{SHG POLARIZATION DEPENDENCE: THE EFFECT OF CHANGES IN COLLAGEN STRUCTURE AND CHEMISTRY}

\section{Background and overview of the experiment}

In Chapter 6, the magnitude of the second harmonic signal was measured in thin sections of rat-tail tendon. Large variations in signal strength were observed even in these highly uniform tissue samples, making it difficult to detect any changes in collagen chemistry or structure that introduce only a relatively small change in signal strength. The magnitude of the second harmonic signal is, however, not the only information about the second order nonlinear susceptibility that is obtained from SHG microscopy. Polarization-modulated SHG microscopy allows two dependent parameters, $R$ and $S$ (see Chapter 3), both functions of the ratio of the two independent elements of the second order nonlinear susceptibility tensor, to be measured. If biologically relevant changes in collagen structure lead to changes in $R$ and $S$, this information could potentially be used to detect pathological conditions that involve collagen. This chapter investigates the effects of perturbations of collagen structure, induced under controlled, in vitro conditions, on $R$ and $S$. The effect of thermal denaturation, enzymatic digestion, nonenzymatic crosslinking, and hydration are discussed.

Isolated rat-tail tendon fascicles were subjected to conditions designed to produce chemical and structural changes analogous to those that occur in vivo. To minimize effects related to changes in the linear optical properties of collagen such as birefringence and polarization-dependent scattering, thin sections of tissue were used (refer to Chapter 2). Additional experiments designed to determine if fibrils formed from genetically distinct collagen types had different values of $R$ and $S$ were also conducted. 
Measurements of $R$ were made using the 20X objective (Table 4.1 ) and $200 \mathrm{~mm}$ focal length collimating lens. Measurements of $S$ were made to confirm that the measured values of $R$ and $S$ (both a function of $\gamma$ ) were consistent with each otherlending additional support to the cylindrical symmetry model of SHG in collagen fibrils. Measurements using different microscope objectives to focus the beam were also made to determine if numerical aperture affected the measured values of $R$. Finally, in order to demonstrate that these measurements can also be made successfully in reflection mode, measurements made in both transmission mode and reflection mode were compared. 


\section{Sample preparation}

\section{Tissue source}

Type I collagen fascicles were obtained as discussed in Chapter 6. Some fascicles were structurally modified, as described below. Both control and modified samples were prepared for analysis as frozen sections.

\section{Nonenzymatic crosslinking}

Several agents were used for inducing covalent crosslinking in collagen: glutaraldehyde, formalin, methylglyoxal (MG), and glucose-6-phosphate. Glutaraldehyde and formaldehyde are potent chemical crosslinking agents used for tissue fixation; they were selected because they are capable of producing far higher levels of covalent bonds than occur naturally. Glucose-6-phosphate reacts with lysine residues on collagen nonenzymatically; over time these adducts undergo a series of complex reactions and rearrangements, culminating in the formation of advanced glycation-endproducts (AGEs). AGEs comprise a heterogeneous group of compounds, covalently bonded to collagen, that accumulate on collagen in virtually all tissues and organ systems, and which are associated with the profound structural and functional changes that occur in aging and diabetes (Reiser, 1998). G-6-P was selected as a glycating agent because it reacts with collagen in vitro much more quickly than glucose. MG is a naturally occurring metabolic intermediate, which has recently been recognized as playing a role in AGE formation in vivo. In vitro studies have shown that incubation of collagen with MG results in rapid formation of AGE compounds. We selected this compound in order to generate collagen with a high level of AGE-type crosslinking. 
Samples were placed in microcentrifuge vials to which were added one $\mathrm{ml}$ of one of the following solutions: $10 \%$ formalin, $5 \%$ glutaraldehyde in $500 \mathrm{mM}$ sodium phosphate buffer, $\mathrm{pH} 7.1 ; 100 \mathrm{mM}$ methylglyoxal in $250 \mathrm{mM}$ sodium phosphate buffer, pH 7.1.; $200 \mathrm{mM}$ glucose-6-phosphate in $250 \mathrm{mM}$ sodium phosphate buffer, $\mathrm{pH}$ 7.1. Control samples were incubated in buffer alone. All of the samples except for those incubated with G-6-P were incubated for 24 hrs at room temperature. Samples in G-6-P were incubated for 1,2 , or 3 weeks. At the end of the incubation period, all samples were washed in phosphate buffered saline (PBS) and sectioned as described above.

\section{Age dependent crosslinking}

To examine the effects of age on SHG, a tail tendon sample from a 30 month old rat (available from a previous study) that had been stored at -20 degrees $\mathrm{C}$ was obtained. Frozen sections were prepared, and serial sections were obtained from $20 \mu \mathrm{m}$ to $30 \mu \mathrm{m}$ below the surface and mounted, unstained, on glass slides beneath a cover slip.

\section{Thermal denaturation}

Rat tail tendon fascicles were placed in microcentrifuge vials to which was added $1 \mathrm{ml}$ of PBS at room temperature. The vials were placed for 45 minutes in a digital dry bath heater (Isotemp 125D; Fisher Scientific, Houston, TX), that had already reached its set temperature. The temperature display on the digital dry bath heater was calibrated against an $\mathrm{Hg}$ thermometer. The following temperatures were used: 50.0 degrees $\mathrm{C}, 51.0$

degrees C, 52.0 degrees C, 52.5 degrees C, 53.0 degrees C, 53.5 degrees C, 54.0 degrees C, 55.0 degrees C, 56.0 degrees C, 56.5 degrees C, and 57.0 degrees C. A control sample 
was heated to 40 degrees $\mathrm{C}$ for 45 minutes. At the end of the heating period, the vials were removed from the heating block and allowed to cool to room temperature.. Frozen sections were prepared as described above. In a second experiment, vials with rat-tail tendon fascicles were placed in the digital dry bath heater for different lengths of time (2 $\min , 3 \mathrm{~min}, 3.5 \mathrm{~min}, 4 \mathrm{~min}, 5 \mathrm{~min}$, and $10 \mathrm{~min}$ ) but at a fixed dry bath temperature of 65 degrees $\mathrm{C}$.

\section{Alcohol drying}

To determine the effect of collagen hydration on SHG, normal fascicles were compared with fascicles that had been subjected to dehydration through immersion in a graded series of ethanol dilutions, beginning with $25 \%$ ethanol in water, and ending in absolute ethanol.

\section{Collagenase digestion}

A solution of highly purified collagenase (Type VII collagenase; Sigma, Saint Louis, MO), containing $140 \mathrm{U} / \mathrm{ml}(1000-3000 \mathrm{CDU} / \mathrm{mg})$ in $0.01 \mathrm{M} \mathrm{CaCl}_{2} / 0.02 \mathrm{M}$ Tris $(\mathrm{pH} 7.55) / 0.05 \%$ toluene was prepared. Rat-tail tendon fascicles were incubated at 37 degrees $\mathrm{C}$ in this solution for $0 \mathrm{~h}, 2 \mathrm{~h}, 4 \mathrm{~h}, 6 \mathrm{~h}$, and $12 \mathrm{~h}$.

\section{Fibrillogenesis from soluble collagen}

Collagen fibrils were also generated in vitro from different types of collagen (types I, III, and V). Purified, soluble Type I collagen was purchased commercially (Sigma, Saint Louis, MO). Purified type III and type V collagen were obtained from Dr. 
Karen Reiser. Collagen of each type was dissolved in $20 \mathrm{mM} \mathrm{NaPO}_{4}$. The solution was incubated for about 1 day at 38 degrees $\mathrm{C}$ using a stirring hot plate to allow fibrillogenesis to occur. In some cases, the solution was incubated for up to one week in order to allow crosslinking to occur, as has been previously described (Gelman et. al., 1979). The presence of crosslinks (formed from aldehydes already present on the soluble collagen) was confirmed by observing the effects of reversing the conditions. Individual fibers-bundles of collagen fibrils approximately $10 \mu \mathrm{m}$ in diameter-were separated from solution and mounted on a slide beneath a cover slip. 


\section{Results}

Collagen is characterized not only by its well-defined structure-from the molecular to the macroscopic level—but also by its continuous modification years after the initial structure has been assembled. We wanted to investigate the effect of some of these modifications on SHG signal under controlled conditions. To this end, isolated fascicles were subjected to conditions designed to produce perturbations in structural integrity analogous to those that occur in vivo. The effects of increased nonenzymatic glycation, enzymatic digestion, and thermal denaturation were examined. In addition, the effects of inducing high levels of covalent crosslinking with agents such as glutaraldehyde and formaldehyde were studied. The effect of these changes on the polarization dependence of the SHG signal was determined by measuring $R$. Figure 7.1 shows a curve of the SHG signal as a function of polarization angle (obtained by applying different DC voltages across the EOM and measuring the SHG signal at a single point in a section of rat-tail tendon) together with a curve calculated from a corresponding measurement of $R$ (obtained using the polarization-modulation technique). Figure 7.1 illustrates that all of the information about the polarization dependence of the second harmonic signal is obtained by measuring $R$. 


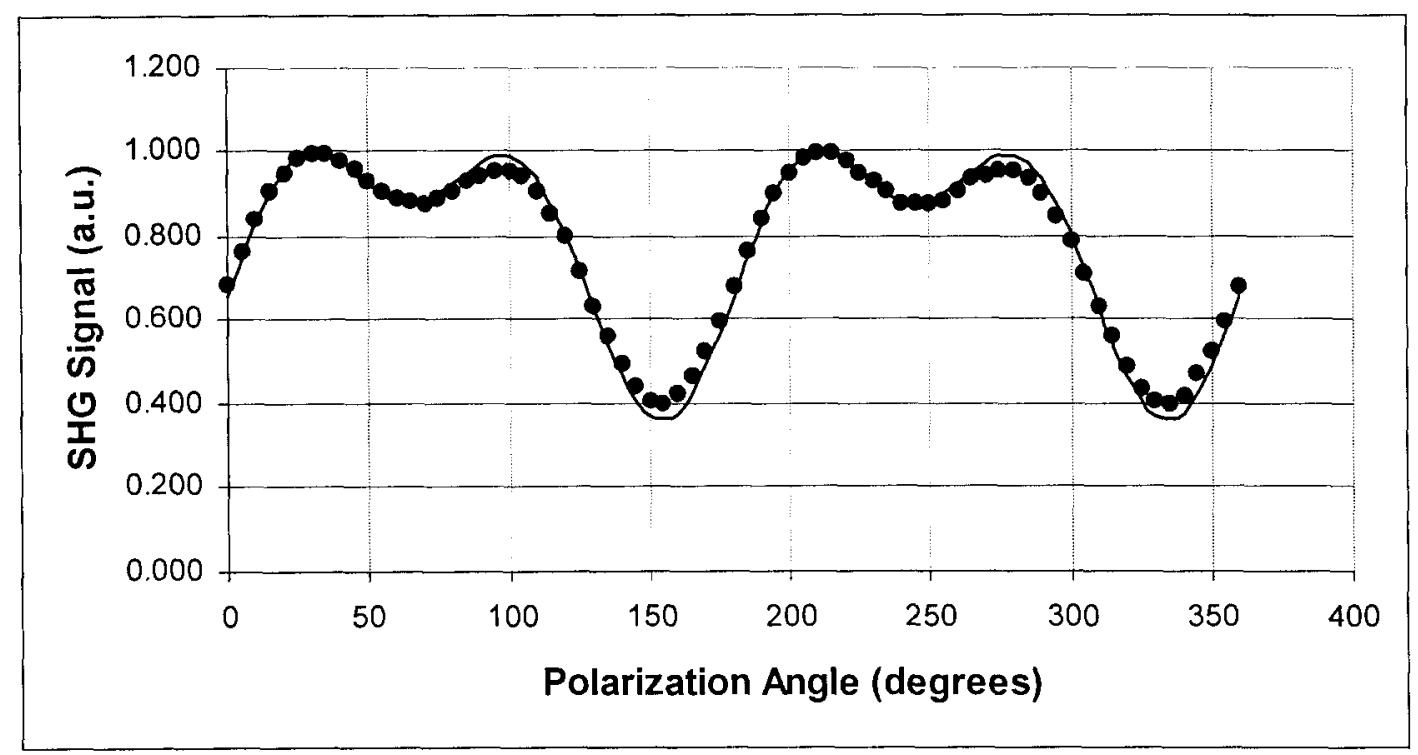

Figure 7.I. Plot of the polarization dependence of the second harmonic signal obtained (circles) by adjusting the polarization by changing a DC voltage across the EOM and by using the polarization-modulation technique to measure $R$ (curve).

\section{Nonenzymatic crosslinking}

$R$ was measured in sections from rat tail tendon tissue crosslinked with methylglyoxal (MG), glucose-6-phosphate, glutaraldehyde or formaldehyde. For each of the samples, three regions to scan were chosen at random. However, regions where the section was folded over on itself, crumpled, or torn were avoided since such phenomena can have a significant effect on $R$ (Figure 7.2). Each region consisted of a $150 \mu \mathrm{m}$ by $100 \mu \mathrm{m}$ rectangle in the plane of the frozen section. Data were collected using the $20 \mathrm{X}$ objective and with a scan resolution of $2 \mu \mathrm{m}$ (15,000 points per region). Data points where the signal at the first modulation harmonic did not exceed a minimum threshold (about ten times the noise floor) were eliminated. These results are summarized in Figure 7.3. There were no significant differences between control collagen and any of the crosslinked collagen with respect to $R$. $R$ was also measured in collagen obtained from 
an old rat, to determine if AGE accumulation that had occurred naturally over an extended period of time might differ in some way from crosslinking induced artificially at an accelerated rate. However, $R$ in aged tissue was not significantly different from that of control tissue (Figure 7.3).
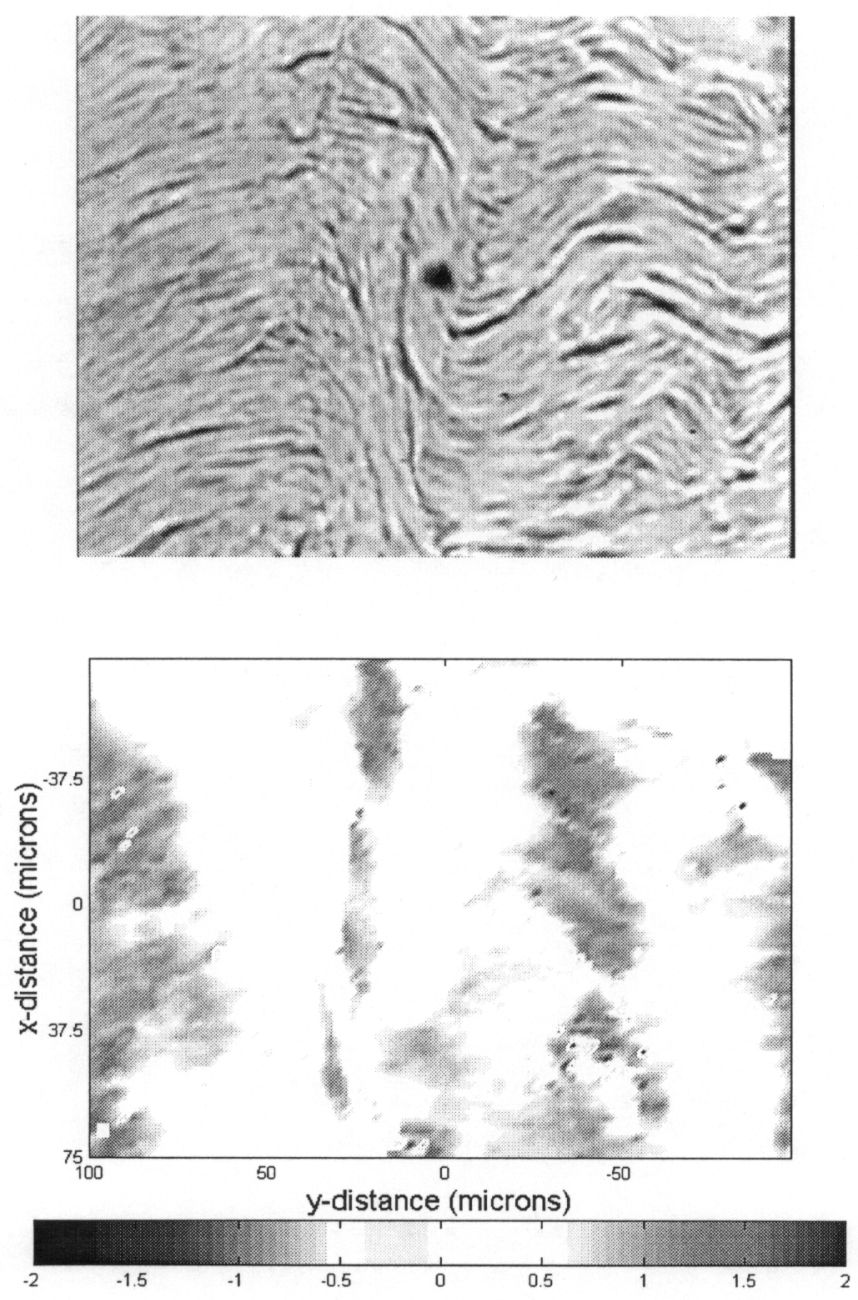

Figure 7.2. (a.) Visible light image of a small region of a frozen section of rat-tail tendon fascicle. The image shows how parts of the section have been folded and twisted. (b.) Measurement of $R$ in the same region as (b), plotted as a function of position in the sample. 


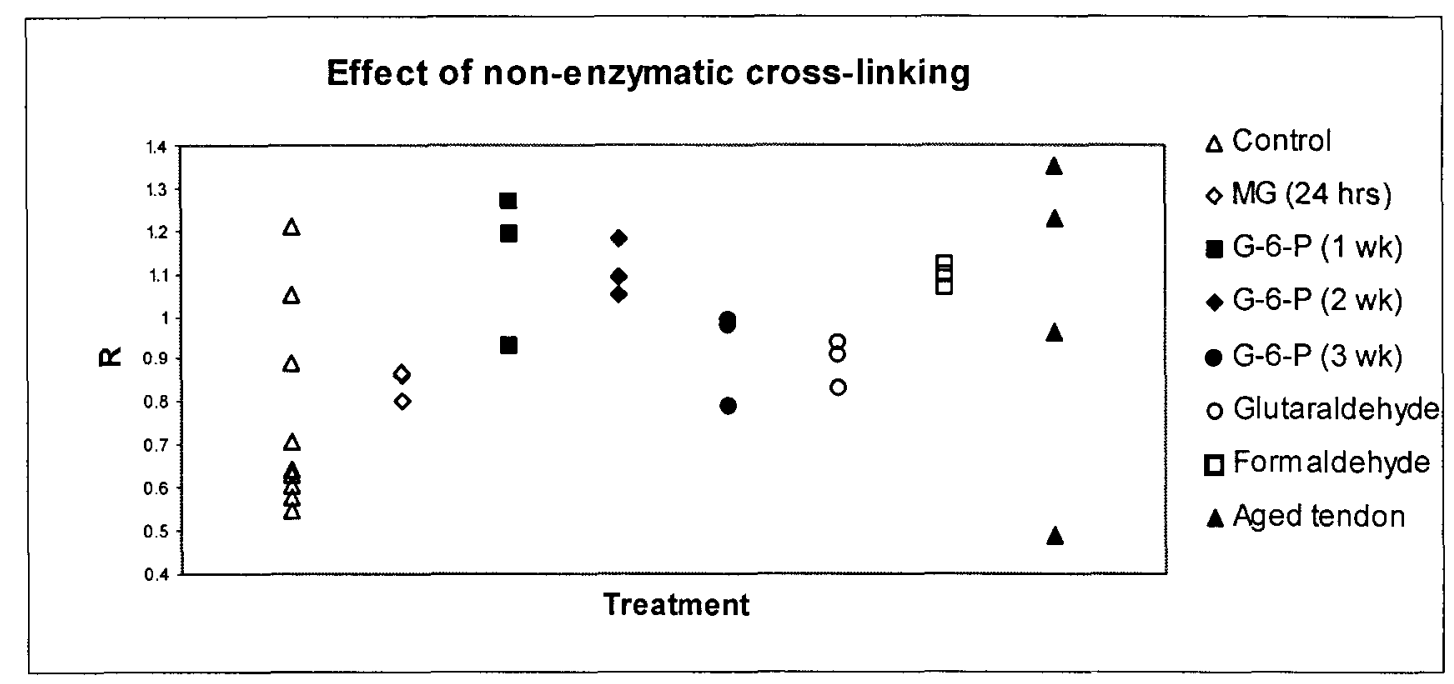

Figure 7.3. Scatter-plot of the effect on $R$ of nonenzymatic crosslinking. The mean value measured for each region scanned is plotted as a single point.

\section{Thermal denaturation}

Samples subjected to temperatures less than $\sim 52$ degrees $C$ for 45 minutes had a normal SHG signal; samples heated to a temperature exceeding $\sim 54$ degrees $\mathrm{C}$ had no SHG signal. Some samples heated to $\sim 53.0$ degrees $\mathrm{C}$ had no signal, while others had normal signal. Samples heated for 45 minutes had either a normal SHG signal or no signal at all, suggesting a sharp transition around 53 degrees. Similarly, samples heated to 65 degrees $\mathrm{C}$ for less than 3 minutes had normal signal, while samples heated to 65 degrees $\mathrm{C}$ for longer times had no signal.

For each sample, $R$ was measured in three randomly selected $100 \mu \mathrm{m}$ by $100 \mu \mathrm{m}$ regions (20X objective, $1 \mu \mathrm{m}$ scan resolution). The effects of heat on $R$ (in those samples in which signal was detectable) are shown in Figure 7.4. There is no significant difference (with respect to $R$ ) between the heated samples and the control samples. 


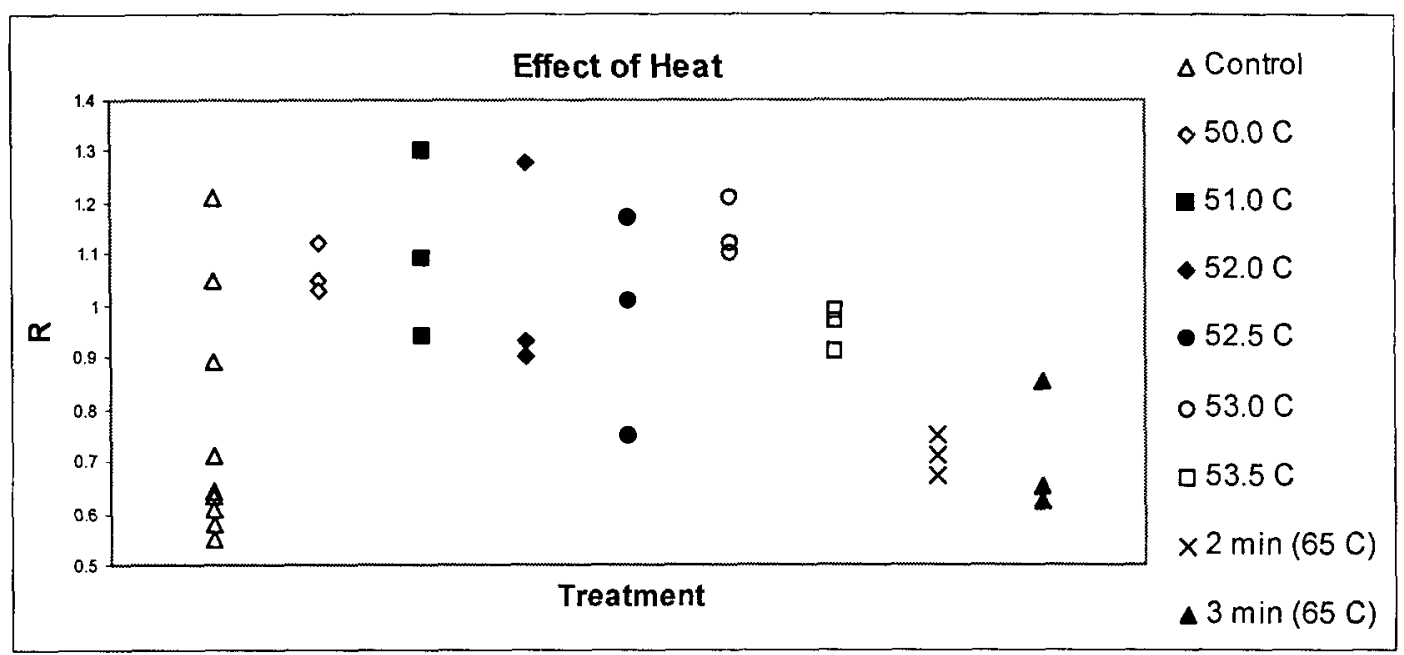

Figure 7.4. Scatter-plot of the effect on $R$ of heating the samples. Unless indicated otherwise, samples were heated for 45 minutes. The mean value measured for each region scanned is plotted as a single point. Note that two different samples were heated to 51.0 degrees $C$ and 53.0 degrees $C$; samples heated to 54.0 degrees $C, 55.0$ degrees $C$, 56.0 degrees $C$, 56.5 degrees $C$, and 57.0 degrees $C$ had no detectable signal. Samples heated to 65.0 degrees for longer than 3.5 minutes, 4 minutes, 5 minutes, and 10 minutes also had no detectable signal.

\section{Collagenase digestion}

SHG signal was measured in collagen digested with collagenase at 37 degrees $\mathrm{C}$ for different time periods. Tissue treated for $4 \mathrm{~h}$ had normal signal; no signal was detectable in any tissue treated for $6 \mathrm{~h}$ or longer. For each sample, $R$ was measured in three randomly selected $50 \mu \mathrm{m}$ by $50 \mu \mathrm{m}$ regions (20X objective, $1 \mu \mathrm{m}$ scan resolution). The effects of collagenase digestion on $R$ are shown in Figure 7.5. There is no significant difference with respect to $R$ between the collagenase digested samples and the control samples. 


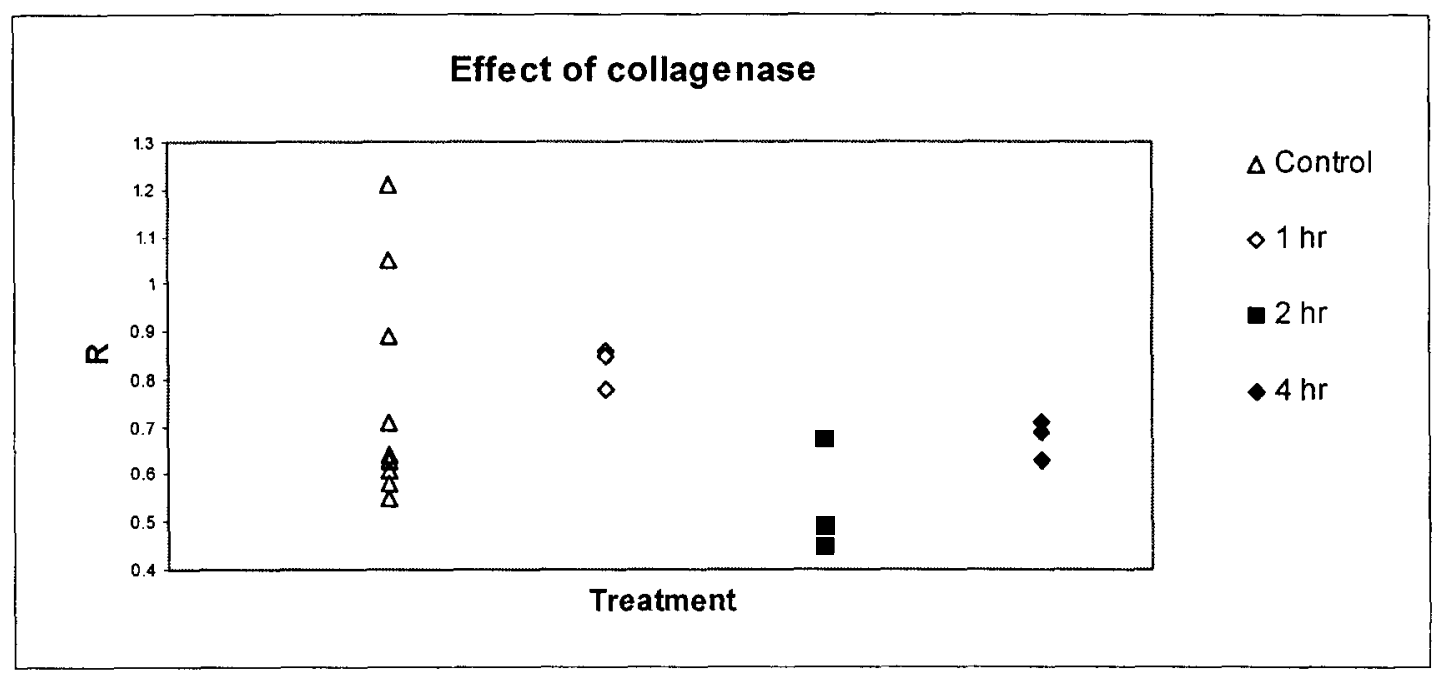

Figure 7.5. Scatter-plot of the effect on $R$ of collagenase. The mean value measured for each region scanned is plotted as a single point. Samples incubated with collagenase for $6 \mathrm{hr}, 12 \mathrm{hr}$, and $24 \mathrm{hr}$ had no detectable signal.

\section{Hydration state}

To determine the effect of hydration state, the second harmonic signal in sections embedded in paraffin (a technique requiring dehydration through graded ethanol baths) was compared with that observed in frozen sections (a technique which involves no dehydration steps). $R$ was measured in six randomly selected $100 \mu \mathrm{m}$ by $100 \mu \mathrm{m}$ regions ( $1 \mu \mathrm{m}$ scan resolution, 20X objective) of the paraffin sections. The value of $R$ in the paraffin sections was lower than that in the frozen sections (Figure 7.6). 


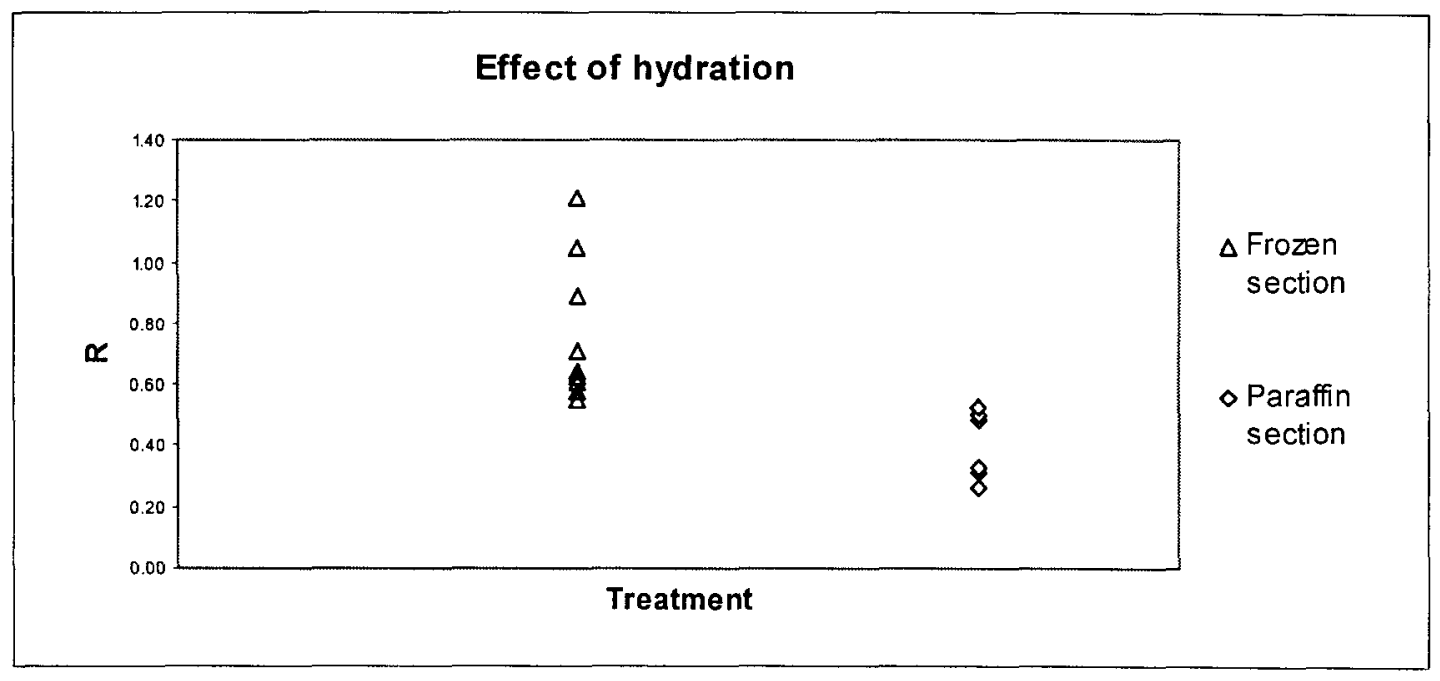

Figure 7.6. Scatter-plot of the effect on $R$ of hydration. The mean value measured for each region scanned is plotted as a single point.

\section{Genetically distinct collagen fibrils}

$R$ was also measured in collagen fibrils prepared from soluble type I, type III, and type $\mathrm{V}$ collagen to determine if the type of collagen influenced the polarization dependence of the second harmonic light generated. The fibril bundles that formed after fibrillogenesis (incubation at 38 degrees $\mathrm{C}$ for one day) differed somewhat in diameter ( $10 \mu \mathrm{m}$ for type III and $\sim 20 \mu \mathrm{m}$ for type I and type V), but all were well-organized with fibrils aligned parallel to the bundle axis. Allowing fibrillogenesis to occur over a longer time period by extending the incubation time by several days led to the formation of fibrils with larger diameters.

$R$ in the type I collagen fibrils assembled in vitro was substantially lower than $R$ in the native type I collagen fibrils of rat-tail tendon (an average value of $R=0.27 \pm 0.14$ was measured). This suggests a significant structural difference between collagen formed in vivo and collagen formed in vitro. Initially, it was suspected that the absence of enzymatic crosslinking in the collagen fibrils formed in vitro might be responsible for 
this difference. However, it was found that allowing enzymatic crosslinks to re-form did not lead to a significant increase in $R$. In type III collagen fibrils formed in vitro, $R=$ $0.18 \pm 0.11$ was measured and in type $\mathrm{V}$ collagen fibrils formed in vitro, $R=0.15 \pm 0.08$ was measured.

\section{Effect of numerical aperture on measurement of $R$}

In all of the experiments discussed above, the $20 \mathrm{X}$ objective and the $200 \mathrm{~mm}$ collimating lens were used. Could the numerical aperture significantly affect the measured value of $R$ ? The average values and standard deviations of $R$ were calculated from measurements of the second harmonic signal at the first and second modulation harmonics in a $100 \mu \mathrm{m}$ by $100 \mu \mathrm{m}$ region of a frozen section of rat-tail tendon (the same tissue section used to produce the images in Figure 6.5). The results are summarized in Table 7.1. The averages of the measured values obtained with each microscope objective and collimating lens combination range between $R=1.22$ and $R=1.44$, a spread of only about $15 \%$. Changing the beam focus, even using a numerical aperture of 0.9 (in the case of the $40 \mathrm{X}$ objective used with the $200 \mathrm{~mm}$ collimating lens) did not have a significant impact on the measured value of $R$.

Table 7.1. SHG in rat-tail tendon frozen sections for different microscope objectives and collimating lenses

\begin{tabular}{lll}
\hline Microscope objective & Collimating lens & R: mean \pm std. dev. \\
\hline $10 \mathrm{X}$ & $\mathrm{f}=100 \mathrm{~mm}$ & $1.30 \pm 0.29$ \\
\hline $10 \mathrm{X}$ & $\mathrm{f}=200 \mathrm{~mm}$ & $1.28 \pm 0.16$ \\
\hline $20 \mathrm{X}$ & $\mathrm{f}=50 \mathrm{~mm}$ & $1.22 \pm 0.16$ \\
\hline $20 \mathrm{X}$ & $\mathrm{f}=100 \mathrm{~mm}$ & $1.24 \pm 0.16$ \\
\hline $20 \mathrm{X}$ & $\mathrm{f}=200 \mathrm{~mm}$ & $1.30 \pm 0.17$ \\
\hline $40 \mathrm{X}$ & $\mathrm{f}=50 \mathrm{~mm}$ & $1.38 \pm 0.22$ \\
\hline $40 \mathrm{X}$ & $\mathrm{f}=100 \mathrm{~mm}$ & $1.33 \pm 0.21$ \\
\hline $40 \mathrm{X}$ & $\mathrm{f}=200 \mathrm{~mm}$ & $1.44 \pm 0.31$ \\
\hline
\end{tabular}




\section{Effect of using reflection mode instead of transmission mode microscopy on $R$}

Measurements of $R$ were made in the same rat-tail tendon frozen section after modifying the system to allow reflection-mode detection of the second harmonic signal (as described in Chapter 4). In the case of thin sections, most of the second harmonic signal measured in reflection mode is likely probably generated in the forward direction and reflected at the interface between the sample and the slide (or the slide and the air). Since this reflection occurs at near-normal incidence, it should be independent of polarization and measurements of $R$ should be the same in both transmission- and reflection-mode.

The $20 \mathrm{X}$ objective and $200 \mathrm{~mm}$ collimating lens were used in this experiment. In transmission mode, the addition of the mirror led to a small change: $R=1.37 \pm 0.27$ (before, $R=1.30 \pm 0.17$ was measured). In reflection mode $\mathrm{R}=1.27 \pm 0.55$ was measured. The significantly larger spread in $R$ measured in reflection mode can probably be attributed to the fact that the reflected signal is significantly weaker.

\section{$R, S$, and $\gamma$}

The results presented above focused on measuring $R$ - the ratio between the second harmonic signal modulated at double the electro-optic modulator frequency and the signal modulated at the electro-optic modulator frequency. From a measured value of $R$, it is possible to directly calculate $\gamma$, a parameter of the second order nonlinear susceptibility tensor of collagen. However, as discussed in Chapter 2, it is also possible to measure the ratio $S$ between the signal at the electro-optic modulator frequency and the signal at the chopper frequency. It was noted previously (see Chapter 3) that the 
measured value of $S$ must be corrected to in order to take into account the fact that the chopper modulation is not a sine wave; measurements in quartz were used to obtain this correction factor. Once $S$ has been corrected, it can also be used to calculate $\gamma$. Confirmation for the cylindrical symmetry model of collagen is obtained if the values of $\gamma$ calculated from measurements of $R$ and $S$ agree. To obtain this confirmation, both $R$ and $S$ were measured in the same region of the sample shown in Figure 6.5; mean values of $R=1.30$ and $S=0.15$ were obtained (using the $20 \mathrm{X}$ objective and $200 \mathrm{~mm}$ collimating lens). The measured value of $S$ was multiplied by the correction factor (1.42) to obtain $S_{\text {corr }}=0.21$. The parameter $\gamma$ can then be calculated from $R$ to obtain $\gamma=-0.60$; it can also be calculated from $S$-this calculation again gives a value of $\gamma=-0.60$. The good agreement between these two results offers additional support for the cylindrical symmetry model of SHG in collagen.

Finally, given $\gamma$, it is possible using the expression $d_{X Y Y}=\gamma d d_{X X X} / 3 \gamma+1$ (refer to Chapter 2). to calculate the ratio of the two independent elements in the second order nonlinear susceptibility tensor for this example: $d_{X Y Y} / d_{X X X}=0.75$ (X represents the collagen fibril axis and $\mathrm{Y}$ represents the direction normal to the fibril axis and the laser propagation direction; see Chapter 2). This ratio ranges between about 0.60 and 0.75 in all of our control samples; $d_{X Y Y} / d_{X X X}=0.66$ is an average value. 


\section{Discussion}

The effect of several biologically relevant modifications of collagen structurenonenzymatic crosslinking, thermal denaturation, collagenase digestion, and dehydration - on the polarization dependence of SHG has been measured. Nonenzymatic crosslinking had no observable effect on the polarization dependence-whether samples were crosslinked in vitro or whether crosslinking occurred naturally through aging. These findings were surprising, since alterations in crosslinking are known to have significant effects on many aspects of collagen structure. For example, glycation has been shown to significantly increase molecular packing (Tanaka et. al., 1988). Similarly, collagen that has been subjected to strong chemical crosslinking agents such as glutaraldehdye and formaldehyde has extensive alterations in its structure at both the molecular and supramolecular level (Nimni et. al., 1988; Heidemann, 1988). Virtually the only aspect of collagen that remains unchanged by such treatment is its primary structure.

Thermal denaturation resulted in an abrupt drop in the SHG signal, but we did not observe that the polarization dependence of the signal changed in samples heated to just below the point of denaturation. Changes that did not result in destruction of the fibrillar structure did not result in a change in the SHG signal. A previous study of SHG in thermally denatured collagen--using purified type I collagen fibrils, a much larger sample, and a much larger focal spot $(\sim 0.5 \mathrm{~cm})$-found that SHG signal became undetectable at a temperature between 65 degrees $\mathrm{C}$ and 70 degrees $\mathrm{C}$ (Theodossiou et. al., 2002). This study also found that for temperatures of 30 degrees $\mathrm{C}$ to 50 degrees $\mathrm{C}$, the SHG signal dropped at the same rate as the fluorescence signal; at higher temperatures, the SHG signal dropped more rapidly. The larger samples and the use of 
purified collagen may account for the fact that SHG signal was observed at temperatures up to 65 degrees $\mathrm{C}$, a temperature at which samples heated for 45 minutes in this study were completely denatured. The temperature at which Theodossiou et. al. (2002) observed an increase in the rate of SHG decay corresponds to the temperature $(\sim 53$ degrees C) at which tissue was completely denatured in our study.

In the collagenase-digested samples, similar results as in the case of thermal denaturation were obtained. Samples that were digested for $2 \mathrm{hr}$ or $4 \mathrm{hr}$ did not exhibit a SHG polarization dependence that was significantly different from the control samples. A sample that was digested for $6 \mathrm{hr}-$ where the fibrillar structure was destroyed-did not have any SHG signal.

Significant changes in the degree of hydration do have an effect on the polarization dependence of the second harmonic signal. The value of $R$ measured in dry tissue is lower than that in hydrated tissue. What does a low value of $R$ mean physically? Assuming that the effects of birefringence, oblique incidence, and overlapping fibers are small (true for the thin, highly aligned collagen of rat-tail tendon sections), as $R$ approaches $0, \gamma$ approaches negative infinity. Since $\gamma=b / a$ is the ratio of the two independent elements of the second order nonlinear susceptibility tensor, this implies that $a$ goes to zero as $R$ goes to 0 . In the other extreme, for a large ratio, $\gamma$ approaches -0.5 and a approaches $-2 b$. If we take the simple case of a beam polarized at 45 degrees to the fiber axis, $a=0$ implies that (refer back to Eqs 2.9 and 2.10) the ratio between SHG intensity parallel to the fiber and SHG intensity perpendicular to the fiber is $4: 1$. On the other hand, $a=-2 b$ implies that this ratio is $1: 1$. Therefore, as $R$ decreases, the SHG signal produced by an input beam polarized at 45 degrees to the fiber axis becomes 
increasingly polarized along the fiber axis. We suspect, for instance, that in tissue dried in alcohol the reduction in the number of highly polar water molecules reduces the nonlinear polarizability normal to the fiber direction and thus leads to a low value of $R$.

Collagen fibril bundles formed through in vitro fibrillogenesis also had lower values of $R$, even though these structures were not dehydrated in alcohol. Allowing crosslinks to re-form did not lead to a change in $R$. This, together with the fact that nonenzymatic crosslinking - even in extreme cases of treatment with tissue crosslinking agents such as formaldehyde and glutaraldehyde-did not lead to a change in $R$ suggests that the covalent crosslinks may not play a major role in determining the polarization dependence of SHG in collagen. 


\section{Summary}

The effects of structural perturbations on SHG in collagen were assessed in this study. The polarization dependence of the SHG signal was studied because it fluctuates much less than does the magnitude of the signal. Not surprisingly, when collagenase and thermal denaturation destroy the fibrillar structure of collagen, the second harmonic signal is lost completely. The hydration state of collagen significantly affected the polarization dependence of SHG; the absence of highly polarizable water molecules may be responsible for this change. The most surprising finding of this study was the complete absence of any change in SHG polarization dependence in collagen that had been subjected to the most extensive structural modification—short of denaturation—in our study. Unlike hydration, covalent crosslinking of collagen fibrils did not have a detectable effect on the polarization dependence. 


\section{References}

R. A. Gelman, B. R. Williams, and K. A. Piez. 1979. Collagen fibril formation: evidence for a multistep process. Journal of Biological Chemistry. 254, 180-186.

E. Heidemann. 1988. The chemistry of tanning. In Collagen: Biotechnology. Volume 3. Marcel E. Nimni, editor. CRC Press, Boca Raton. 39-61.T. Oya, N. Hattori, Y. Mizuno, S. Miyata, S. Maeda, T. Osawa, and K. Uchida. 1999. Methylglyoxal modification of protein. The Journal of Biological Chemistry. 274:18492-18502.

M. E. Nimni, D. T. Cheung, B. Strates, M. Kodama, and K. Sheikh. 1988. Bioprosthesis derived from crosslinked and chemically modified collagenous tissues. In Collagen: Biotechnology. Volume 3. Marcel E. Nimni, editor. CRC Press, Boca Raton. 1-38.

K. M. Reiser. 1998. Nonenzymatic glycation of collagen in aging and diabetes. Proceedings of the Society of Experimental Biology and Medicine. 218:23-37.

S. Tanaka, G. Avigad, B. Brodsky, and E. F. Eikenberry. 1988. "Glycation induces expansion of the molecular packing of collagen," Journal of Molecular Biology $203: 495-505$.

T. Theodossiou, G. S. Rapti, V. Hovhannisyan, E. Georgiou, K. Politopoulos, and D. Yova. 2002. "Thermally induced irreversible conformational changes probed by optical second harmonic generation and laser-induced fluorescence," Lasers in the Medical Sciences. 17:34-41. 


\section{MEASURING COLLAGEN ORIENTATION USING POLARIZATION-MODULATED SHG}

The previous two chapters presented detailed measurements of the second harmonic signal magnitude and polarization dependence in collagen. These measurements contribute significantly to our understanding of SHG in collagen. They confirm, for instance, that neighboring collagen fibrils are generally oriented parallel and anti-parallel to each other in a random manner, explaining the large variability in signal strength with position. These measurements also suggest that glycation and nonenzymatic crosslinking have little influence on the second order nonlinear susceptibility tensor, while the hydration state of collagen appear to have an important effect. Intact collagen fibrils are necessary for SHG to occur.

An additional aspect of polarization-modulated SHG-the phase of the detected signal with respect to the modulation-provides further information: the local orientation of collagen fibers. While fiber orientation can easily be determined from SHG intensity images (or even from conventional light microscope images) in tissues with simple structure, such as rat-tail tendon, fibril orientation is often difficult to determine from intensity measurements alone in more complex tissues. Polarized light microscopy and optical coherence tomography (OCT) represent two other techniques that can provide information about collagen orientation in tissues with complex organization. SHG has the advantage that it can be used quantitatively to determine fibril orientation in thick tissue samples to a depth of $\sim 200 \mu \mathrm{m}$ and with an axial resolution comparable to the Rayleigh range of the focused beam. In polarization-modulated SHG microscopy, the information about orientation in the signal stems mainly from the interaction of the 
intense laser light with the collagen fibers in the focal spot (see Chapter 4 for a more detailed explanation of how orientation information is obtained). In polarized light microscopy (or polarization sensitive OCT) information about orientation results from the birefringence of the material the light propagates through. In this case, information about collagen orientation is always integrated over the entire depth through which the light propagates. Birefringence also affects the laser light in SHG microscopy as it propagates to the focal spot; however, we will see that it has no effect on measurement of orientation.

In this chapter, we discuss polarization-modulated SHG measurements (in transmission mode) of collagen orientation in thin samples of tissue in which matrix organization is far more complex than it is in rat-tail tendon. The structural complexities associated with pathological changes in the matrix are investigated in two tissues: cartilage and liver. Polarization-modulated SHG was used in thin tissue sections in transmission mode to analyze organizational changes in collagen related to two pathological conditions-osteoarthritis of human knee cartilage and liver fibrosis in a mouse model. Finally, reflection-mode detection was used in thick samples-porcine skin-to demonstrate that orientation can be measured as a function of depth in a geometry that is suitable for clinical application. 


\section{Measuring orientation in collagenous tissue}

\section{Tissue source}

Rat-tail tendon fascicles were obtained as discussed in Chapter 6. Bovine fascia was obtained from bovine Achilles tendon from commercial sources. Porcine cornea was obtained from a local abattoir. Samples were mounted on glass slides for analysis. Intervertebral disk samples were a kind gift of Jeff Lotz, Ph.D. of the University of California, San Francisco.

\section{Results}

Tissues with increasingly complex fiber orientation patterns were chosen for study, in order to determine if the polarization-modulation technique for measuring orientation could clearly distinguish among these patterns and to see if fiber organization determined using this technique correlated with morphological information obtained by conventional methodologies such as electron microscopy (Eyden and Tzaphlidou, 2001). Rat-tail tendon, with its highly organized, parallel bundles of collagen fibrils, was the first tissue sample imaged. Figure 7 shows a scan across part of an intact rat-tail tendon fascicle; the section scanned lies in the plane perpendicular to the laser beam (the $x-y$ plane; refer back to Figure 2.1). The line segments in the figure are oriented along the local fiber direction. 


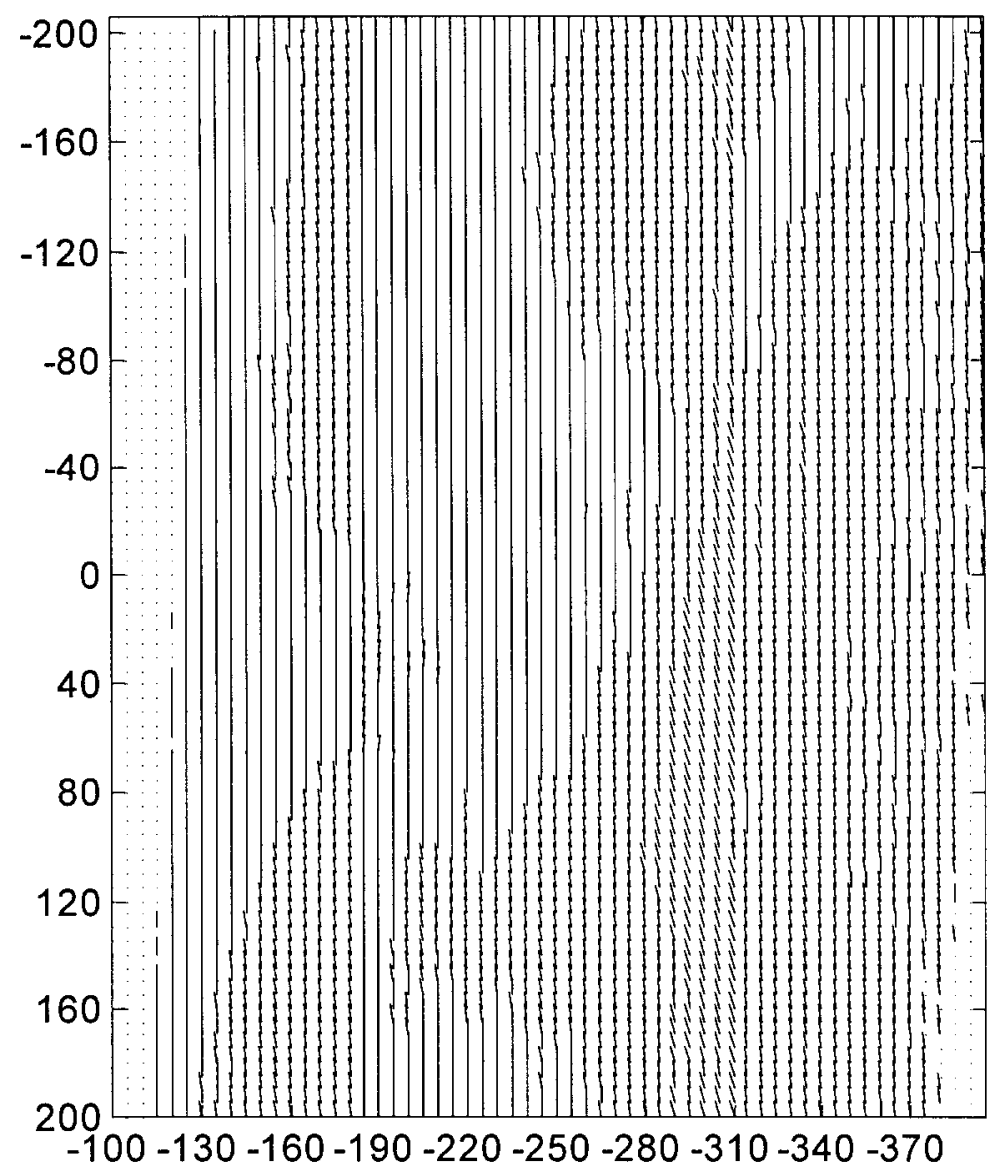

Figure 8.1. Collagen fiber orientation in rat-tail tendon.

Next, a tissue characterized by regular changes in fibrillar orientation-the lamellar rings of intervertebral disks-was imaged. Fibrillar orientation alternates by about 60 degrees (relative to the vertical axis of the spinal cord) in successive lamellar rings. Figure 8.2a shows a visible light micrograph (obtained from the CCD camera) of a $30 \mu \mathrm{m}$ thick frozen section of intervertebral disk. Figure $8.2 \mathrm{~b}$ gives a plot of the orientation data in approximately the same region as shown in the micrograph. Figure $8.2 \mathrm{c}$ is a histogram of fiber orientation, indicating that the difference in orientation between the two regions is approximately 60 degrees. 

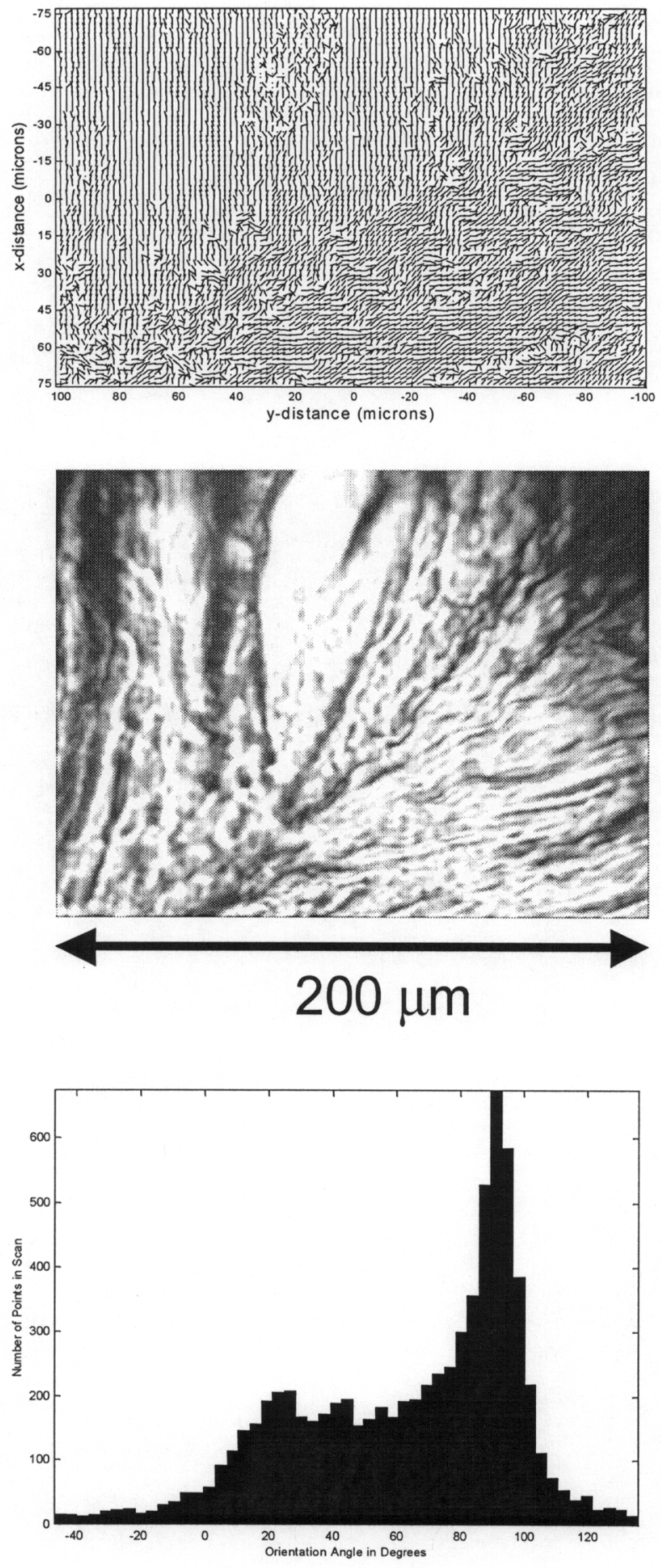

Figure 8.2. (a.) Visible light micrograph of a small region of an $x-y$ section of intervertebral disk showing two different regions of fiber orientation. (b.) Orientation image of approximately the same region. (c.) Histogram showing the frequency of fiber orientation in the intervertebral disk scan shown in (b). Note that there are two peaks in the distribution, separated by approximately 60 degrees. 
Porcine cornea was imaged because of its unique, interwoven collagen structure (Kay, 1988). Figure 8.3 illustrates fiber orientation in a $0.5 \mu \mathrm{m}$ by $0.5 \mu \mathrm{m}$ region near the top surface of a porcine cornea. A pattern of many discrete regions (with a length scale of about $50 \mu \mathrm{m}$ ) of parallel fibers was observed. A similar pattern of discrete regions containing parallel fibers was observed in bovine tendon fascia (Figure 8.4); it is striking that these regions were approximately one twenty-fifth the area of those in cornea.

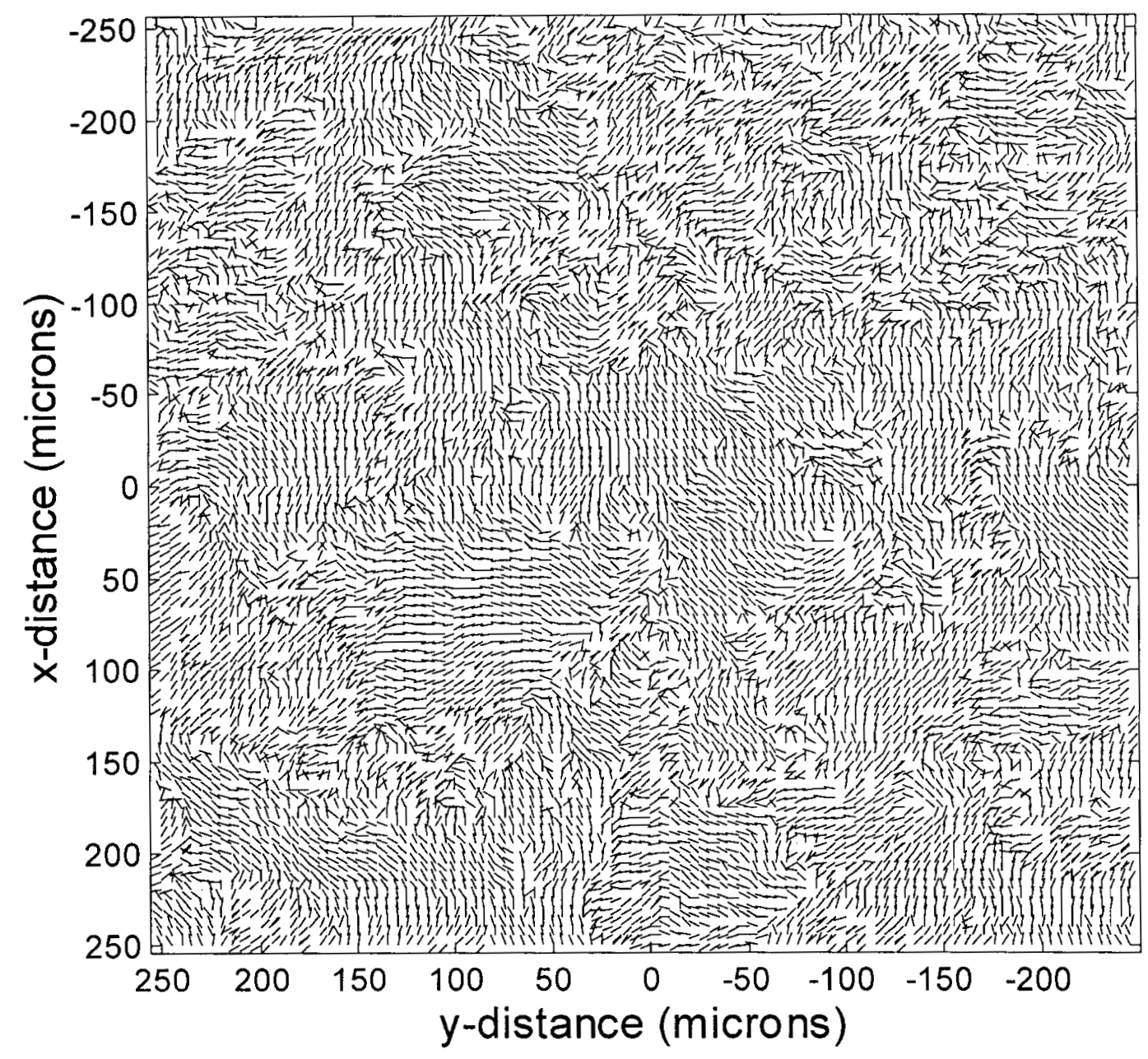

Figure 8.3. Orientation image of an $x-y$ region of porcine cornea. The surface of the cornea was oriented normal to the laser propagation direction. 


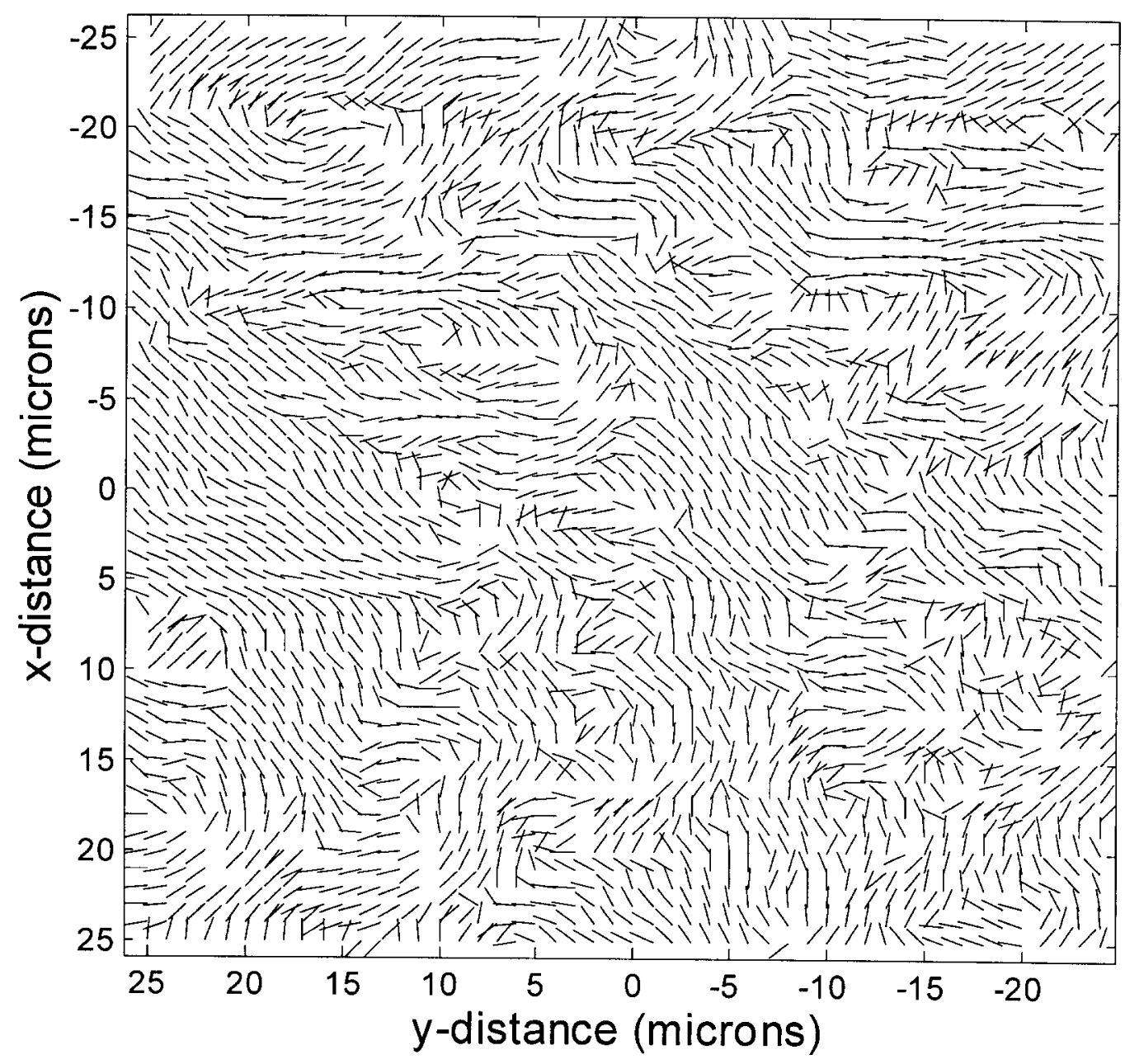

Figure 8.4. Orientation image of an $x-y$ region of bovine tendon fascia measured with a spatial resolution of $1 \mu \mathrm{m}$. Note the similarity between this image and the one in Figure 8.3 - aside from a tenfold change in scale. 


\section{Applications}

\section{Imaging collagen orientation in osteoarth ritic knee cartilage}

\section{Overview and motivation}

Articular cartilage is rarely capable of healing itself. Although full-thickness defects, where damage extends down to the interface with the underlying bone, are repaired spontaneously, the different types of repair tissue that usually form compromise the functionality of the joint and typically degenerate within a year (Hunziker, 1999; Nehrer et. al., 1999). Partial-thickness defects, in which only the cartilage is damaged, do not heal spontaneously at all, except in very young individuals (Athanasiou et. al., 2001). Eventually, damaged cartilage can be destroyed completely, resulting in joints where the bone surfaces are in direct contact, causing severe pain and decreased mobility. The reasons for compromised wound healing in cartilage injury are still poorly understood (Bos et. al., 2001; Goldring, 2000). Given the severity of the long-term consequences, many empirically based approaches to therapeutic intervention are currently under investigation. In recent years, for example, attempts have been made to develop cartilage transplants-either using tissue grafts or cells embedded in a suitable scaffold--to heal localized damage (Hunziker, 1999).

How can one assess the efficacy of such therapies? Ideally, one would like to distinguish between repair tissue that will degenerate over time and repair tissue that will be structurally and functionally equivalent to undamaged cartilage. At present, properties that can be used to distinguish the two have not been fully defined. However, there are a number of properties that clearly distinguish between normal cartilage and degenerative cartilage. For instance, normal cartilage consists primarily of type II collagen, while 
degenerative tissue is predominantly type I collagen (Yasui and Nimni, 1988). Another distinguishing feature is fibrillar organization. Severe osteoarthritis has been characterized by the presence of disorganized collagen fibers (Pfander et. al, 1999). Finally, some studies have shown that there are distinct boundaries between degenerative tissue and normal articular cartilage, indicating a lack of integration between the normal and repaired tissue (Hunziker, 1999).

There is a major need for non-destructive and minimally invasive imaging methods that can assess these properties. Such an imaging technique must also provide adequate resolution to identify even small areas of repair (a few microns). Furthermore, the value of any in vivo technique for clinical application is greatly enhanced by the ability to image beneath the cartilage surface. Recently, investigators have suggested that microscopic MRI, which gives a measure of collagen organization, could be used to study collagen orientation (Xia et. al., 2001); this technique, however, is limited to a resolution of $10 \mu \mathrm{m}$ and provides only indirect information about collagen fiber orientation. Optical coherence tomography has also been suggested as a potential technique for imaging cartilage in vivo and as a function of depth (Hermann et. al., 1999). However, even polarization dependent optical coherence tomography can only provide indirect information about fiber orientation that is integrated over the depth of the tissue.

As demonstrated above, polarization-modulated SHG can be used to measure collagen orientation. In this work, this technique is applied to the analysis of normal and degenerative articular cartilage from human subjects. 


\section{Tissue source}

Articular cartilage was obtained from the femoral condyles of patients undergoing knee replacement surgery for grade IV degenerative osteoarthritis. This protocol was performed with the informed consent of the patients. Samples were stored at -80 degrees C until analysis.

\section{Immunohistochemical staining}

Maggie Chiu of the Department of Pathology at the University of California, Davis performed the immunohistochemical staining of the cartilage sections, using the following protocol:

An avidin-biotin complex $(\mathrm{ABC})$ peroxidase system was used for immunohistochemical staining. Paraffin-embedded tissue sections $(3-4 \mu \mathrm{m})$ were applied to Fisherbrand Superfrost/Plus microscope slides (Fisher Scientific, Pittsburgh, PA). Tissue sections were deparaffinized in xylene, dehydrated in absolute ethanol, and incubated in 3\% hydrogen peroxide in absolute methanol for 10 minutes at room temperature in order to quench endogenous peroxidase activity. The sections were then digested with $0.05 \%$ trypsin (Type II, T-8128, Sigma Chemicals, St. Louis, MO), pH 7.8 at 37 degrees C for 10 minutes. Nonspecific background staining was blocked by incubating the sections for 30 minutes at room temperature with $10 \%$ heat-inactivated normal horse serum. The solution was then blotted off the slides and incubated with monoclonal antisera against type I collagen (Ab-1) and type II collagen (Ab-2) (Oncogene Research Products, Boston, MA) at 1:600 for 60 minutes at room temperature. Following this incubation and each subsequent incubation, the sections were rinsed 5 minutes in phosphate buffered saline (PBS). The sections were then incubated for 60 minutes at room temperature with a 1:200 dilution of secondary antibody, biotinylated horse anti-mouse immunoglobulin (Vector, Burlingame, CA), followed by incubation for 30 minutes at room temperature with Vectastain R.T.U. Elite $\mathrm{ABC}$ reagent (Vector, Burlingame, CA). Finally, the sections were developed with diaminobenzidine (DAB), rinsed in tap water, counterstained 
with Mayer's hematoxylin, and coverslipped with a mounting medium. All incubations were performed in a humidified chamber. Formalin-fixed placenta (for type I collagen) and chondrosarcoma (for type II collagen) were used as positive controls; PBS was substituted for the primary antibody as a negative control.

SHG scans were performed on the same sections that had been immunohistochemically stained; even serial sections differed too greatly from each other to overlay SHG data obtained in one section on images of another section that had been immunohistochemically stained. The effects of the staining procedure on the measurement of orientation was studied; staining with either type I or type II antibodies had no detectable effect.

Results

Normal articular cartilage

Hematoxylin and eosin staining as well as immunohistochemical analysis of normal articular cartilage was consistent with previous reports (Yasui and Nimni, 1988). The surface layer and columnar layer can be clearly seen (Figure 8.5). As anticipated, antibody staining revealed the presence of type II collagen exclusively; type I collagen was not present (data not shown). Fibrillar orientation data obtained using polarizationmodulated SHG in an unstained section are shown in Figure 8.6. The SHG image clearly shows the expected surface region where collagen fibers run parallel to the surface (along the $\mathrm{x}$-axis in our coordinate system) and the columnar layer beneath it where the fibers run approximately normal to the surface. The highly organized nature of the collagen fibers is clearly evident. 

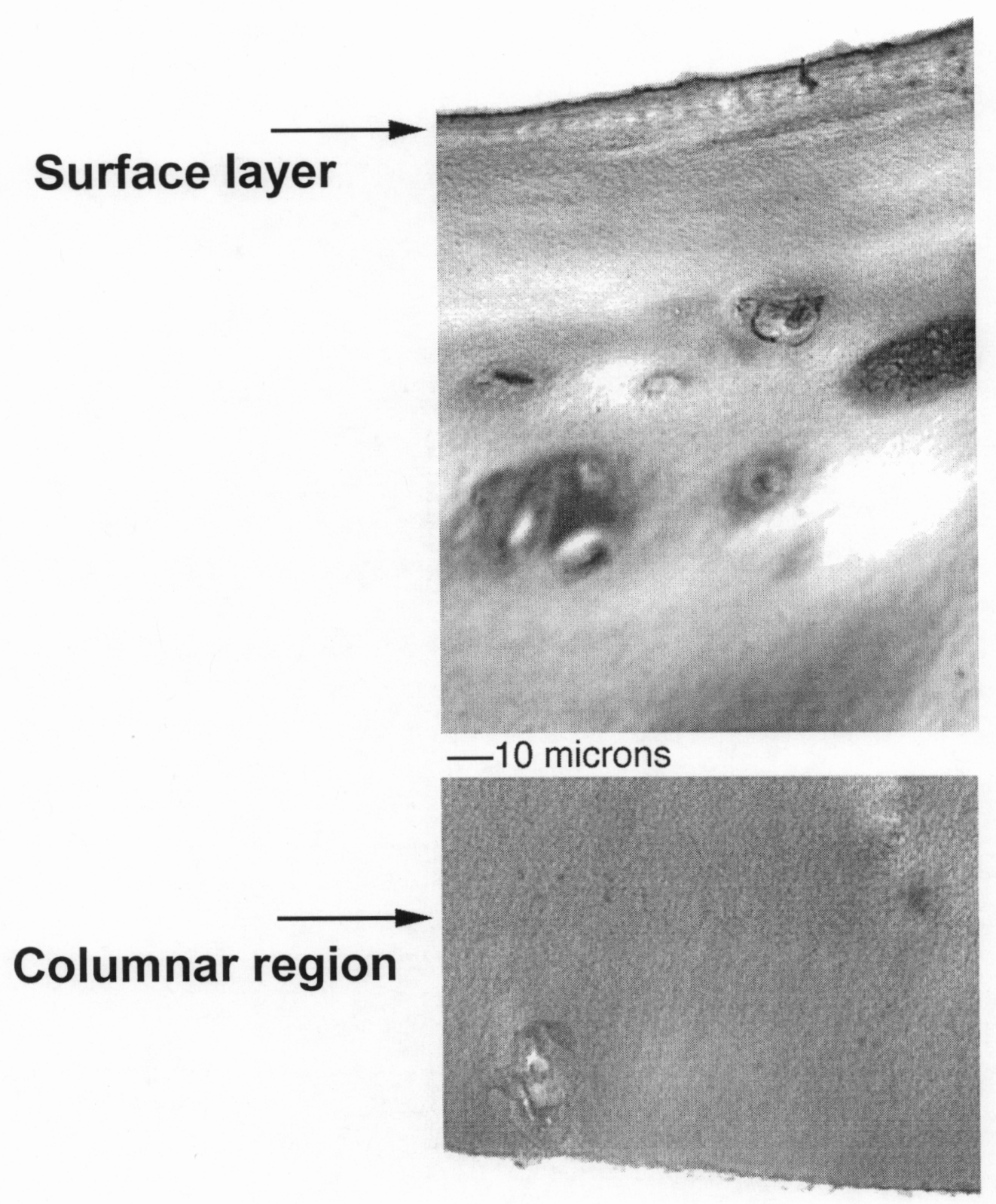

Figure 8.5. Photomicrograph of a section of normal cartilage from the femoral condyle, stained with H\&E, near the top of the section (surface layer) and near the bottom of the section (columnar region). 


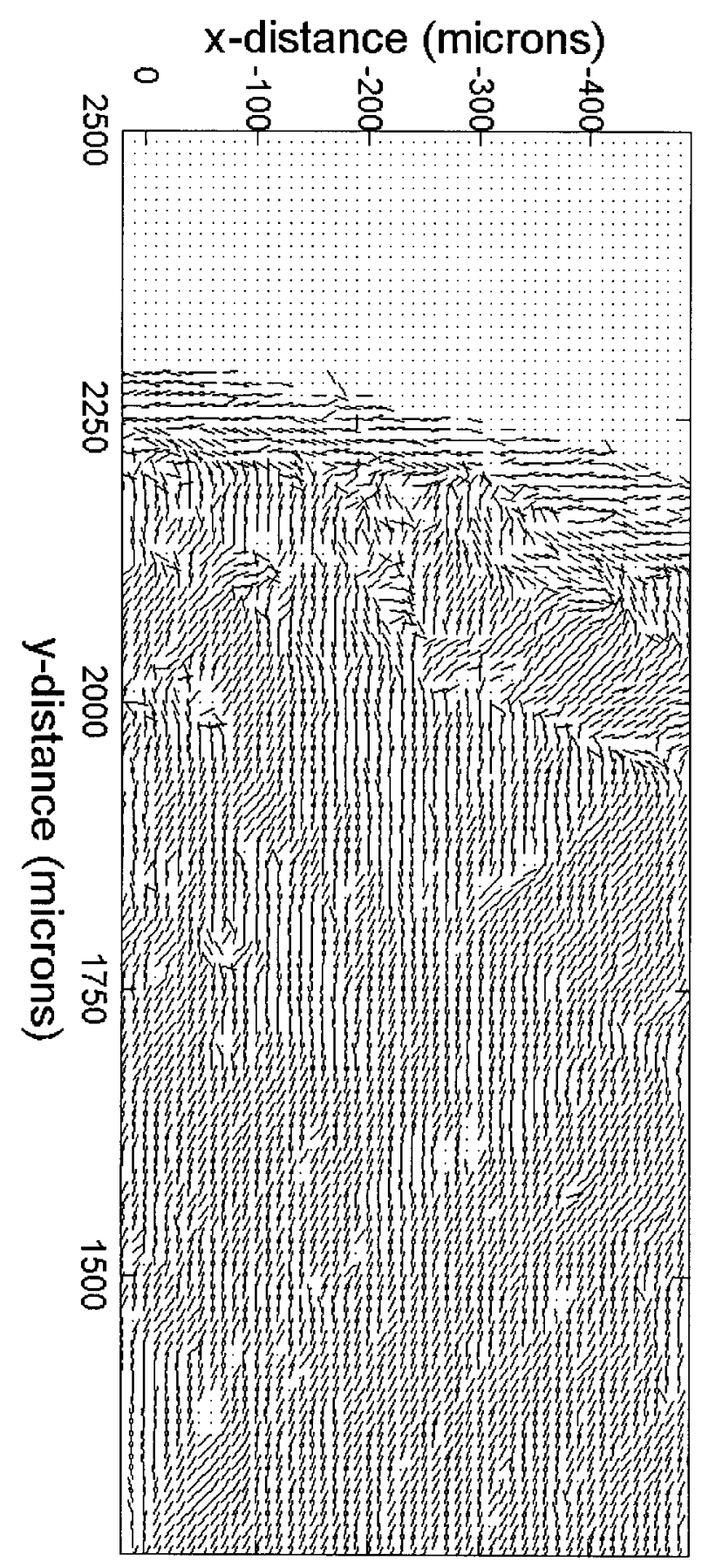

Figure 8.6. SHG orientation image of an unstained cross section of normal cartilage from the femoral condyle. Each line segment corresponds to the collagen fiber orientation measured at that point; all line segments are drawn to equal length. The top surface of the cartilage (top of the figure) runs approximately parallel to the $\mathrm{x}$-axis, while the $y$-axis represents depth. The absence of orientation line segments indicates weak or no SHG signal. 


\section{Degenerative tissue}

Sections of articular cartilage exhibiting different levels of degeneration were imaged. Figure 3 shows both SHG and photomicrographic images from the same region in the sample. In this sample the surface layer was not imaged. Note that the region of low SHG intensity (Figure 8.7a) corresponds to the region of high type I staining (Figure $8.7 \mathrm{~b}$ ) and low type II staining (Figure $8.7 \mathrm{c}$ ). There are several areas where the tissue is stained with type II antibodies (and not with type I antibodies), but where there is also weak SHG signal-for instance, near the top, right corner of the images. The signal is strong in a band near the left edge of the sample that is stained with both type I and type II antibodies. It should be emphasized that the degree of type II staining is not uniform, even in those regions that are unstained using type I antibodies.

In Figure 8.8, the image of the collagen fiber orientation was overlaid on the photomicrograph of the section stained for type I collagen (shown above in Figure 8.7b). A large region at the lower right (indicated by "A") shows highly organized, parallel fibers corresponding to the region of type II collagen staining. There are marked changes in orientation in the fibers corresponding to the areas of type I collagen staining. In some cases, the fibers are faint (corresponding to weak signal) and randomly organized ("B"). In other areas, the fibers have some degree of parallel orientation which differs markedly from that of the fibers in the type II region ("C"). Note that in the region just below and to the right of the band of type I stained tissue there is an area of disordered fibers that corresponds to a region faintly stained with both type I and type II ("D"). 


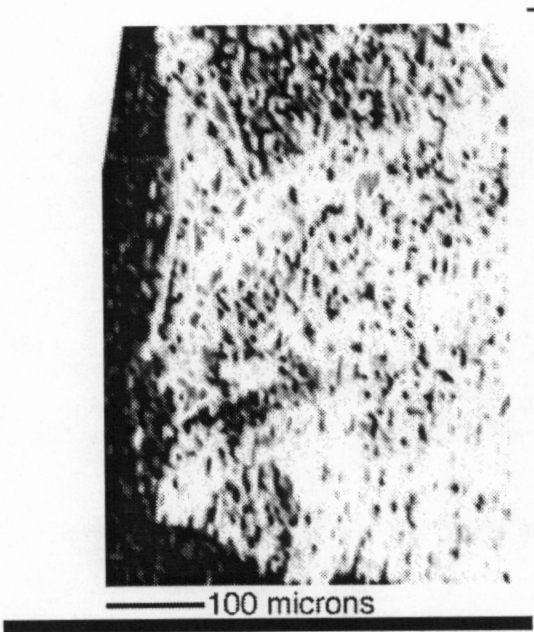

a

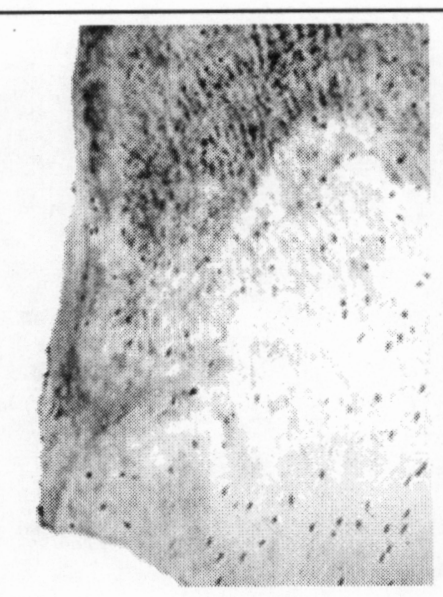

b

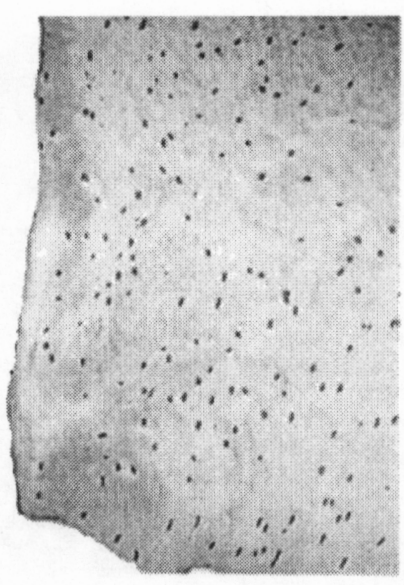

c

Figure 8.7. (a.) SHG intensity image of a cross section of damaged cartilage; the section was stained using type I collagen antibodies. (b.) Same as in (a), but the section was stained using type II collagen antibodies. (c.) Visible light photomicrograph of the section imaged in (a). (d.) Visible light photomicrograph of the section imaged in (b).

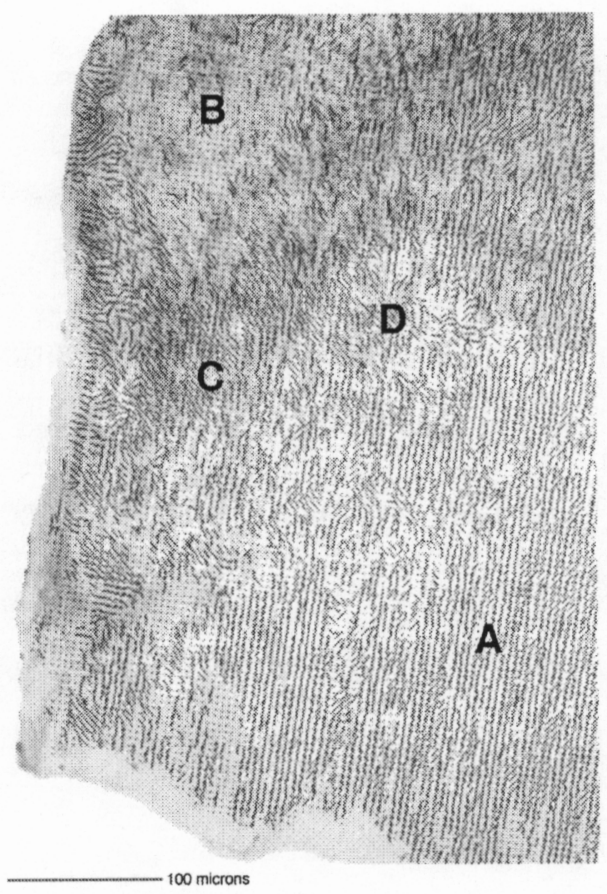

Figure 8.8. Visible light photomicrograph of cross section of damaged cartilage stained using type I collagen antibodies, overlaid with an orientation image obtained from the SHG scan. 
Similar results were obtained from a sample of knee cartilage from a second subject, shown in Figures 8.9 and 8.10. The second harmonic signal (Figure 8.9a) is generally weaker in the region where the tissue was strongly stained with type I antibodies (Figure 8.9b); interestingly, there is a region near the middle of the stained area where relatively strong SHG is observed. In Figure 8.10 there is a clear change in fibrillar orientation between the region corresponding to type II collagen (Figure 8.9c) and the region corresponding to type I collagen. In this sample, the collagen in the type II region has a high degree of fibrillar orientation all the way to the edge of the type I region ("A"). Fibrillar orientation in the region of type I staining was highly disordered throughout ("B"). The surface region, characterized by fibrils oriented parallel to the surface, can clearly be seen at the top of the figure ("C"). In a few areas adjacent to the type I staining and in areas stained with both type I and type II, there appears to be some mild disorder ("D").

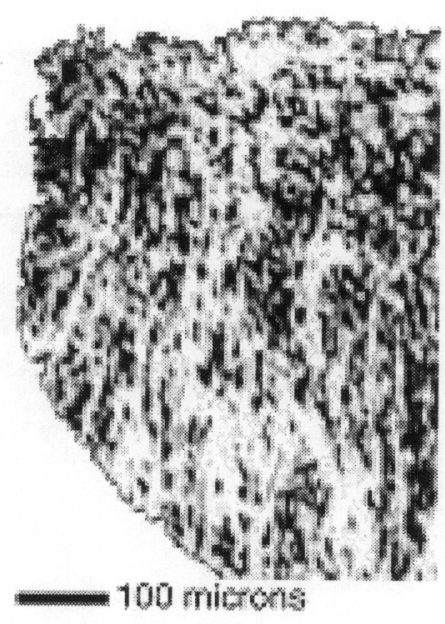

a

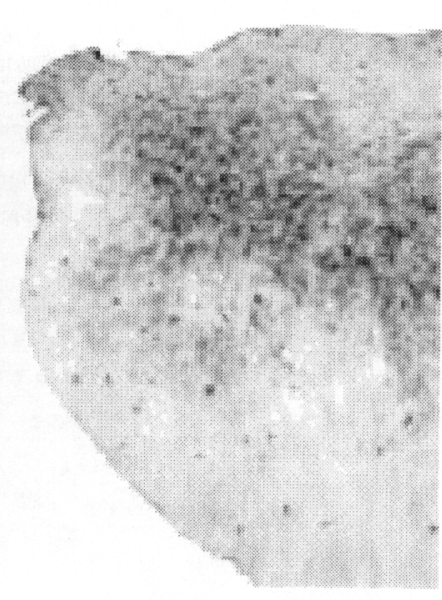

b

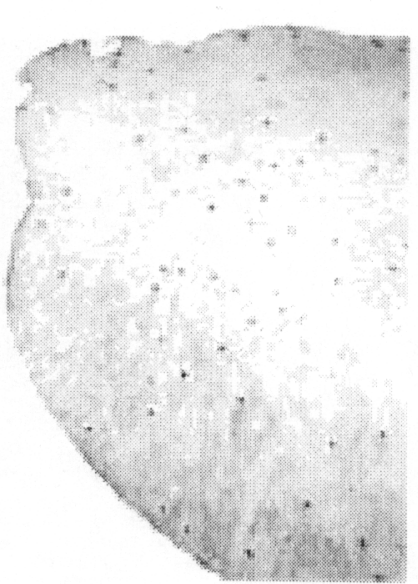

c 
Figure 8.9 (a.) SHG intensity image of a cross section of damaged cartilage; the section was stained using type II collagen antibodies. (b.) Visible light photomicrograph of the same cartilage sample as used to obtain (a), but using type I collagen antibodies for staining. (c.) Visible light photomicrograph of the section imaged in (a).

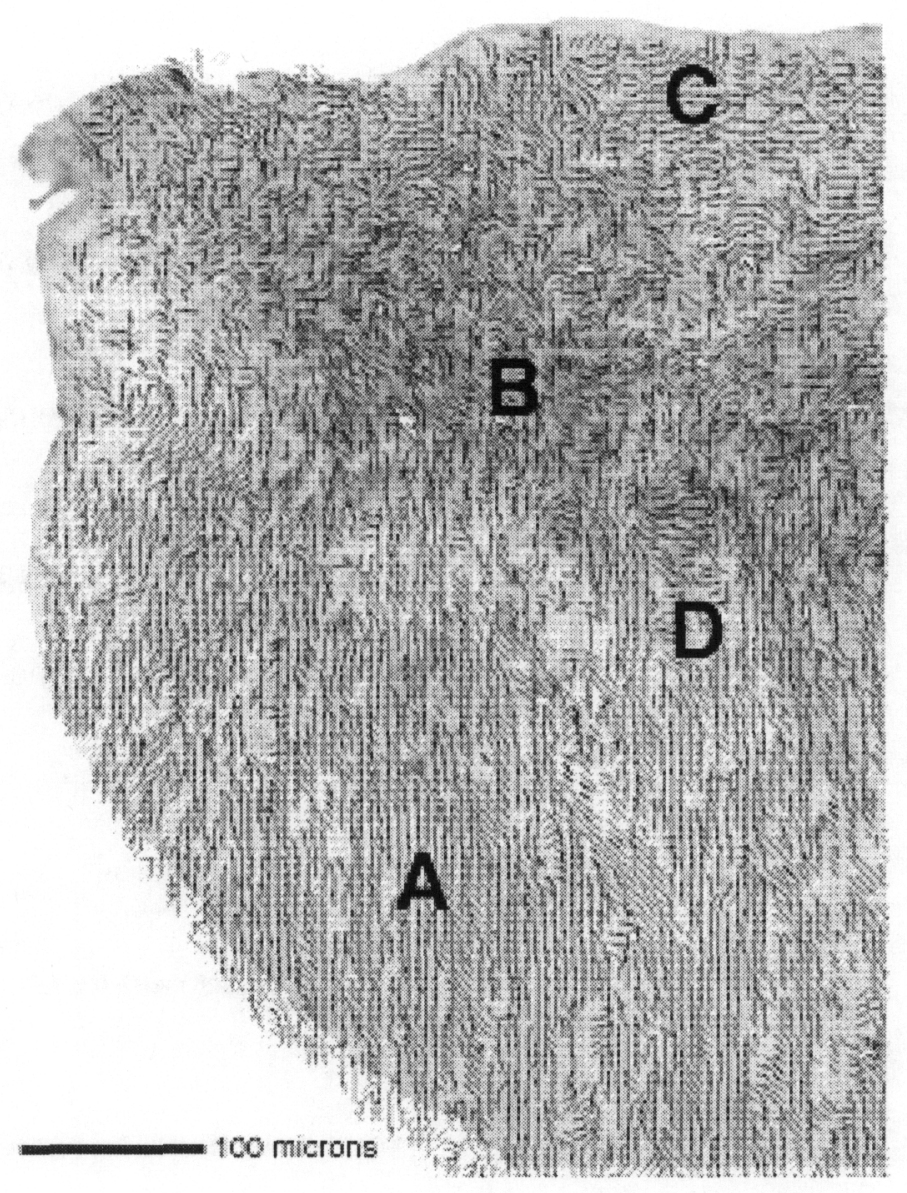

Figure 8.10 Visible light photomicrograph of cross section of damaged cartilage stained using type I collagen antibodies, overlaid with an orientation image obtained from the SHG scan.

In a cartilage section from a third subject, a large region containing a mixture of type I and type II collagen was observed (Figures 8.11 and 8.12). The SHG intensity (Figure 8.11a) shows a marked decrease corresponding to the areas of darkest type I antibody staining. The distribution of type I collagen as assessed by antibody staining was variable (Figure $8.11 \mathrm{~b}$ ). Three distinct areas of intense staining are visible below the 
surface; there is a diffuse pattern of type I staining throughout almost the entire section. Type II collagen is primarily present in the lower part of the section, inter-mixed with type I collagen. Highly organized, parallel collagen fibers are seen across the top of the section ("B"); however, unlike normal collagen, they are oriented neither parallel nor perpendicular to the surface. There are a few areas where fibers are oriented normal to the surface ("B"); however, the bulk of the section consists of a pattern of extremely disorganized fibers ("A" and "C").

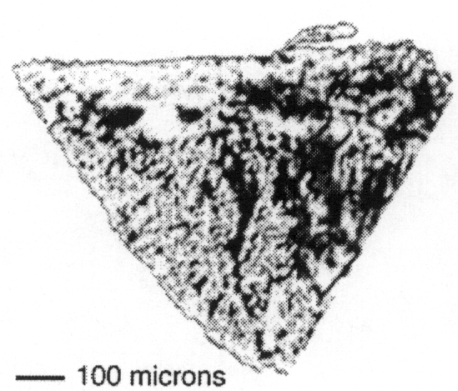

a

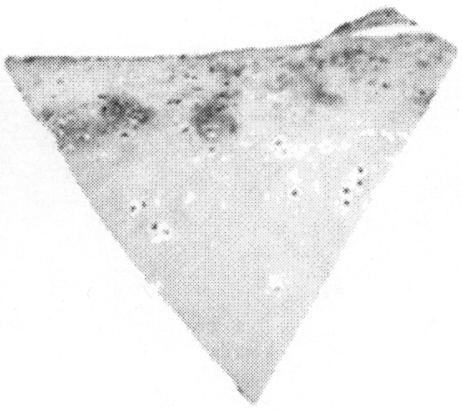

b

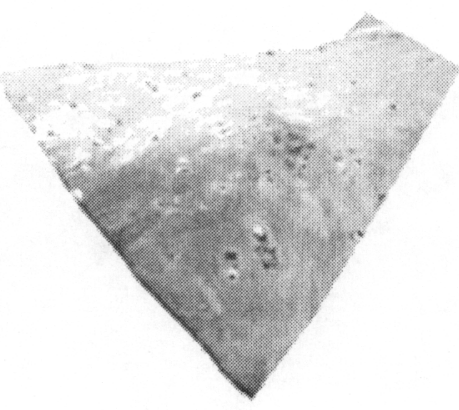

c

Figure 8.11 (a.) SHG intensity image of a cross section of damaged cartilage; the section was stained using type II collagen antibodies. (b.) Visible light photomicrograph of the same cartilage sample as used to obtain (a), but using type I collagen antibodies for staining. (c.) Visible light photomicrograph of the section imaged in (a). 


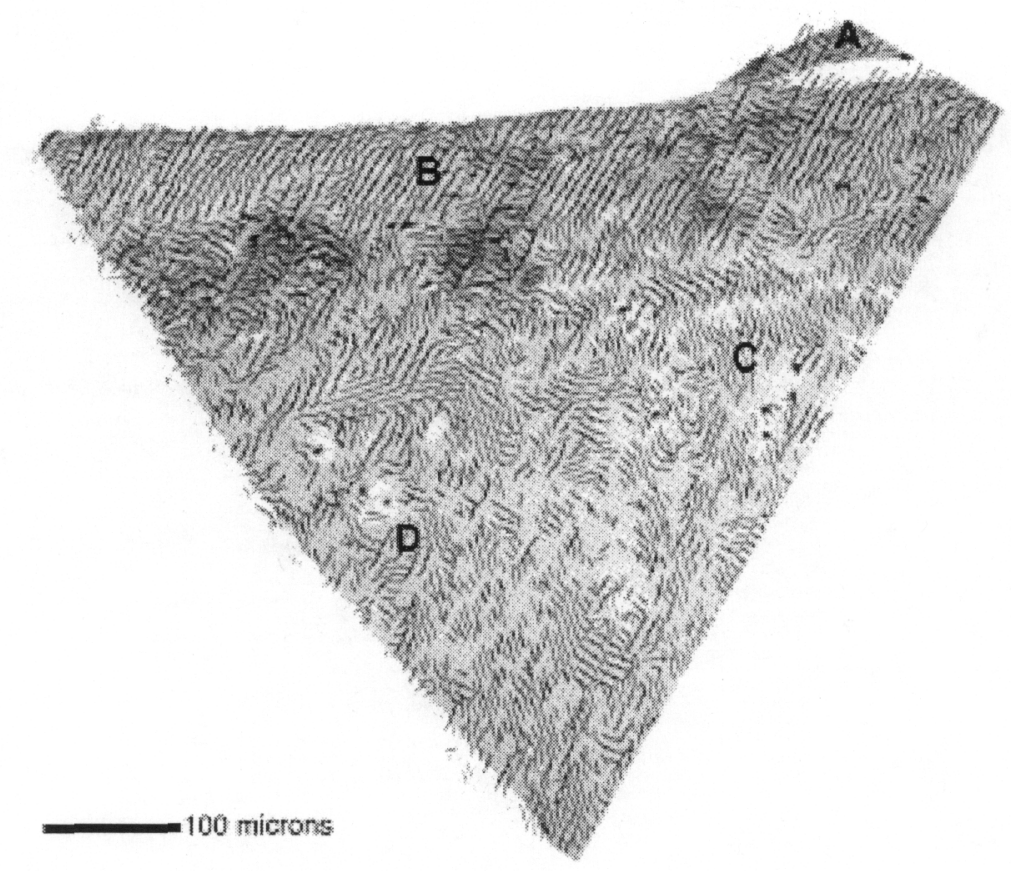

Figure 8.12. Visible light photomicrograph of cross section of damaged cartilage stained using type I collagen antibodies, overlaid with an orientation image obtained from the SHG scan.

\section{Discussion}

In this study, SHG microscopy has been used to analyze damaged articular cartilage obtained at surgery from subjects with degenerative osteoarthritis. Clearly, polarization-modulated SHG is a useful technique for determining the degree of tissue organization. As many other investigators have reported, the presence of type I collagen was observed in the areas of degeneration in articular cartilage. In some cases, there were clear distinctions between areas of type I collagen and type II collagen, while in other samples both types of collagen were present in the same area. SHG analysis allowed examination of fiber orientation in these same samples. Normal cartilage, which consists solely of type II collagen, was characterized by a highly organized pattern of fiber orientation. The characteristic pattern of collagen organization in cartilage was 
observed: fibers in the surface region were seen to run parallel to the surface, while those deeper in the tissue were oriented perpendicular to the surface. Fiber organization in the degenerative cartilage showed several patterns observed histologically by previous investigators (Nehrer et. al., 1999). For example, in one sample, fibers in parts of the region stained for type I collagen had parallel organization, but at a markedly different angle than in the adjacent normal type II collagen. In other areas of type I collagen, the fibers were randomly oriented with no discernible pattern. It seems likely that the bundles of parallel fibers correspond to areas of reparative tissue sometimes referred to as fibrous tissue or fibrocartilage. The areas of highly disorganized fibers are most likely examples of what has been termed fibrous spongiform tissue (Nehrer et. al., 1999). The areas of mixed type I and type II collagen, which showed predominantly randomly oriented fibers, most likely represent a transitional zone. These areas are characterized by varying degrees of disorder.

The intensity of the SHG signal did not correlate as well with the patterns of degeneration as did fiber orientation imaging. However, there was some degree of correlation between intensity and parallel fiber orientation, particularly that of type II collagen. In one case, in several regions of intense type I staining, virtually no SHG signal was observed. The intensity appeared to be better correlated with fiber orientation than with collagen type.

The results of this study suggest that SHG imaging may be a useful tool for distinguishing between normal articular cartilage and different stages of degenerative tissue formation. Resolution was sufficient to detect areas of abnormal cartilage as small as a few microns. 


\section{Imaging liver fibrosis}

The formation of scar tissue is a natural response of the body to injury. Scar tissue-consisting predominantly of type I collagen—can form rapidly and prevent further damage; remodeling of the scar tissue over time can often restore function to the damaged tissue. Fibrosis is a condition characterized by the deposition of excessive type I collagen. In liver, exposure to toxic substances or diseases such as hepatitis can lead to a fibrotic response to tissue damage. Certain cells in the liver ("stellate" cells) begin to produce collagen fibrils that spread through the liver in a pattern that depends on the cause of fibrosis. Neubauer et. al. (2001) presents an extensive review of the current understanding of liver fibrosis.

Current techniques for imaging liver fibrosis require staining of tissue sections, typically using Masson's Trichrome, which stains collagen blue. Polarization-modulated second harmonic generation can be used to image unstained sections of fibrotic liver tissue. In this work, fiber orientation was imaged in sections of tissue obtained from liver fibrosis in a mouse model in order to determine whether SHG imaging provided information comparable to that obtained by looking at stained sections of tissue. 
Tissue source

Murine liver tissue was provided by Mark Zern M.D. of the University of California, Davis. Severe fibrosis was caused by exposing the mice to carbon tetrachloride $\left(\mathrm{CCl}_{4}\right)$. Both control and severely fibrotic liver tissue was studied.

\section{Results and Discussion}

Figure $8.13 \mathrm{a}$ shows photomicrograph of a stained section of liver tissue from a control mouse. The collagen orientation image obtained in an unstained, serial section is shown in Figure 8.13b. Observe that the collagen in this healthy tissue is confined to the arterial wall.

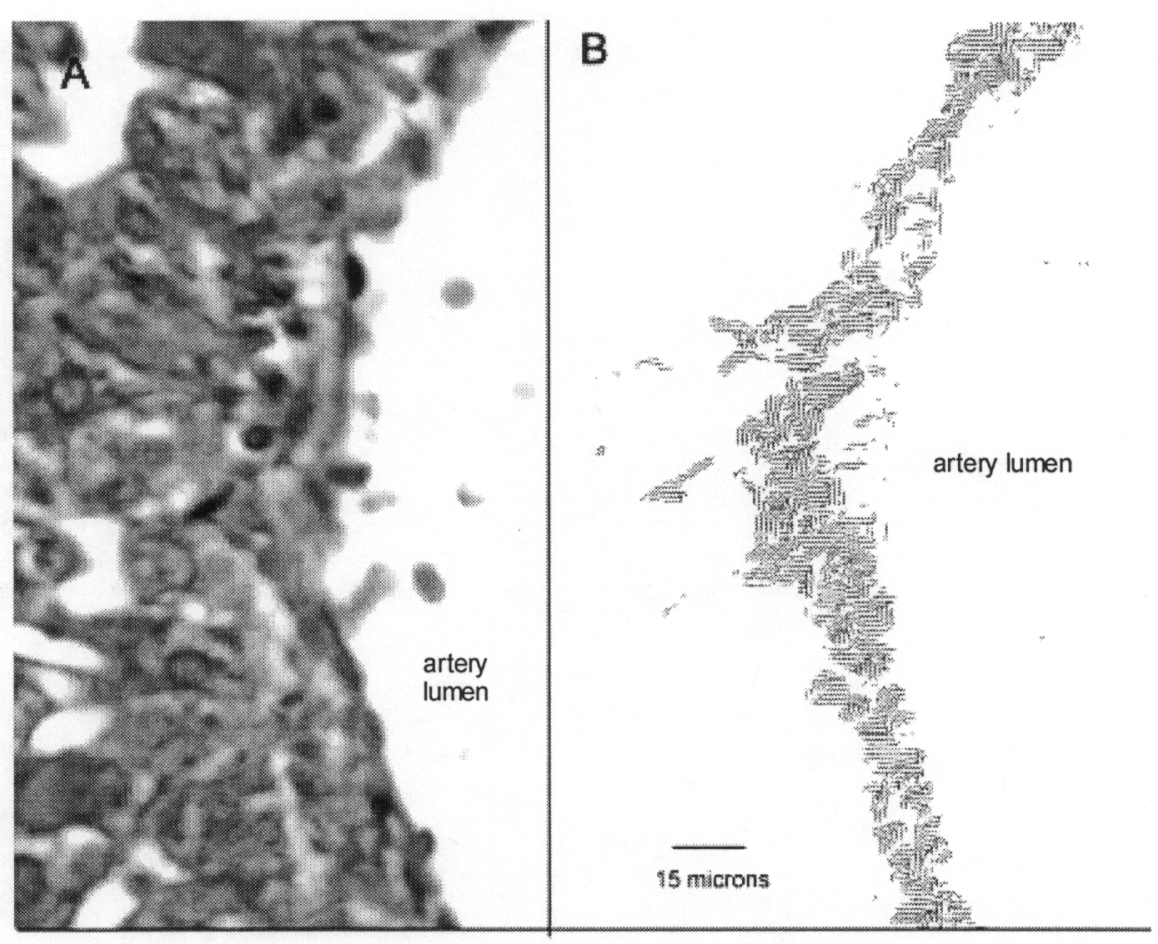

Figure 8.13. (a) Photomicrograph of control liver tissue near an artery stained using Masson's Trichrome (b) SHG orientation imaging of corresponding region of tissue in an unstained, serial section. 
In Figure 8.14a, a photomicrograph of a section of severely fibrotic liver tissue is shown. Figure $8.14 \mathrm{~b}$ shows a corresponding SHG orientation image obtained in an unstained serial section. As expected, polarization-modulated SHG imaging provides information about collagen fibril orientation that cannot be obtained from the photomicrograph of the histologically stained section. Surprisingly, SHG microscopy detects collagen in regions where none can be observed in the stained section. This can be readily seen in the bottom, center of Figure 8.14a (arrow); the circular collagen structures that are clearly seen in the SHG image are not seen in the stained section.
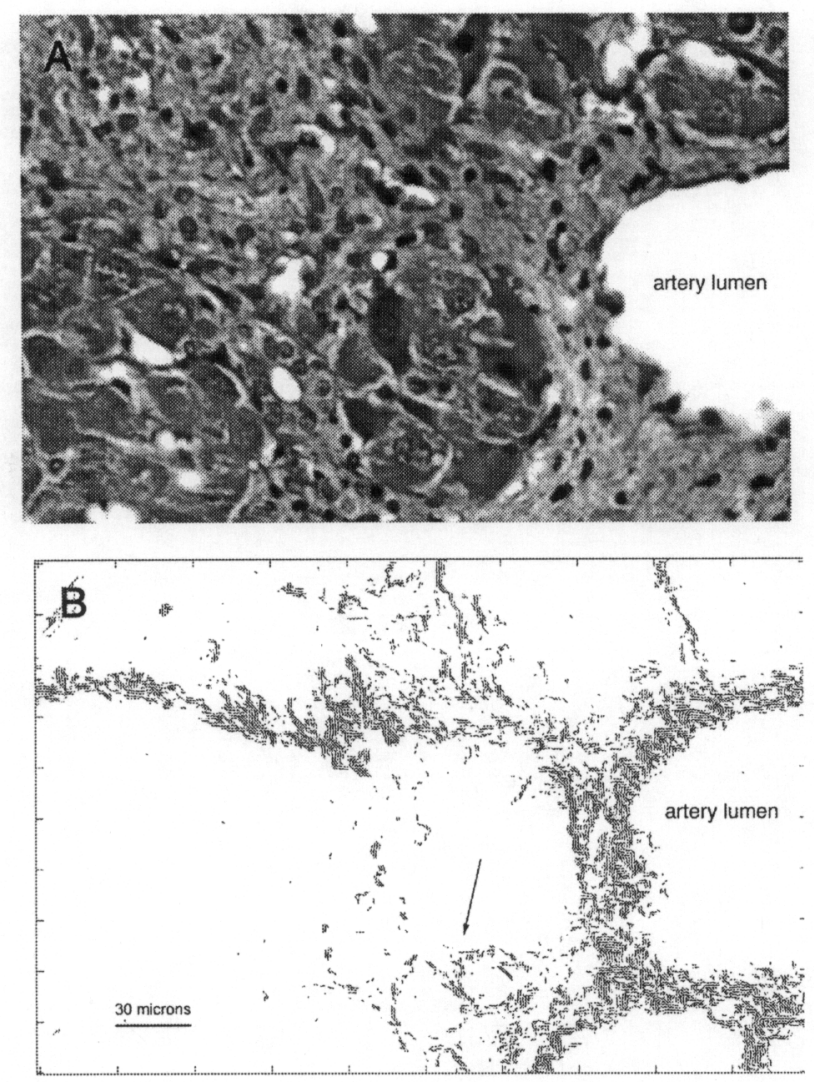

Figure 8.14. (a) Photomicrograph of severely fibrotic liver tissue near an artery stained using Masson's Trichrome (b) SHG orientation imaging of corresponding region of tissue in an unstained, serial section. 


\section{Measuring orientation in thick samples (reflection mode)}

The measurements of orientation in collagen presented above were made using transmission-mode detection in relatively thin tissue samples or in thin sections of tissue. Since second harmonic light is generated predominantly in the forward direction, transmission-mode detection provides a strong signal; its use has greatly facilitated the initial studies of collagen orientation described above. However, most tissue is highly scattering and thus significant second harmonic light is back-scattered, allowing reflection-mode SHG microscopy. Orientation measurements at depths of up to $\sim 200 \mu \mathrm{m}$ in tissue were made using reflection mode detection.

Polarization-modulated SHG microscopy was applied to imaging collagen fibril orientation in porcine skin, a tissue which is very similar to human skin. Signal was detected only in reflection mode; the tissue was too thick $(\sim 1 \mathrm{~cm})$ to obtain any signal in transmission mode. Figure 8.15 illustrates collagen fibril orientation in planes at different depths within the tissue. Orientation information can be obtained at depths of up to $\sim 200$ $\mu \mathrm{m}$; at greater depths, the signal strength, even at the maximum laser power available from the system, is too weak. 

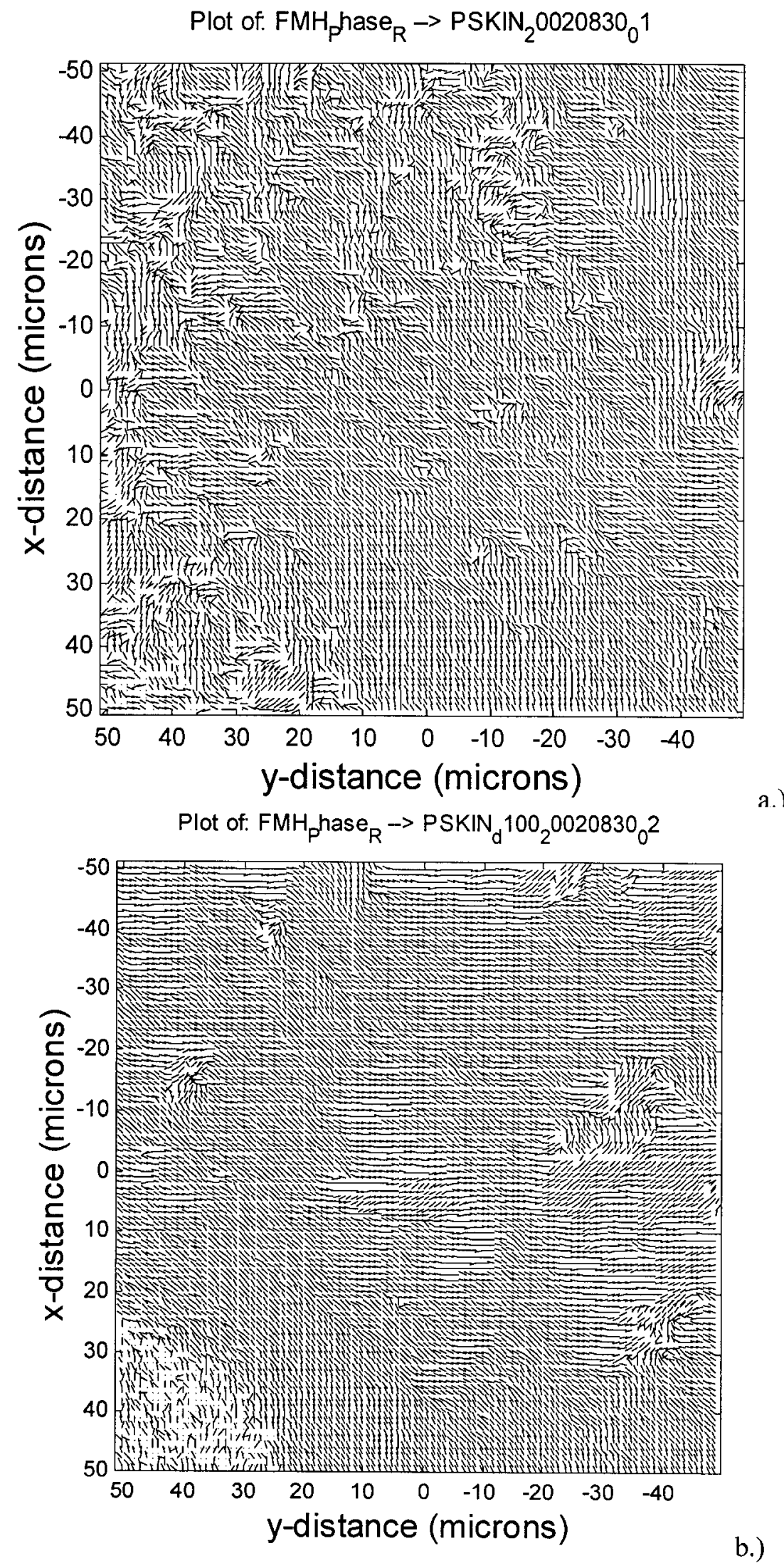


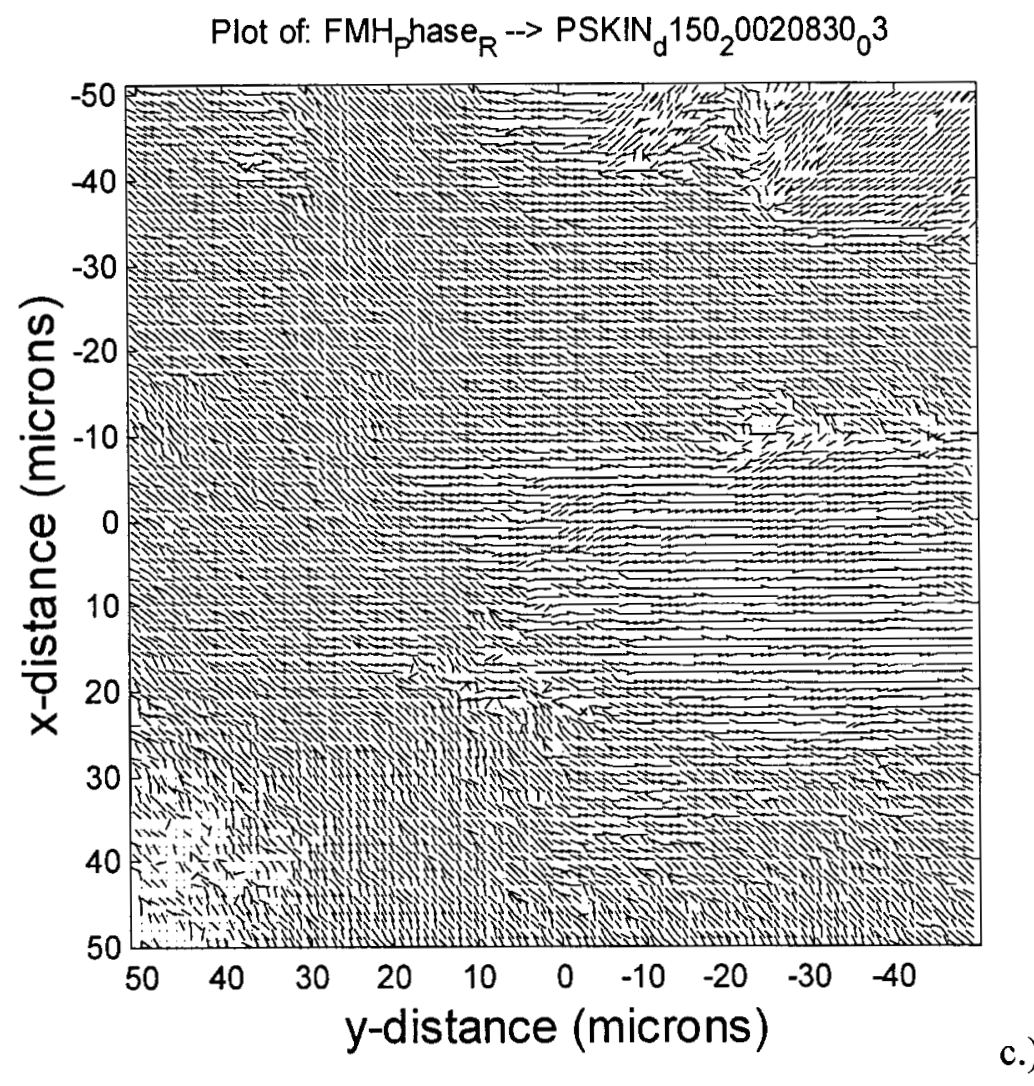

Figure 8.15. Images of collagen orientation at different depths within a sample of porcine skin. (a). Surface. $z=0 \mu \mathrm{m}$. (b.) $z=150 \mu \mathrm{m}$ (c.) $z=225 \mu \mathrm{m}$. Note that depth values have been calculated assuming an index of refraction in porcine skin of $n=1.5$. The laser intensity used to obtain images (b.) and (c.) was double that used to obtain image (a.). 


\section{References}

K. A. Athanasiou, A. R. Shah, R. J. Hernandez, R. G. LeBaron. 2001. Basic science of articular cartilage repair. In Schenck RC, Miller MD, Eds. Clinics in Sports Medicine: Osteochondral Injuried of the Knee. 20:223-247.

P. K. Bos, J. G. V. M. van Osch, D. A. Frenz, J. A. N. Verhaar, H. L. Verwoerd-Verhoef. 2001. Growth factor expression in cartilage wound healing: temporal and spatial immunolocalization in a rabbit auricular cartilage wound model. Osteoarthritis and Cartilage. 9:382-389.

B. Eyden and M. Tzaphlidou. 2001. Structural variations of collagen in normal and pathological tissues: role of electron microscopy. Micron. 32:287-300.

M. B. Goldring. 2000. The role of the chondrocyte in osteoarthritis. Arthritis and Rheumatism. 43:1916-1926.

E. B. Hunziker. 1999. Articular cartilage repair: are the intrinsic biological constrains undermining this process insuperable? Osteoarthritis and Cartilage. 7:15-28.

J. M. Hermann, C. Pitris, B. E. Bouma, S. E. Boppart, C. A. Jesser, D. L. Stamper, J. G. Fujimoto, M. E. Brezinski. 1999. High resolution imaging of normal and osteoarthritic cartilage with optical coherence tomography. Journal of Rheumatology. 26;627-635.

S. Nehrer, M. Spector, T. Minas. 1999. Histologic analysis of tissue after failed cartilage repair procedures. Clinical Orthopaedics and Related Research. 365:149-162.

K. Neubauer, B. Sailee, G. Ramadori. 2001. Liver fibrosis and altered matrix synthesis. Canadian Journal of Gastroenterology. 15:187-193. 
D. Pfander, R. Rahmanzadeh, E. E. Scheller. 1999. Presence and distribution of collagen II, collagen I, fibronectin, and tenascin in rabbit normal and osteoarthritic cartilage. Journal of Rheumatology. 26:386-394.

Y. Xia, J. B. Moody, N. Burton-Wurster, G. Lust. 2001. Quantitative in situ correlation between microscopic MRI and polarized light microscopy studies of articular cartilage. Osteoarthritis and Cartilage. 9:393-406.

N. Yasui and M. E. Nimni. 1988. Cartilage Collagens. In Nimni, ME, Ed. Collagen: Biochemistry, Vol. 1. Boca Raton, FL: CRC Press. 225-242. 


\section{CONCLUSION}

Techniques capable of imaging collagen structure, especially those that can be used in vivo, have important potential applications in understanding the fundamental biology of pathological conditions related to changes in collagen. Second harmonic generation microscopy using ultra-short pulse lasers can be used to image biological tissue in vivo. This technique has an important advantage over other in vivo techniques for studying collagen—it provides orientation information and structural information at high resolution $(\sim 1 \mu \mathrm{m})$ and as a function of depth. This work has presented a new technique-polarization-modulated SHG microscopy—and applied it to studying the nonlinear optical properties of collagen. Polarization-modulated SHG microscopy can be used to determine simultaneously the orientation of collagen fibrils, to measure a parameter $\gamma$ related to the ratio of the two independent elements in the second order nonlinear susceptibility tensor, and to measure the strength of the second harmonic signal generated in collagen fibrils independent of their orientation (Table 9.1).

SHG microscope images of collagen—even collagen in highly-organized, uniform samples such as thin sections of rat-tail tendon-show strong fluctuations in signal strength as a function of transverse position. This work confirms earlier suggestions that this fluctuation is due to the fact that neighboring fibrils may be oriented parallel to each other or anti-parallel to each other. Using different microscope objectives-and a new technique that uses a quartz plate to calibrate the signal from all of them--permits measurement of the scale on which fibrils are oriented parallel or antiparallel. Regions of parallel orientation are less than $1.5 \mu \mathrm{m}$ in diameter. This suggests that only a few large-diameter collagen fibrils, oriented parallel to each other through 
random fluctuations, can account for increases in second harmonic signal. Because electron microscopy studies show that collagen fibrils are oriented randomly parallel and anti-parallel to their neighbors in a host of tissues (not just tendon), fluctuation in the second harmonic signal make it difficult to use the magnitude of the signal to detect changes in collagen structure.

The polarization-dependence of the SHG signal, on the other hand, varies much less with position in a sample. The effect on SHG polarization dependence of several important, biologically relevant changes in collagen structure was investigated: nonenzymatic glycation and crosslinking, thermal denaturation, enzymatic digestion, and dehydration. Complete enzymatic digestion and complete thermal denaturation, not surprisingly, resulted in loss of SHG signal; no change in polarization dependence was observed prior to this point. Dehydration of collagen significantly affected the polarization dependence of SHG; this suggests that the highly polarizable water molecules found in collagen structures play an important role in determining the second order nonlinear susceptibility. Most surprisingly, increased glycation and nonenzymatic crosslinking - both of which lead to significant changes in collagen structure- did not result in a detectable change in the polarization dependence of the SHG signal. This suggests that the organization of collagen into fibrils and the interaction of these fibrils with surrounding water molecules is the key property of collagen that determines its second order nonlinear susceptibility.

The most promising results-- from the point of view of developing a diagnostic tool-in this study were obtained from measurements of collagen fibril orientation. In two tissues that had been modified by pathological conditions that affect collagen 
structure-osteoarthritic knee cartilage and fibrotic liver tissue-a significant change in the presence or absence of collagen and in the pattern of collagen orientation was observed using polarization-modulated SHG. Because this technique allows the measurement in reflection-mode of collagen orientation as a function of depth in tissue (to a depth of a few hundred microns), it could potentially be applied to in vivo imaging designed to diagnose skin diseases, such as melanoma or abnormal wound healing, by detecting changes in collagen organization.

Table 9.1. Measurements made using polarization-modulated SHG microscopy in collagen

\begin{tabular}{|l|l|}
\hline Parameter measured & Information obtained or conclusions d rawn \\
\hline Magnitude of SHG signal & $\begin{array}{l}\text { SHG signal magnitude varies greatly with position. } \\
\text { Collagen fibrils are oriented randomly parallel and anti- } \\
\text { parallel to their neighbors. } \\
\text { Thermal denaturation and enzymatic digestion result in } \\
\text { a loss of SHG signal. }\end{array}$ \\
\hline $\begin{array}{l}\text { Ratio } R \text { of the signal at } \\
\text { second modulation } \\
\text { harmonic to signal at first } \\
\text { modulation harmonic }\end{array}$ & $\begin{array}{l}\text { Ratio R provides information about the second order } \\
\text { nonlinear susceptibility of collagen. } \\
\text { Hydration affects the nonlinear susceptibility; increased } \\
\text { nonenzymatic crosslinking of tissue does not have a } \\
\text { significant effect. }\end{array}$ \\
\hline $\begin{array}{l}\text { Phase of the first } \\
\text { modulation harmonic }\end{array}$ & $\begin{array}{l}\text { Phase provides information about collagen fibril } \\
\text { orientation. } \\
\text { Orientation can be measured in transmission mode or in } \\
\text { reflection mode, to a depth of about } 200 \mu \mathrm{m} \text { in tissue. }\end{array}$ \\
\hline
\end{tabular}




\section{FUTURE DIRECTIONS}

In this work, second harmonic generation in collagen has been studied in detail, primarily in a highly organized, much-studied model tissue-rat-tail tendon. These studies have provided insight into the relationship between collagen structure and organization and its second order nonlinear susceptibility. The reason for the strong fluctuation in second harmonic signal as a function of position in highly uniform tissue samples has been determined. The effect of structural modifications in collagen has been studied. The ability of polarization-modulated SHG to measure collagen orientation in tissue has been demonstrated.

A number of practical applications of SHG microscopy to studying collagen have also been demonstrated in this work: imaging of osteoarthritic knee cartilage and fibrotic liver. The potential applications of this technique to studying collagen-both for the purpose of diagnosing disease and for the purpose of understanding fundamental disease processes in the laboratory-are vast. In skin (the organ most accessible to optical microscopy) alone, SHG microscopy could be used to study Ehlers-Danlos syndrome, investigate the rearrangement of the extracellular matrix induced by the spread of melanoma, and examine the scarring produced by burn injury or abnormal wound healing. Endoscope mounted laser fluorescence confocal microscopes currently being designed and built could readily be adapted to SHG imaging-greatly expanding the range of tissues in which this technique could be applied (Watson et. al., 2002; Liang et. al., 2002).

The key hurdle to the application of SHG microscopy to a wider range of problems in the biology and pathology of collagen is a practical one. The experimental 
system used in this work uses a motorized translation stage to scan the sample beneath the laser beam. Image acquisition over a reasonable scan area and at a reasonable resolution $(100 \mu \mathrm{m}$ by $100 \mu \mathrm{m}$ region at $1 \mu \mathrm{m}$ resolution) requires several minutes. The system is mounted directly on an optical table, and time-consuming realignment of the optical components is sometimes required. Commercially available two photon microscopes (the Zeiss LSM 510 or the Radiance 2100 MP from Bio-Rad Laboratories, for example) built around inverted microscopes and designed for confocal microscopy or two photon fluorescence imaging can readily be converted to SHG imaging. Such systems are designed to be extremely stable and typically require realignment only when they are modified significantly. These microscopes also use galvanometer mirrors to scan the laser beam rapidly across the sample, rather than moving the sample stage; given strong enough signal, video-rate image acquisitions times are possible. The disadvantage of these systems is that they typically cost more than $\$ 250,000$ and they are more difficult to modify than an optical table mounted microscope. To justify their purchase, such systems must be accessible to a large group of researchers: thus, they must be stable, reliable, and simple to operate.

The advantages of polarization-modulated SHG microscopy could certainly be incorporated in a user-friendly, inverted, beam-scanning microscope. Construction of this system on an optical table has allowed potential weaknesses of the design to be discovered and resolved. Two major modifications would be required to incorporate this system into an inverted microscope. First, a beam-scanning system is necessary to achieve the high image acquisition rates that would make such a system practical for biological research; such systems are commercially available and can be purchased 
separately or as components of two photon fluorescence microscopy systems. A second issue that must be addressed is the limited bandwidth of the EOM used in the current system. The modulation frequency is limited to $\sim 10 \mathrm{kHz}$ by the EOM and high voltage power supply combination. For high speed imaging, an EOM operating over a bandwidth of $0 \mathrm{MHz}$ to $100 \mathrm{MHz}$ would be required. Although higher modulation frequencies are possible, commercially available Ti:Sapphire ultra-short pulse oscillators operate at repetition rates of $\sim 80 \mathrm{MHz}$, making a faster EOM unnecessary. Phase sensitive detection at these frequencies is possible using RF lock-in amplifiers.

High speed imaging is useful only when the signal strength in a given sample is strong enough. At individual image points in a sample, the number of photons detected must be large enough to prevent shot-noise from limiting the measurement. When shotnoise is limiting, the signal to noise ratio is roughly proportional to the square root of the number of photons. Thus, approximately one hundred photons are necessary to reduce the error due to shot noise to $10 \%$. To achieve $10 \%$ error-based on the signal strengths measured in tendon-a signal collection time of $\sim 1 \mu$ s per image pixel is necessary. This means that 100 pixel by 100 pixel images could be obtained in approximately $10 \mathrm{~ms}$. Even in tissues with signal strengths that are weaker by one to two orders of magnitude, such images could be obtained in $\sim 1 \mathrm{~s}$.

Clearly, the many possible applications of polarization-modulated SHG to the study of collagen suggest that its incorporation into a user-friendly microscope capable of image acquisition at reasonable frame rates $(\sim 1 \mathrm{~Hz})$ would be of benefit to doctors and scientists interested in the complex structure and pathology of collagen. Moreover, due to many advances made in the last several years, a fast, user-friendly instrument could 
readily be constructed using currently available technology. An imaging system could, for instance, be developed to study collagen-related pathological conditions of the skin (an organ which is readily accessible to optical microscopy to a depth of a few hundred microns). 


\section{References}

C. Lian, K. B. Sung, R. R. Richards-Kortum, and M. R. Descour. 2002. Design of a highnumerical aperture miniature microscope objective for an endoscopic fiber confocal reflectance microscope. Applied Optics. 22:4603-4610.

T. F. Watson, M. A. A. Neil, R. Juŝkaitis, R. J. Cook, and T. Wilson. 2002. Video-rate confocal endoscopy. Journal of Microscopy. 207:37-42. 


\section{Acknowledgements}

To be added later. 